\title{
Monte Carlo Simulation of the EXO Gaseous Xenon Time Projection Chamber and Neural Network Analysis
}

\author{
by
}

\author{
François Léonard
}

\author{
A Thesis submitted to \\ the Faculty of Graduate and Postdoctoral Affairs \\ in partial fulfilment of \\ the requirements for the degree of \\ Master of Science
}
Ottawa-Carleton Institute for
Physics

Department of Physics
Carleton University
Ottawa, Ontario, Canada

April 2012

Copyright $(C$

2012 - François Léonard 
Library and Archives

Canada

Published Heritage

Branch

395 Wellington Street

Ottawa ON K1A ON4

Canada
Bibliothèque et

Archives Canada

Direction du

Patrimoine de l'édition

395 , rue Wellington

Ottawa ON K1A ON4

Canada
Your file Votre référence

ISBN: 978-0-494-91547-9

Our file Notre référence

ISBN: $978-0-494-91547-9$
NOTICE:

The author has granted a nonexclusive license allowing Library and Archives Canada to reproduce, publish, archive, preserve, conserve, communicate to the public by telecommunication or on the Internet, loan, distrbute and sell theses worldwide, for commercial or noncommercial purposes, in microform, paper, electronic and/or any other formats.

The author retains copyright ownership and moral rights in this thesis. Neither the thesis nor substantial extracts from it may be printed or otherwise reproduced without the author's permission.
AVIS:

L'auteur a accordé une licence non exclusive permettant à la Bibliothèque et Archives Canada de reproduire, publier, archiver, sauvegarder, conserver, transmettre au public par télécommunication ou par l'Internet, prêter, distribuer et vendre des thèses partout dans le monde, à des fins commerciales ou autres, sur support microforme, papier, électronique et/ou autres formats.

L'auteur conserve la propriété du droit d'auteur et des droits moraux qui protege cette thèse. $\mathrm{Ni}$ la thèse ni des extraits substantiels de celle-ci ne doivent être imprimés ou autrement reproduits sans son autorisation.
In compliance with the Canadian Privacy Act some supporting forms may have been removed from this thesis.

While these forms may be included in the document page count, their removal does not represent any loss of content from the thesis.
Conformément à la loi canadienne sur la protection de la vie privée, quelques formulaires secondaires ont été enlevés de cette thèse.

Bien que ces formulaires aient inclus dans la pagination, il n'y aura aucun contenu manquant. 


\section{Abstract}

Neutrinoless double beta decay has attracted much interest since its observation would reveal the neutrino masses and determine the Majorana nature of the particle. EXO is among the next generation of experiments dedicated to the search for this phenomenon. A part of the collaboration is developing a gas phase time projection chamber prototype to study the performance of this technique for measuring the half-life of neutrinoless double beta decay in ${ }^{136} \mathrm{Xe}$.

A Monte Carlo simulation of this prototype has been developed using the Geant4 toolkit and the Garfield and Maxwell programs to simulate ionizing events in the detector, the production and propagation of the scintillation and electroluminescence signals and their distribution on CsI photocathodes. The simulation was used to study the uniformity of light deposition on the photocathodes, the effect of the natural gamma background radiation on the detector and its response to calibration gamma sources.

Furthermore, data produced with this simulation were analyzed with a neural network algorithm using the multi-layer perceptron class implemented in ROOT. The performance of this algorithm was studied for vertex reconstruction of ionizing events in the detector as well as for classification of tracks for background rejection. 


\section{Acknowledgments}

I would like to acknowledge my supervisor, Dr. Kevin Graham, for allowing me to work on this very interesting project and for his help and guidance. I would also like to express my gratitude to the members of the EXO collaboration at Carleton University, such as Dr. David Sinclair, Etienne Rollin, Christian Ouellet, Matt Bowcock, Vance Strickland, Philippe Gravelle, Alex Coppens, and Christina Hagemann, for their valuable help and support throughout the course of my degree. I also want to thank the members of the EXO-200 collaboration with whom I had the chance to spend some time during my travels to WIPP, such as Jon Davis, Adam Rivas, Michelle Dolinski, Lisa Kaufman, Eric Beauchamp, Attila Dobi, Simon Slutsky, Ryan MacLellan, Yung-Ruey Yen, and many more. You made my shifts very instructive and my free time around Carlsbad much entertaining. Special thanks to my co-workers and friends Katy Hally, Matt Dunford, Nicholi Shiell, Andrew Erlandson, Maria Paula Rozo, James Lacey, and many more. You all contributed in making the last two years and a half much more enjoyable. Finally, and most importantly, thanks to my father, my mother, and my brother, for supporting and encouraging me in pursuing my studies in Physics. 


\section{Table of Contents}

$\begin{array}{lll}\text { Abstract } & \text { ii }\end{array}$

Acknowledgments

Table of Contents $\quad$ iv

List of Tables $\quad$ vii

List of Figures $\quad$ viii

1 Introduction 1

1.1 The Neutrino . . . . . . . . . . . . . . . . . . . 1

1.2 Neutrino Masses . . . . . . . . . . . . . . . . . 3

1.3 Neutrinoless Double Beta Decay . . . . . . . . . . . . . . . 10

1.4 Overview of the Thesis . . . . . . . . . . . . . . 14

2 EXO Experiment 16

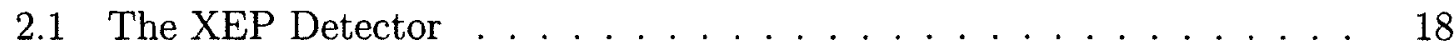

2.2 Detection Process . . . . . . . . . . . . . . . . . . 21

2.2.1 Primary Scintillation and Ionization . . . . . . . 22

2.2.2 Electron Drift and Diffusion ............... 25

2.2.3 Electroluminescence . . . . . . . . . . . . 26

2.3 Experimental requirements . . . . . . . . . . . . . . . . . . 29 
2.3.1 Energy resolution . . . . . . . . . . . . . . 30

2.3 .2 Tracking . . . . . . . . . . . . . . . . . 32

3 XEP Detector Simulation $\quad 34$

3.1 The Geant4 Application . . . . . . . . . . . . . . . 35

3.1 .1 Physics Processes . . . . . . . . . . . . . . . . 35

3.1 .2 Geometry . . . . . . . . . . . . . . 36

3.1 .3 Scintillation . . . . . . . . . . . . . . . 39

3.2 Ionization $\ldots \ldots \ldots \ldots \ldots \ldots \ldots \ldots \ldots \ldots \ldots \ldots$

3.3 Electron Transport with Garfield and Magboltz . . . . . . . . . . 42

3.4 Electroluminescence . . . . . . . . . . . . . . . . . . . . 46

3.5 Examples of Generated Tracks . . . . . . . . . . . . . . 47

4 Simulation Results $\quad 50$

4.1 Detection Process Fluctuations . . . . . . . . . . . . . 50

4.2 Detector Response Uniformity . . . . . . . . . . . . . . . 53

4.3 Natural Gamma Background Simulation . . . . . . . . . . 57

4.3.1 Germanium detector measurement . . . . . . . . . . 58

4.3.2 The Simulation Setup and Results . . . . . . . . . . . 63

4.3.3 Cosmic Ray Background . . . . . . . . . . . . . . 68

4.3.4 Pile-up Estimation . . . . . . . . . . . . . . . . . 68

4.4 Radioactive sources simulation . . . . . . . . . . . . . . . . . . 69

5 Neural Network $\quad 77$

5.1 Multilayer Perceptron . . . . . . . . . . . . . . . 78

5.1 .1 Feedforward propagation . . . . . . . . . . . . . . 79

5.1 .2 Error back-propagation . . . . . . . . . . . . 82

5.1 .3 Weights Adjustment . . . . . . . . . . . . . . . 84 
5.2 ROOT's Multilayer Perceptron Class . . . . . . . . . . . . 86

5.3 The Neural Network Analysis . . . . . . . . . . . . . . . 87

5.4 Number of Track Classification . . . . . . . . . . . . . . . 88

5.5 Multi-Site Background Rejection . . . . . . . . . . . . 94

5.6 Vertex Reconstruction . . . . . . . . . . . . . . . . 96

6 Conclusion 102

$\begin{array}{ll}\text { References } & 105\end{array}$ 


\section{List of Tables}

1.1 Neutrino Oscillation parameters. . . . . . . . . . . . . . . 8

3.1 Material optical properties used in Geant4. . . . . . . . . . . . . . . . 40

3.2 Transport properties of electrons in gaseous Xe calculated with Garfield 45

4.2 Gamma detection rate at various isotopes energies measured in the assembly area with a Ge detector . . . . . . . . . . . . . 61

4.3 Simulation results for the detection rate of gamma rays from ${ }^{40} \mathrm{~K}$ and

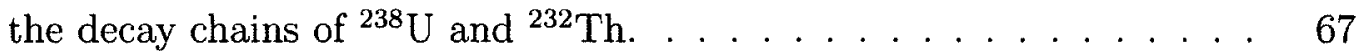

4.4 Simulation results for the detection rate of gamma rays from ${ }^{40} \mathrm{~K}$ and

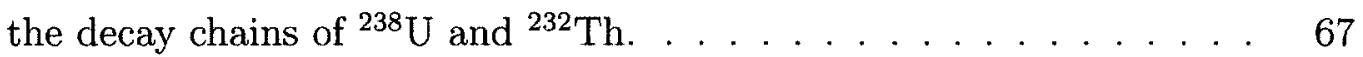

4.5 Photopeak and total detection efficiency for gamma sources located over the center of the Xe active area. . . . . . . . . . . . 73

4.6 Photopeak and total detection efficiency for gamma sources located 24 $\mathrm{cm}$ from the position of the drift field cathode. . . . . . . . . 73

4.7 Maximum gamma source activity for different isotopes . . . . . . . 76

5.1 Fit parameters of the fits from Figures 5.12a and 5.12b. . . . . . . 100

5.2 Fit parameters of the fits from Figures 5.13a and 5.13b. . . . . . . 101 


\section{List of Figures}

1.1 The $p p$-chain of the standard solar model. . . . . . . . . . . . . 4

1.2 Diagram of the two neutrino mass hierarchies f . . . . . . 8

1.3 Feynman diagrams of neutrinoless double beta decay and the regular two-neutrino double beta decay. . . . . . . . . . . . . . . . 11

1.4 Distribution of the total energy of the two electrons emitted in $\beta \beta(2 \nu)$ and $\beta \beta(0 \nu) \ldots \ldots \ldots \ldots \ldots \ldots \ldots$

1.5 Allowed region for the effective neutrino mass, $\left\langle m_{\beta \beta}\right\rangle$, and the smallest neutrino mass $\left(m_{1}\right.$ or $\left.m_{3}\right)$ for the normal and the inverted hierarchy. . 14

2.1 Open side view of the XEP TPC . . . . . . . . . . . . 19

2.2 Illustration of the readout package . . . . . . . . . . . . 20

3.1 Visualization of the detector modelled in Geant4 . . . . . . . . . . . 37

3.2 Illustration of the components of the room where the detector is being built that were included in the simulation. . . . . . . . . . . 38

3.3 Plot from Garfield showing 20 electrons drifting from the same point over $60 \mathrm{~cm}$ in 10 atmosphere xenon at $300 \mathrm{~K}$ under the effect of a 1 $\mathrm{kV} / \mathrm{cm}$ uniform electric field. . . . . . . . . . . . . . . 43

3.4 Example of a simulated $1 \mathrm{MeV}$ electron track . . . . . . . . 48

3.5 Time spectrum of the photon arrival times on the two photocathodes

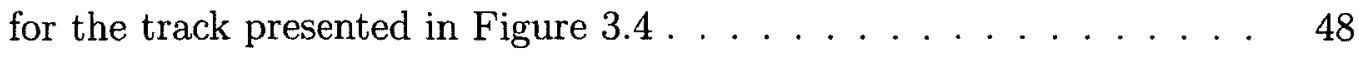

3.6 Example of a simulated $\beta \beta(0 \nu)$ event . . . . . . . . . . . . . . 49 
3.7 Time spectrum of the photon arrival times on the two photocathodes for the track presented in Figure $3.6 \ldots \ldots \ldots \ldots$. . . . . . . .

4.1 Distribution of the energy deposited in Xe, the numbers of scintillation photons, ionization electrons, and EL photons generated,. and the number of EL photons detected on each photocathodes . . . . . . . .

4.2 Distribution on the photocathode on the EL region side of the detected EL photons from 10000 electrons generated at different locations in the $\mathrm{x}$-y plane. . . . . . . . . . . . . . . . .

4.3 Distribution on the photocathode at the far end of the TPC of the detected EL photons from 10000 electrons generated at different locations on the $\mathrm{x}-\mathrm{y}$ plane. $\ldots \ldots \ldots \ldots \ldots \ldots \ldots \ldots$

4.4 Detection efficiency of each photocathode for EL photons originating at various radial positions $\ldots \ldots \ldots \ldots \ldots \ldots$

4.5 Spectrum of the natural gamma ray background measured in the assembly area with a Ge Detector . . . . . . . . . . . . . . . . 59

4.6 Example of fit for the extraction of the number of counts in a photopeak from a ${ }^{137} \mathrm{Cs}$ source $\ldots \ldots \ldots \ldots \ldots \ldots$. . . . . . . . . . 60

4.7 Fit of the measured efficiencies of the Ge detector . . . . . . . . 60

4.8 Simulated spectrum from ${ }^{238} \mathrm{U}$ with $10 \mathrm{~atm} \mathrm{Xe} . \ldots \ldots \ldots$

4.9 Simulated spectrum from ${ }^{232} \mathrm{Th}$ with $10 \mathrm{~atm}$ Xe. . . . . . . . . . 66

4.10 Simulated spectrum from ${ }^{40} \mathrm{~K}$ with $10 \mathrm{~atm} \mathrm{Xe} \ldots \ldots \ldots$

4.11 Position of the simulated gamma sources. . . . . . . . . 70

4.12 Spectra of the energy deposited by the $356 \mathrm{keV}$ gamma rays at two

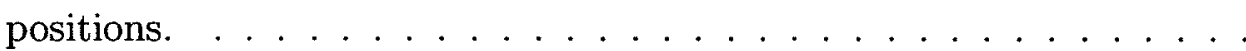

4.13 Spectra of the energy deposited by the $661.7 \mathrm{keV}$ gamma rays at two

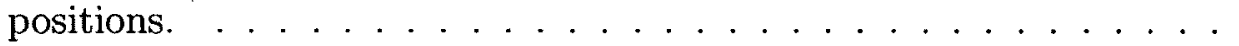


4.14 Spectra of the energy deposited by the $1173.2 \mathrm{keV}$ and $1332.5 \mathrm{keV}$ gamma rays at two positions. . . . . . . . . . . .

4.15 Spectra of the energy deposited by the $511.0 \mathrm{keV}$ and $1274.5 \mathrm{keV}$ gamma rays at two positions. . . . . . . . . . . . .

4.16 Spatial distribution of the energy deposition rate in the active Xe from a $661.7 \mathrm{keV}$ mono-energetic gamma source located on the top of the TPC over the center of the active Xe volume. . . . . . . . 74

5.1 An example of a multilayer perceptron neural network. . . . . . . 79

5.2 Sigmoid and Tanh functions. . . . . . . . . . . . . . . . . . 81

5.3 Distribution of output from a neural network with two hidden layers of 5 neurons each trained with the stochastic minimization algorithm over 50 epochs. . . . . . . . . . . . . . .

5.4 Distribution of output from a neural network with two hidden layers of 10 neurons each trained with the stochastic minimization algorithm

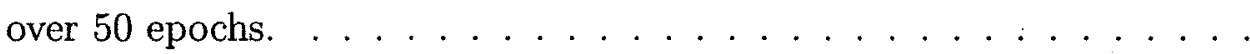

5.5 Distribution of output from a neural network with two hidden layers of 50 neurons each trained with the stochastic minimization algorithm over 50 epochs. . . . . . . . . . . . . . . . . . . . 91

5.6 Distribution of output from a neural network with a single layer of 2 neurons trained with the stochastic minimization algorithm over 50

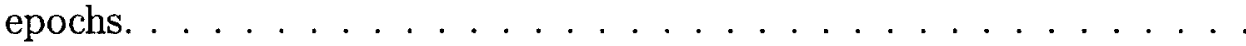

5.7 Distribution of output from a neural network with two layers of 5 neurons each with the BFGS method over 100 epochs. . . . . . . . 93

5.8 Distribution of the output of a neural network with two hidden layers of 6 and 4 neurons trained with the stochastic minimization and 50 epochs. . . . . . . . . . . . . . . . 
5.9 Distribution of the output of a neural network with two hidden layers of 6 and 4 neurons trained with the BFGS method and 100 epochs. .

5.10 Scatter plot of the calculated output as a function of the target value (true value) for a neural network with a single layer of 50 neurons trained over 20 epochs with the stochastic minimization algorithm.

5.11 Scatter plot of the output calculated as a function of the target value for a neural network with two hidden layers of 60 neurons each trained with the stochastic minimization method over 20 epochs. . . . . . . .

5.13 Distribution of the difference between the output and the target values of a neural network with two layers of 60 neurons trained with the stochastic minimization algorithm over 20 epochs. . . . . . . . . . 101 


\section{Chapter 1}

\section{Introduction}

\subsection{The Neutrino}

The existence of the neutrino was first proposed in 1930 by Wolfgang Pauli in order to explain the continuous energy spectrum of the electron emitted in nuclear beta decay [1]. Sensitive measurements of this process revealed an important difference of energy and momentum between the initial nuclei on one side and the final recoiling nuclei and the emitted electron on the other side. To explain this apparent violation of energy and momentum conservation, Pauli theorized the existence of an electrically neutral particle that escaped the experiments undetected, carrying away the missing energy and momentum. In modern terminology, this process can then be written as $n \rightarrow p^{+}+e^{-}+\bar{\nu}_{e}$. In 1932, Enrico Fermi included the particle, which he named neutrino, in his theoretical framework of beta decay [1].

The neutrino was first experimentally detected in 1957 by Clyde Cowan and Frederick Reines [2]. In their experiment, the flux of antineutrinos from the Savannah River nuclear power plant was observed through the inverse beta-reaction $\bar{\nu}_{e}+p^{+} \rightarrow e^{+}+n$. It was already suspected at that time that two distinct 'flavours' of neutrinos existed, each related to a different charged lepton: the electron $(e)$ and the muon $(\mu)$. This distinction was first experimentally established in 1961 by Leon 
Lederman, Melvin Schwartz and Jack Steinberger [3]. In their experiment, which involved the development of the first neutrino beam, neutrinos produced mostly through muon decays were directed toward a spark chamber, where their impact on aluminium plates produced muon trails that could be detected. Subsequently, experiments in the 1970's revealed the existence of a third lepton, the tau $(\tau)$ [4], and it was immediately recognized that a third neutrino flavour should also exist. It is only in 2001 that the DONUT experiment at Fermilab directly measured for the first time the tau neutrino [5].

The standard model of particle physics now counts three generations (flavours) of neutrinos: the electron-neutrino $\left(\nu_{e}\right)$, the muon-neutrino $\left(\nu_{\mu}\right)$ and the tau-neutrino $\left(\nu_{\tau}\right)$. These three particles, together with the electrically charged electron, muon and tau, form the group of particles known as leptons. In addition, each of the six leptons have an antiparticle counterpart, defined as having the same mass but opposite charge and quantum numbers.

One of those quantum numbers is the lepton number, also called the lepton charge. Each lepton is assigned a lepton number of 1 , and each anti-lepton a lepton number of -1 . In any weak interaction, such as the beta decay or the muon decay mentioned above, the total lepton number has been observed to be the same before and after the interaction. This implies that the lepton number is a conserved quantity under weak interactions. Moreover, a similar conservation law exists for each individual lepton flavor with the definition of an electron, a muon, and a tau lepton number. However, the conservation of these individual lepton numbers has been observed to be violated by neutrino oscillation, which is discussed in the next section,

Leptons are part of a larger family of particles called fermions, which groups all the particles that obey Fermi-Dirac statistics. Electrically neutral fermions can be either Dirac or Majorana particles. The main difference between the two is that Dirac fermions are distinct from their antiparticles, while Majorana fermions are identical to 
them. While most fermions are known to be Dirac particles, the nature of neutrinos has not yet been established.

This is primarily because neutrinos are very difficult to observe experimentally. Contrary to the three charged leptons, the three neutrinos are electrically neutral and can only interact with other particles via the weak and the gravitational forces. Hence, they have a very low probability of interaction with other particles. For this reason, many of their properties have not yet been observed, such as their mass and whether they are Dirac or Majorana fermions. Establishing all the properties of neutrinos is one of the goals of particle physics and many experiments have been carried out over the past decades for this purpose.

\subsection{Neutrino Masses}

One of the first important insights into the nature of the neutrino masses came from the study of the burning mechanism of the Sun. After it was understood that the Sun burns through nuclear fusion, it became clear that neutrinos should be emitted from it. Bethe and Critchfield proposed the first model of solar thermonuclear reactions to explain how the Sun produces its energy [6]. In their model, the energy of the Sun is produced through the fusion of two protons to a deuteron, a positron and an electron neutrino: $p^{+}+p^{+} \rightarrow{ }^{2} H^{+}+e^{+}+\nu_{e}$. Today's standard solar model predicts that $98 \%$ of the Sun energy is provided by the $p p$-chain, where four protons are converted to ${ }^{2} \mathrm{He}: 4 p^{+} \rightarrow{ }^{4} \mathrm{He}^{++}+2 e^{+}+2 \nu_{e}[1]$. The complete list of reactions involved in the $p p$-chain is shown in Figure 1.1.

To investigate the solar model, it was proposed to detect neutrinos from the Sun via neutron capture on chlorine with the reaction ${ }^{37} \mathrm{Cl}+\nu_{e} \rightarrow{ }^{37} \mathrm{Ar}++e^{-}$. This idea was first applied in 1960 at the Homestake gold mine in South Dakota in an experiment led by Ray Davis [7]. After the first results were published in the mid 


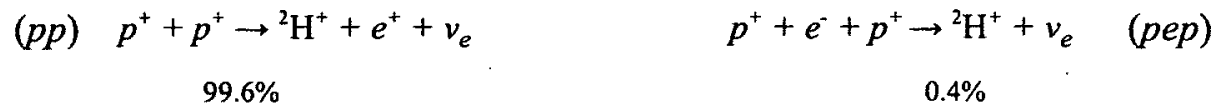

$$
\begin{aligned}
& { }^{2} \mathrm{H}^{+}+p^{+} \stackrel{\bullet}{\rightarrow}{ }^{3} \mathrm{He}^{++}+\gamma
\end{aligned}
$$

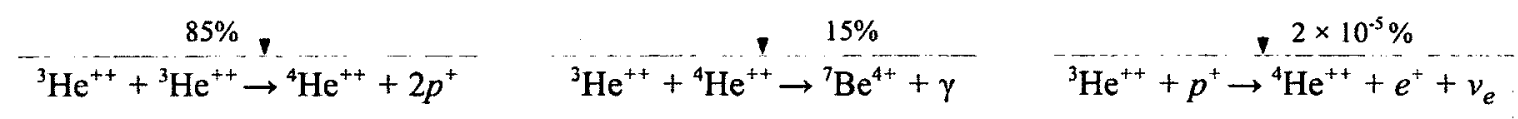

$$
\begin{aligned}
& \text { (hep) }
\end{aligned}
$$

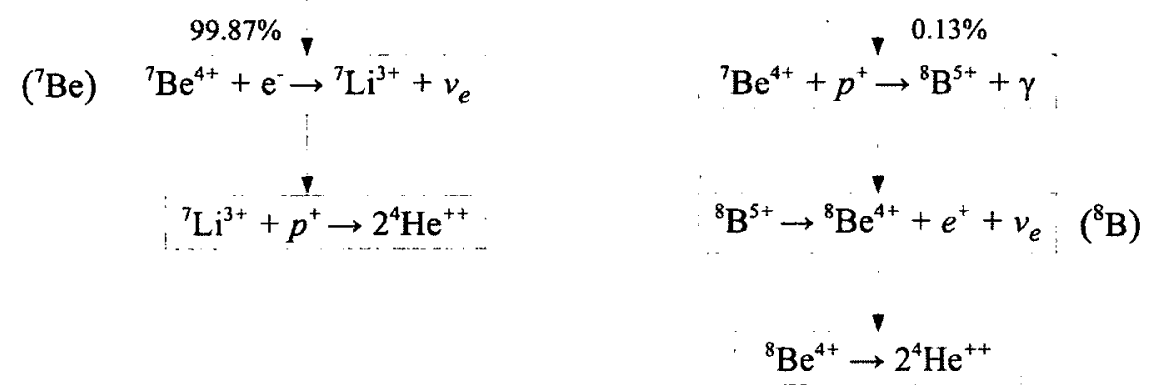

Figure 1.1: The chain of reactions, known as the pp-chain, responsible for the production of $98 \%$ of the Sun's energy, with the branching ratios of each reaction and the nomenclature commonly used for the different solar neutrinos produced.

1960's, a fact became apparent: the measured flux of solar neutrinos was significantly lower than the theoretical models predictions. This neutrino deficiency was later confirmed and reinforced by several other experiments, such as the Kamiokande II [8], the GALLEX [9] and the SAGE [10] experiments. It was shown that no change in the solar model could completely explain the difference between the data and the prediction [1].

A number of hypotheses were proposed to explain the deficit of solar neutrinos in experimental data. One of them was that the flavour of a neutrino might change as it propagates through space-time. This idea was first discussed in 1969 by Bruno Pontecorvo, who postulated that neutrinos might oscillate between the electron and muon flavours, the only two known at the time [11]. This can happen if neutrinos of 
definite flavour $\left(\nu_{l}, l=e, \mu, \tau\right)$ are not state of definite mass, but rather exist as a coherent superposition of mass states $\left(\nu_{i}, i=1,2,3\right)$. This can be expressed as

$$
\nu_{l}=\sum_{i} U_{l i} \nu_{i}
$$

where $U_{l i}$ are the elements of the neutrino mixing matrix. In addition to Pontecorvo, this matrix is often associated to the work of Maki, Nakagawa and Sakata [12] and hence is sometime denoted $U_{M N S}$ or $U_{P M N S}$.

Until then, there had been no evidence that neutrinos had any mass at all, and it was often assumed that they were massless. However, the change of neutrino flavour through oscillation requires neutrinos to be massive. For only two neutrino flavours, the probability of a neutrino with flavour $l$ to be observed with flavour $k$ after having travelled a distance $L$ is given by [1]

$$
P\left(\nu_{l} \rightarrow \nu_{k}\right)=\sin ^{2} 2 \theta \sin ^{2}\left(1.27\left|\Delta m_{j i}^{2}\right| \frac{L}{E_{\nu}}\right)
$$

where $E_{\nu}$ is the neutrino energy in $\mathrm{GeV},\left|\Delta m_{j i}^{2}\right|=\left|m_{j}^{2}-m_{i}^{2}\right|$ is the mass squared difference in $\mathrm{eV}, L$ is in $\mathrm{km}$, and $\theta$ is called the mixing angle. In the two flavours case, this angle is sufficient to parametrize the mixing matrix from Equation 1.1:

$$
\left(\begin{array}{l}
\nu_{e} \\
\nu_{\mu}
\end{array}\right)=\left(\begin{array}{cc}
\cos (\theta) & \sin (\theta) \\
-\sin (\theta) & \cos (\theta)
\end{array}\right)\left(\begin{array}{l}
\nu_{1} \\
\nu_{2}
\end{array}\right) .
$$

In the case of three flavours, the mixing matrix is parametrized by three angles, denoted $\theta_{12}, \theta_{13}$, and $\theta_{23}$, one CP violating phase $\delta$, and two Majorana phases $\alpha_{1}$ and $\alpha_{2}$. Using $c_{i j}$ for $\cos \left(\theta_{i j}\right)$ and $s_{i j}$ for $\sin \left(\theta_{i j}\right)$, the mixing matrix can then be written 


$$
\left(\begin{array}{c}
\nu_{e} \\
\nu_{\mu} \\
\nu_{\tau}
\end{array}\right)=\left(\begin{array}{ccc}
c_{12} c_{13} & s_{12} c_{13} & s_{13} \\
-s_{12} c_{23}-c_{12} s_{23} s_{13} e^{i \delta} & c_{12} c_{23}-s_{12} s_{23} s_{13} e^{i \delta} & s_{23} c_{13} e^{i \delta} \\
s_{12} s_{23}-c_{12} c_{23} s_{13} e^{i \delta} & -c_{12} s_{23}-s_{12} c_{23} s_{13} e^{i \delta} & c_{23} c_{13} e^{i \delta}
\end{array}\right)\left(\begin{array}{c}
e^{i \alpha_{1} / 2} \nu_{1} \\
e^{i \alpha_{2} / 2} \nu_{2} \\
\nu_{3}
\end{array}\right)
$$

The first evidence that supported the neutrino oscillation theory came from the study of atmospheric neutrinos. The Earth is constantly bombarded by high energy radiation which interacts with the atmosphere to produce cascades of secondary particles called hadronic showers. These cascades contain muons and pions which create most of the flux of atmospheric neutrinos through their respective decays:

$$
\begin{array}{ll}
\pi^{+} \rightarrow \mu^{+}+\nu_{\mu}, & \pi^{-} \rightarrow \mu^{-}+\bar{\nu}_{\mu} \\
\mu^{+} \rightarrow e^{+}+\nu_{e}+\bar{\nu}_{\mu}, & \mu^{-} \rightarrow e^{-}+\bar{\nu}_{e}+\nu_{\mu}
\end{array}
$$

Atmospheric neutrinos provide the opportunity to study the oscillation over a wide range of path lengths. Since they interact very weakly with matter, neutrinos produced on one side of the Earth contribute to the flux on the opposite side after travelling through the diameter of the planet. Therefore, the oscillation can be observed from a path length of around $10 \mathrm{~km}$ for downward going neutrinos to roughly $10,000 \mathrm{~km}$ for the upward going ones.

The Super-Kamiokande experiment published in 1998 the first evidence of neutrino oscillation by measuring the intensity of the muon neutrino flux together with its angular distribution [13]. The relation between the zenith-angle of the flux with its intensity appeared to fit well the hypothesis of $\nu_{\mu} \rightarrow \nu_{\tau}$ oscillation. The results of the 
Super-Kamiokande experiment found the mixing angle $\theta_{23}$ to be near maximal and established the value of $\left|\Delta m_{31}^{2}\right| \sim\left|\Delta m_{32}^{2}\right|$, also denoted $\left|\Delta m_{\text {atm }}^{2}\right|$.

Other experiments have also confirmed the theory of neutrino oscillation. The SNO experiment, which was designed to determine separately the flux of electron neutrinos and the flux of neutrinos of other flavour, published in 2001 its inescapable conclusion that solar neutrinos oscillate as well [14].

Two years later, KamLAND provided the first evidence of oscillation of electron antineutrinos from commercial nuclear reactors [15]. The oscillation obtained from this experiment agreed perfectly with those from the solar $\nu_{e}$ experiments. From the combined analysis of these data, it was possible to obtain the value of $\left|\Delta m_{21}^{2}\right|$, including its positive sign, as well as $\theta_{12}$, also denoted $\theta_{\text {sol }}$.

Finally, an upper limit on the last mixing angle, $\theta_{13}$, was established by the CHOOZ [16] and the Palo Verde [17] experiments. Furthermore, the T2K collaboration announced in June 2011 the first possible observation of $\nu_{\mu} \rightarrow \nu_{e}$ oscillation [18]. Their results determined an interval of $0.03(0.04)<\sin ^{2} \theta_{13}<0.28(0.34)$ at $90 \%$ C.L. for a normal (inverted) hierarchy, which will be explained bellow. If confirmed, this result will constitute the first measurement of $\theta_{13}$.

The mixing of massive neutrino flavours has thus been experimentally established through the observation of reactor, atmospheric and solar neutrinos, and the results are in good agreement with the current formalism of neutrino oscillation. At least two of the three mixing angles and the three mass squared differences have been determined reasonably well. The present known values of the oscillation parameters are summarized in Table 1.1.

With the current knowledge of the neutrino masses accumulated from the oscillation experiments, two possible scenarios can be considered. If the sign of $\left|\Delta m_{31}^{2}\right|$ and $\left|\Delta m_{32}^{2}\right|$ happen to be positive, the pattern of the neutrino masses $\left(m_{1}<m_{2} \ll m_{3}\right)$ 


\begin{tabular}{ccc} 
Parameter & Value & Reference \\
\hline $\sin ^{2} 2 \theta_{12} \equiv \sin ^{2} 2 \theta_{\text {sol }}$ & $0.84_{-0.04}^{+0.03}(68 \%$ C.L. $)$ & {$[19]$} \\
$\sin ^{2} 2 \theta_{23} \equiv \sin ^{2} 2 \theta_{\text {atm }}$ & $>0.92(90 \%$ C.L. $)$ & {$[20]$} \\
$\sin ^{2} 2 \theta_{13}$ & $<0.19(90 \%$ C.L. $)$ & {$[21]$} \\
$\Delta m_{21}^{2} \equiv \Delta m_{\text {sol }}^{2}$ & $8.0_{-0.3}^{+0.4} \times 10^{-5} \mathrm{eV}^{2}(68 \%$ C.L. $)$ & {$[19]$} \\
$\left|\Delta m_{31}^{2}\right| \sim\left|\Delta m_{32}^{2}\right| \equiv\left|\Delta m_{\text {atm }}^{2}\right|$ & $2.4_{-0.5}^{+0.6} \times 10^{-3} \mathrm{eV}^{2}(90 \%$ C.L. $)$ & {$[22]$}
\end{tabular}

Table 1.1: Neutrino Oscillation parameters.

is called "normal hierarchy". On the other hand, if the signs are negative, the pattern $\left(m_{3} \ll m_{1}<m_{2}\right)$ is then called "inverted hierarchy". The two hierarchies are illustrated in Figure 1.2.

In addition to neutrino oscillation, attempts have been made to study the neutrino masses through the kinematics of the charged particles (leptons and pions) emitted in various weak decays. The most sensitive techniques probe the mass of the electron

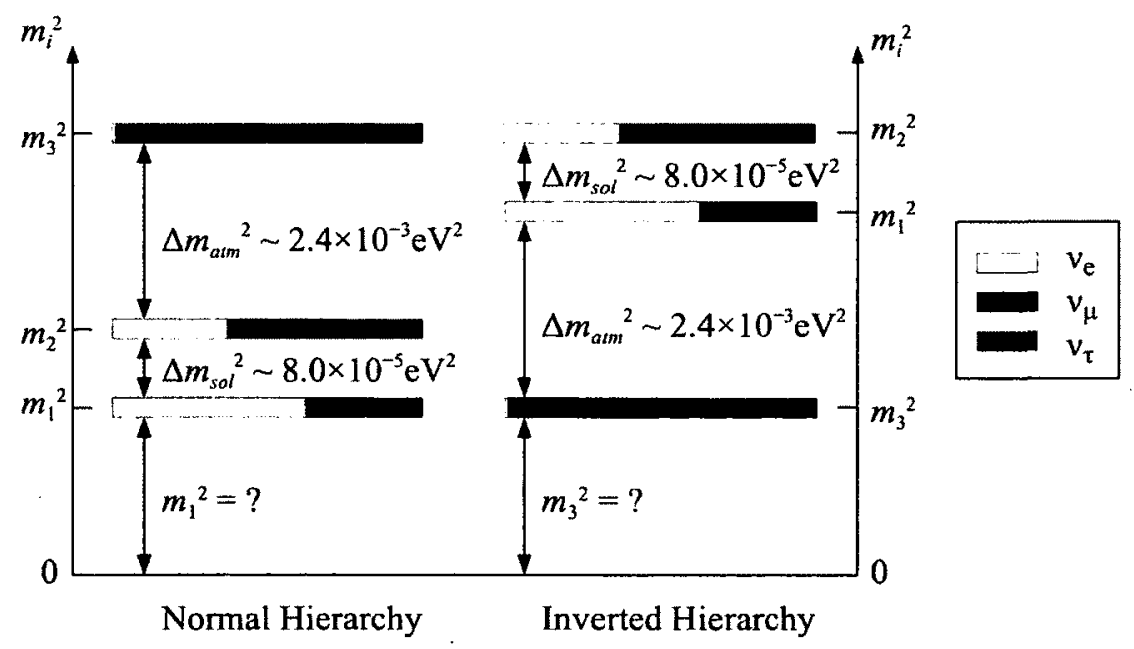

Figure 1.2: Diagram of the two neutrino mass hierarchies. 
neutrino, defined as

$$
m_{\nu_{e}}=\sqrt{\sum_{i}\left|U_{e i}\right|^{2} m_{\nu i}^{2}}
$$

by fitting the shape of the beta decay spectrum. Tritium is often used in such experiments. The best limit to date $\left(m_{\nu_{e}}<2.3 \mathrm{eV}\right.$ at $95 \%$ C.L. $)$ was established by the Mainz and Troitsk experiment [23].

More information about the masses of the neutrinos come from cosmology. It can be shown that the sum of the neutrino masses is related to the mass density of the universe through the equation

$$
\sum_{i} m_{\nu_{i}}=92.5 \Omega_{m} h^{2} \mathrm{eV}
$$

where $\Omega_{m}$ is the mass contribution to the cosmological constant and $h$ is the Planck's constant [24]. Through this relation, cosmological observations have allowed to calculate a model dependent constraint on the sum of neutrino masses of approximately $\sum_{i} m_{\nu_{i}} \leq 0.7 \mathrm{eV}[25]$

Neutrino oscillation, direct measurements of decay kinematics, and cosmology have all allowed to investigate the mass states of the neutrinos. However, neutrino oscillation experiments permit only to probe the squared mass differences $\left(\Delta m_{i j}^{2}\right)$ rather than the masses themselves, while direct measurements and cosmology only provided constraints to the neutrino masses. Furthermore, none of these experiments allow to distinguish between the Dirac and Majorana nature of neutrinos. A possible experimental approach that could bring answers to these questions is the observation of neutrinoless double beta decay. Meanwhile, a more detailed discussion about neutrino masses or the phenomena of neutrino oscillation can be found in K. M. Heeger (2004) [1] and S. F. King (2007) [26]. 


\subsection{Neutrinoless Double Beta Decay}

Neutrinoless double beta decay $(\beta \beta(0 \nu))$ is a nuclear transition where an initial nucleus $(Z, A)$, where $Z$ is the number of protons and $A$ is the nucleon number, decays to a final nucleus $(Z+2, A)$, emitting two electrons in the process:

$$
(Z, A) \rightarrow(Z+2, A)+2 e^{-}
$$

This process, illustrated in Figure 1.3a, is driven by the exchange of a light Majorana neutrino and violates the conservation of lepton number. It can only occur if neutrinos have masses (which as been confirmed by the observation of neutrino oscillation) and if they are Majorana particles. Furthermore, it is very similar to another process called two-neutrino double beta decay $(\beta \beta(2 \nu))$, in which two electron antineutrinos are emitted along with the two electrons:

$$
(Z, A) \rightarrow(Z+2, A)+2 e^{-}+2 \nu_{e}
$$

This decay, illustrated in Figure 1.3b, does not violate lepton number conservation and occurs whether or not neutrinos are their own antiparticles. Both types of double beta decay can only be observed in a limited number of isotopes where the initial nucleus $(\mathrm{Z}, \mathrm{A})$ is less bound than the final one $(\mathrm{Z}+2, \mathrm{~A})$, but more than the intermediate one $(\mathrm{Z}+1, \mathrm{~A})$, such that the regular one-electron beta decay is forbidden.

Two-neutrino double beta decay was first proposed in 1935 by Maria GoeppertMayer, who derived an expression for the decay rate and estimated a half-life of about $10^{17}$ years [27]. It was two years later that Ettore Majorana formulated his theory of neutrinos in which the particle is its own antiparticle [28], and Giulio Racah was the first to suggest testing Majorana's theory with neutrinoless double beta decay [29]. 


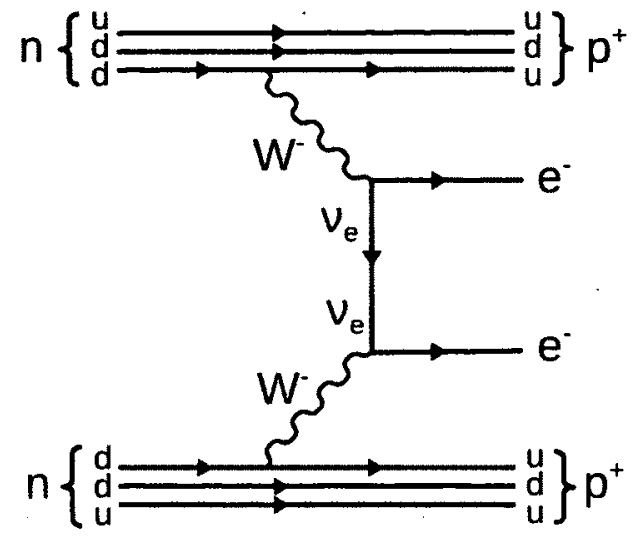

(a)

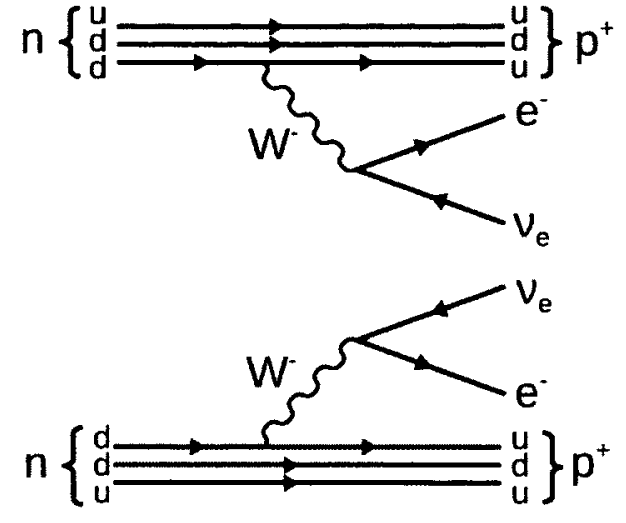

(b)

Figure 1.3: Feynman diagrams of neutrinoless double beta decay (a) and the regular two-neutrino double beta decay (b).

In 1939, Wolfgang Furry calculated the approximate value of the rate of $\beta \beta(0 \nu)$ [30], and in 1952, Primakoff calculated the electron energy spectra and the angular correlation for both $\beta \beta(2 \nu)$ and $\beta \beta(0 \nu)$ [31]. This provided a potentially useful tool for distinguishing between the two processes in experiments.

$\beta \beta(2 \nu)$ has already been observed for a number of isotopes in various experiments. On the other side, except for one unconfirmed claim from Klapdor-Kleingrothaus et al. [32], $\beta \beta(0 \nu)$ has never been seen and a number of experiments are currently ongoing around the world to search for it. An observation of this decay would automatically establish the Majorana nature of the neutrinos, and a measurement of its decay rate would provide knowledge about all three mass eigenstates. The decay rate for $\beta \beta(2 \nu)$ can be written as [33]

$$
\left(T_{1 / 2}^{2 \nu}\right)^{-1}=G_{2 \nu}\left(Q_{\beta \beta}, Z\right)\left|M_{2 \nu}\right|^{2}
$$

where $G_{2 \nu}\left(Q_{\beta \beta}, Z\right)$ is the phase space factor for the emission of the four particles and $M_{2 \nu}$ is the nuclear matrix element. The decay rate of $\beta \beta(0 \nu)$, when mediated by the exchange of a light massive neutrino, is given by [33] 


$$
\left(T_{1 / 2}^{0 \nu}\right)^{-1}=G_{0 \nu}\left(Q_{\beta \beta}, Z\right)\left|M_{0 \nu}\right|^{2}\left\langle m_{\beta \beta}\right\rangle^{2}
$$

where $G_{0 \nu}\left(Q_{\beta \beta}, Z\right)$ is the phase space factor for the emission of the two electrons, $M_{0 \nu}$ is another nuclear matrix element, and $\left\langle m_{\beta \beta}\right\rangle$ is the effective Majorana mass of the electron neutrino, defined as

$$
\left\langle m_{\beta \beta}\right\rangle=\left|\sum_{k} m_{k} U_{e k}^{2}\right| .
$$

In this equation, the $m_{k}$ 's are the mass of the three neutrino mass states, and $U_{e k}$ are the mixing matrix elements.

This makes the observation of $\beta \beta(0 \nu)$ one of the best methods to obtain the neutrino masses and the only practical way to establish the Majorana nature of neutrinos. Some experiments have already attempted to detect this decay. One of them is the Heidelberg-Moscow Collaboration in which a subset of the collaborators claims to have observed the peak of $\beta \beta(0 \nu)$ in the energy spectrum of their ${ }^{76} \mathrm{Ge}$ experiment. They reported a half-live of $T_{1 / 2}^{0 \nu}=\left(2.23_{-0.31}^{+0.44}\right) \times 10^{25}$ yr from which they calculated an effective neutrino mass of $\left\langle m_{\beta \beta}\right\rangle=\left(0.32_{-0.03}^{+0.03}\right) \mathrm{eV}$ [32]. The publication of this group received a number of critical replies and the results haven't been confirmed. However, whether or not their claim is valid, this experiment remains to date the most sensitive and allows to establish the best constraint on the effective electron neutrino mass in ${ }^{76} \mathrm{Ge}$.

The very low decay rate of $\beta \beta(0 \nu)$ poses a significant difficulty for its experimental observation. To meet the high sensitivity required for such a rare decay, an extremely stringent background rejection is required, particularly in the energy region of the Q-value, the total energy of the two emitted electrons. In addition to natural radiation background and radioactive contaminants, the measurements can 
also be contaminated by $\beta \beta(2 \nu)$ events. In $\beta \beta(0 \nu)$, the whole energy is transferred to the two emitted electrons, whose energy spectrum should appear as a narrow peak around the Q-value as illustrated in Figure 1.4. In $\beta \beta(2 \nu)$, part of the energy is also transferred to the two neutrinos which escape the experiment undetected, leaving the two electrons with a broad energy distribution. For this reason, a high energy resolution is required to avoid 2-neutrino events to be detected in the Q-value region of interest.

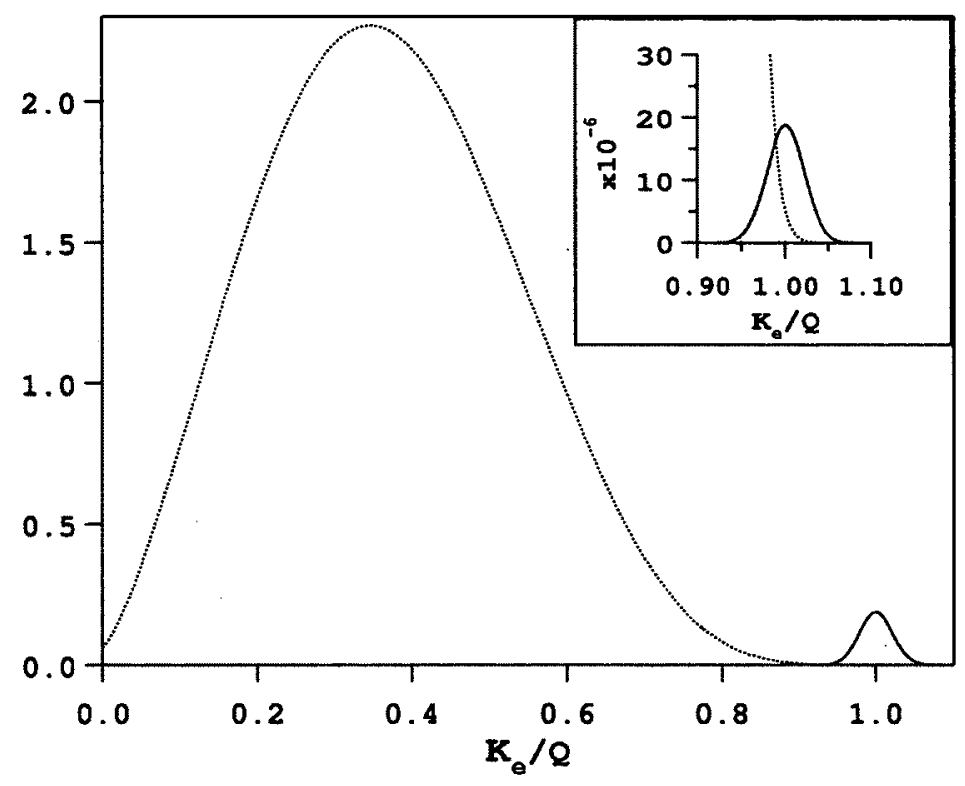

Figure 1.4: Spectra of the total kinetic energy, $\mathrm{K}_{e}$, of the two electrons emitted in $\beta \beta(2 \nu)$ (dotted curve) and $\beta \beta(0 \nu)$ (solid curve), assuming an energy resolution of $5 \%$. The $\beta \beta(2 \nu)$ curve is normalized to one while the $\beta \beta(0 \nu)$ curve is normalized to $10^{-2} \cdot\left(10^{-6}\right.$ in the figure inset). Figure from Reference [34].

With the data from neutrino oscillation and cosmology, it is possible to constrain the effective neutrino mass $\left\langle m_{\beta \beta}\right\rangle$ and the lightest neutrino mass (either $m_{1}$ or $m_{3}$ ). Figure 1.5 shows the dependence of $\left\langle m_{\beta \beta}\right\rangle$ on the lightest mass assuming $\theta_{13}=0$ and using the $\Delta m^{2}$ 's from Table 1.1. As one can see, even if $\left\langle m_{\beta \beta}\right\rangle$ is not measured exactly, determining a more stringent upper bound on its value would allow to distinguish 


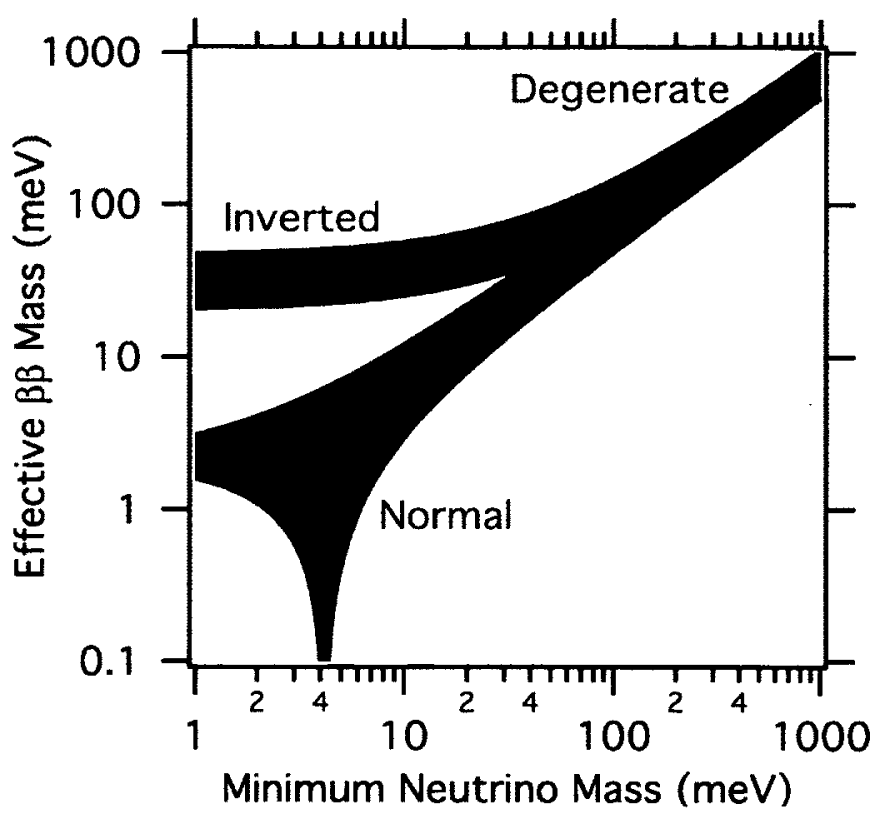

Figure 1.5: Allowed region for the effective neutrino mass, $\left\langle m_{\beta \beta}\right\rangle$, and the smallest neutrino mass $m_{1}$ or $m_{3}$ for the normal and the inverted hierarchy. The plot was made assuming $\theta_{13}=0$ and using the $\Delta m^{2}$ 's from Table 1.1. The filled areas are due to the possible values of the Majorana phases. Figure from Reference [33].

between the normal hierarchy, the inverted hierarchy or the quasidegenerate region.

It should be noted that other mechanisms for neutrinoless double beta decay have been postulated which require the existence of new particles. The one presented above, which occurs through the exchange of a light Majorana neutrino, can be seen as the "minimum" mechanism and it is the most commonly considered. More detailed reviews of neutrinoless beta decay can be found in Avignone et. al. (2008) [33], Elliott and Vogel (2002) [34], or Šimkovic (2011) [35].

\subsection{Overview of the Thesis}

The Enriched Xenon Observatory (EXO) is an experiment designed to search for neutrinoless double beta decay in the 136 isotope of xenon. Carleton University is one of the collaborating institutions and part of its effort focuses on the development 
of a gaseous xenon detector, named Xenon Electroluminescence Prototype (XEP), for the study of a new detection technique. This thesis presents my contribution to the project, which consisted mainly in the development of a Monte Carlo simulation tool of the XEP detector and the study of the performance of a neural network algorithm for event classification and reconstruction.

Before presenting my work, the next chapter introduces the EXO experiment and describes the design and the functioning of the XEP detector, including an explanation of the detection process and the main physical phenomena involved in it. A discussion of the experimental requirements aimed for the detector is also included.

Chapter 3 introduces the Monte Carlo simulation of the XEP detector that I developed using the Geant4 [36] and the Magboltz [37] and Garfield [38] programs. This work included the writing of the Geant4 application and the creation of an interface between Geant 4 and Garfield for the simulation of electron drift. The code used for the generation of neutrinoless and two-neutrino double beta decay events was adapted from the EXO-200 experiment Geant4 simulation.

Chapter 4 presents the results of various studies I performed with the simulation, including an examination of the light collection uniformity in the detector, a study of the effect of the natural background radiation on the detector's performance and an investigation of the detector response to calibration gamma sources. Prior to these studies, I performed measurements of the natural gamma background radiation in the room where the detector is being built with a germanium detector. This study is described in Section 4.3.1.

Finally, Chapter 5 presents the work I did in studying the performances of a multilayer perceptron neural network algorithm to analyze tracks generated with the Monte Carlo simulation. The study was performed using the multi-layer perceptron class implemented in the ROOT software [39]. The performances of this algorithm was examined for background rejection and track vertex reconstruction. 


\section{Chapter 2}

\section{EXO Experiment}

The Enriched Xenon Observatory (EXO) is an experiment led by an international collaboration aiming at developing a ton scale detector for the observation of neutrinoless double beta decay in ${ }^{136} \mathrm{Xe}$. The project currently consists of two prototypes developed to study techniques for working with xenon in a time projection chamber (TPC) in order to measure the half-life of both $\beta \beta(2 \nu)$ and $\beta \beta(0 \nu)$. The first one, EXO-200, is located at the Waste Isolation Pilot Plant (WIPP) in New Mexico and uses approximately $200 \mathrm{~kg}$ of liquid xenon enriched to about $80 \%$ of ${ }^{136} \mathrm{Xe}$. It has been collecting data since spring 2011 and made the first measurement of the halflife of $\beta \beta(2 \nu)$ in this isotope $\left(T_{1 / 2}=2.11 \pm 0.04\right.$ (stat.) \pm 0.21 (sys.) $\left.\times 10^{21} \mathrm{y}\right)[40]$. This was consistent with the result of the KamLAND-Zen collaboration published soon after $\left(T_{1 / 2}=2.38 \pm 0.02\right.$ (stat.) \pm 0.14 (sys.) $\times 10^{21}$ y) [41]. The other prototype, called XEP (Xenon Electroluminescence Prototype), consists of a high pressure xenon gas (HPXe) TPC which is currently being built at Carleton University. It is on this second initiative that the subject of this thesis is based.

Xenon has nine stable isotopes as well as over 40 unstable ones. Among the stable isotopes, four are predicted to undergo double beta decay: ${ }^{124} \mathrm{Xe},{ }^{126} \mathrm{Xe},{ }^{134} \mathrm{Xe}$, and ${ }^{136} \mathrm{Xe}$. However, since this decay possesses a very long half life and has never been observed before, these isotopes are usually considered stable. 
Xenon offers some important advantages over other double beta decay candidate elements. As a noble gas, it is relatively easy to purify in order to remove radioactive or electronegative impurities that could add to the background or degrade the signal. Moreover, xenon is an efficient scintillating material. As will be discussed bellow, the scintillation of xenon plays an essential role in the detection process of the detector.

The 136-isotope of xenon also offers some advantages over other isotopes. First of all, it has a relatively high natural abundance $(\sim 8.9 \%)$ and can be enriched using centrifuges which allow to process large quantities. Furthermore, the Q-value of neutrinoless double beta decay in ${ }^{136} \mathrm{Xe}$ (the total energy of the two electrons emitted) is $2.48 \mathrm{MeV}$, which is above the radioactive uranium decay chain energies. This should reduce the requirement for background suppression. Finally, using ${ }^{136} \mathrm{Xe}$ may allows to completely eliminate the background through barium tagging, a technique to detect the presence of the ${ }^{136} \mathrm{Ba}^{++}$daughter of the decay. If it could be achieved, it could confirm the $\beta \beta(0 \nu)$ origin of a given signal by eliminating all events which do not result in the production of a barium ion. How to practically achieve this is currently being researched at Carleton University as a separate project, but if a barium tagging technique is successfully developed, it could be integrated to the XEP detector in the future.

The next sections are intended to provide an overview of the XEP detector and explain how it functions. The main physical phenomena involved in the detection process of the apparatus are explained in Section 2.2 and the experimental requirements in terms of energy and tracking resolutions that are aimed are presented in Section 2.3 . 


\subsection{The XEP Detector}

The detector is a gas-phase TPC, which is one of the standard devices used in particle physics for the tracking and identification of particles. It consists of a large volume of gas subjected to a constant and uniform electric field. Once a charged particle passes into the volume, it transfers its energy to the gas which is ultimately converted to scintillation photons and ionization electrons. The collection of these photons and electrons form the basis of the measurement of the incident particle's energy and the reconstruction of its track.

The ionization electrons drift under the effect of the electric field toward the anode where the readout plane is located. Due to the collisions with the gas, the electron's drift velocity is constant for a given gas mixture and uniform electric field. The position where the electron is detected on the readout plane gives the $\mathrm{x}$ and $\mathrm{y}$ coordinates of its origin, while the $\mathrm{z}$ coordinate is obtained from the measurement of the drift time, using the primary scintillation to mark the starting time of the drift. Hence, knowing the origin of each ionization electrons, the track of the incident particle can be reconstructed in three dimensions.

In a TPC, the readout planes often consist of multi-wire proportional chambers (MWPC) planes located at both ends of the chamber. However, in the XEP detector, the amplification and detection of the ionization electrons will rather be performed through a technique that exploits electroluminescence (EL). EL is the process of production of secondary scintillation photons occurring when an electron is accelerated with an electric field above the medium's excitation energy. This process can be exploited to convert the ionization signal to a large number of UV photons that will be detected by the same photodetectors as the primary scintillation. As will be explained in Section 2.2.3, EL offer a high gain, low noise and low fluctuation process to measure the energy deposited through ionization and should allow to achieve the 
high energy resolution required for the detection of $\beta \beta(0 \nu)$ [42].

In the design of the XEP TPC, illustrated in Figure 2.1, the xenon gas is contained in a cylindrical stainless steel vessel with an inner diameter of $60 \mathrm{~cm}$ and a total length of about 2 meters. Inside, 28 copper rings $52.2 \mathrm{~cm}$ in diameter will be used to shape the drift electric field with resistors linking them in order to distribute the electric potential across them as a voltage divider. Furthermore, the cage will have at both ends a wire grid serving as anode and cathode. A Teflon cylinder surrounding most of the ring cage will serve as an insulator for the high voltage difference between the rings and the stainless steel vessel as well as an UV light reflector to increase the detection efficiency of scintillation photons.

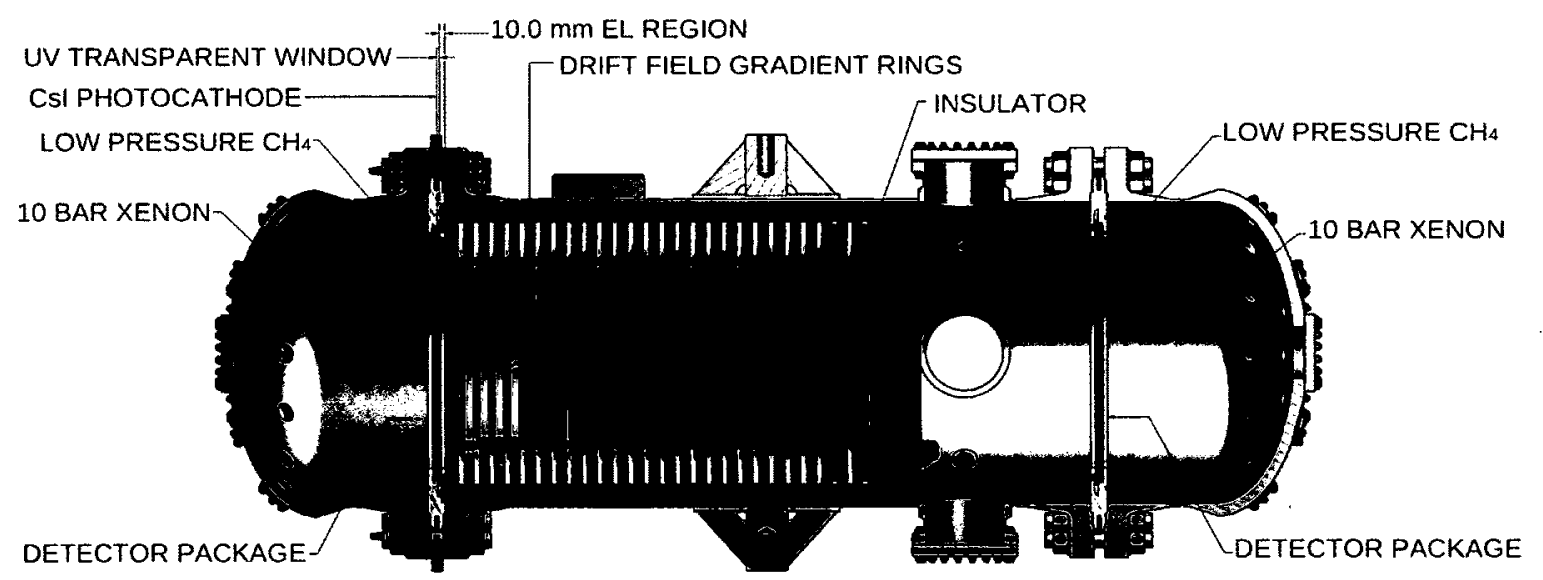

Figure 2.1: Open side view of the XEP TPC.

At both ends of the TPC will be located a cesium iodide (CsI) photocathode for the detection of the primary scintillation and the electroluminescence. The two readout packages, showed in Figure 2.2, will consist of a $6.35 \mathrm{~mm}$ thick fused silica $\left(\mathrm{SiO}_{2}\right)$ window, a $3.17 \mathrm{~mm}$ thick low pressure methane $\left(\mathrm{CH}_{4}\right)$ gas gap, a $\mathrm{CsI}$ layer deposited on a glass fibre circuit board and a Teflon grid between the circuit board and the $\mathrm{SiO}_{2}$ window. Fused silica is known to have a good transparency for UV photons at the wavelength of xenon scintillation $(\sim 175 \mathrm{~nm})$ and therefore is the material of 

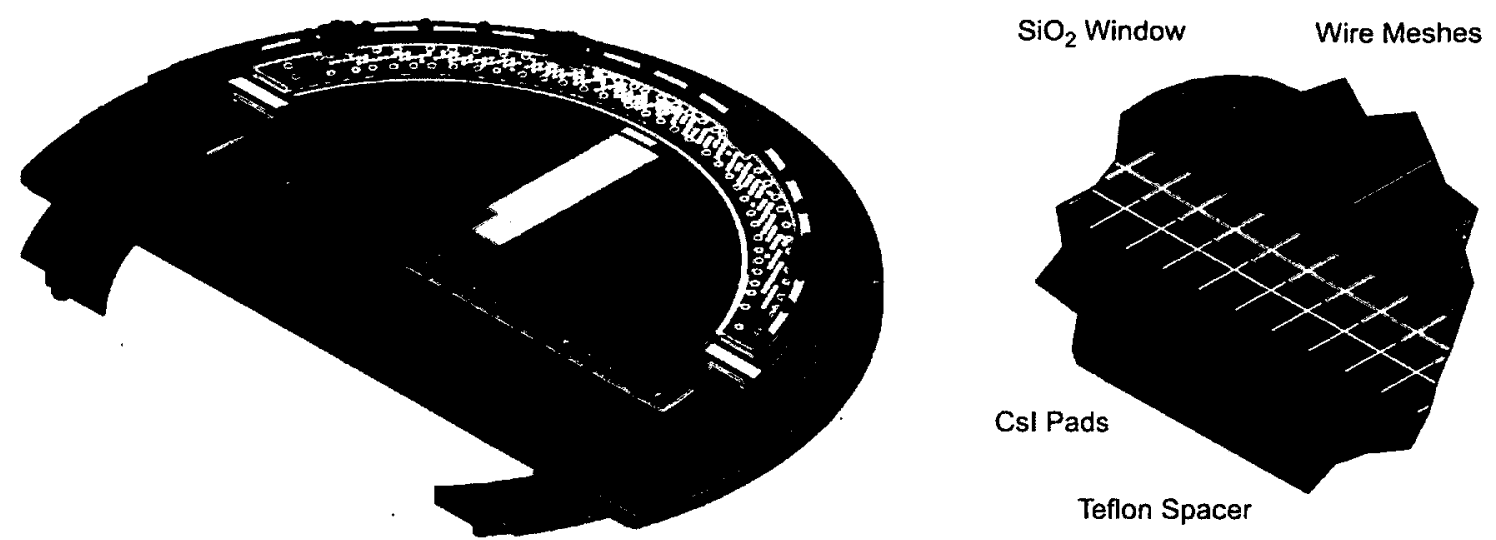

Figure 2.2: Illustration of the readout package, showing the $2.54 \mathrm{~cm} \times 2.54 \mathrm{~cm}$ CsI pads, the Teflon spacer grid within the $\mathrm{CH}_{4}$ gap, the fused silica window and some of the wires that form the electrode meshes of the EL region.

choice to separate the $\mathrm{Xe}$ from the $\mathrm{CH}_{4}$. $\mathrm{CsI}$ is a photo-sensitive material often used in photocathodes for UV photon imaging. It has been shown to provide quantum efficiencies (QE) of approximately 20\% around the wavelength of interest [43]. The thin CsI layer of each readout plane will be segmented in 228 square pads $2.54 \mathrm{~cm}$ on a side, although the openings of the Teflon spacer grid are only $2.24 \mathrm{~cm}$ on a side. When a photon hits one of the pads, it will eject an electron through the photoelectric effect. An electric field in the $\mathrm{CH}_{4}$ gap, between the CsI layer and a conductive wire grid near the $\mathrm{SiO}_{2}$ window, will drift the ejected electrons away from the pad, inducing a signal on it. The role of the Teflon grid in the $\mathrm{CH}_{4}$ gap will be to hold apart the circuit board and the $\mathrm{SiO}_{2}$ window against the very high pressure of the xenon pushing on both sides of the readout packages.

In front of one of the readout plane windows, two wire grids will be located $1 \mathrm{~cm}$ apart from each other to form the EL region, where the EL light from the arriving ionization electrons will be produced. The readout package adjacent to this high field region will record an EL signal which should follow the trajectory of the track and will be used for tracking. On the opposite side of the detector, the EL light should reach the second readout plane quite uniformly, with a soft cosine-like dependence on 
the position, since the photons are emitted isotropically.

The operating pressure of the xenon gas that would provide the optimum performances is unknown at this time. An advantage of a higher pressure is that it allows the same amount of xenon to be contained in a smaller detector, thus reducing the requirement for shielding and potentially providing a lower contamination from external backgrounds. However, the vessel containing this pressure then needs to be made thicker and the background from radioactive impurities inside the vessel becomes more important. It was decided to use a vessel capable of containing a maximum pressure of 10 bars. This compromise was chosen because a pressure vessel capable to withstand vacuum can typically resist to an internal pressure of 10 bars. Therefore, the effect of pressure on the performance of the detector will be studied within a range of 1 to 10 bars.

Once its construction is completed, this detector will allow us to study every aspect of the detection of ionizing events in HPXe and to evaluate the performance of this technology for the search for neutrinoless double beta decay.

\subsection{Detection Process}

As mentioned in the previous section, the primary scintillation will serve as a trigger to indicate the start of the ionizing event, while the ionization of the gas will be used to measure the energy deposited by the incident particle through the production and detection of electroluminescence. Therefore, the main physical processes responsible for the creation of the signal will be the production of scintillation photons and ionization electrons by the incident particle, the drifting of the electrons, and the production of electroluminescence photons. The current understanding of these processes is discussed in the following subsections. 


\subsubsection{Primary Scintillation and Ionization}

When a charged particle with low energy pass through a gas, it loses its energy primarily by ionization and excitation of the gas molecules. According to Platzman [44], when all the kinetic energy of the incident particle is deposited in the gas, the balance of this kinetic energy is given by

$$
E_{C}=N_{i} E_{i}+N_{e x} E_{e x}+N_{i} \epsilon_{h}
$$

where $E_{C}$ is the kinetic energy of the incident particle, $N_{i}$ is the average number of electrons produced by the incident particle, $E_{i}$ is the average potential required to ionize an atom of the gas, $N_{e x}$ is the average number of atoms excited by the incident particle, $E_{e x}$ is the average excitation potential required to excite an atom of the gas, and $\epsilon_{h}$ is the average energy of sub-excitation electrons.

The efficiency of ionization of a gas by a charged particle can be measured by the average energy expended per electron liberated, given by

$$
W_{i}=\frac{E_{C}}{N_{i}}
$$

Similarly, the average energy expended per excited atom is given by

$$
W_{e x}=\frac{E_{C}}{N_{e x}}
$$

$W_{i}$ can be directly obtained by measuring the number of ion pairs produced by ionizing particles. On the other hand, $W_{e x}$ cannot be observed directly. What can be observed is the number of scintillation photons produced by the primary particle $N_{s}$. This 
allows to calculate the average energy expended by the scintillation photons, given by

$$
W_{s}=\frac{E_{C}}{N_{s}}
$$

This value has been measured as $72 \mathrm{eV}$ [45].

$W_{s}$ and $W_{e x}$ are not necessarily equal because, for pressures of more than $10^{3}$ Torr, the scintillation light is produced in two different ways. The first one, called excitation luminescence, occurs through the de-excitation of an excited xenon molecule $X e_{2}^{*}$ as follows [46]:

$$
\begin{aligned}
X e^{*}+X e+X e & \rightarrow X e_{2}^{*}+X e \\
X e_{2}^{*} & \rightarrow X e+X e+h \nu
\end{aligned}
$$

where $X e^{*}$ is an excited xenon atom, $X e$ is a xenon atom, and $h \nu$ is a scintillation photon.

The second origin of scintillation light, called recombination luminescence, involves the recombination of thermalized electrons $e_{t h}$ with atomic xenon ions $X e^{+}$through the following chain of reactions [46]:

$$
\begin{aligned}
X e^{+}+X e+X e & \rightarrow X e_{2}^{+}+X e \\
e_{h o t} & \rightarrow e_{t h}+\text { heat }\left(\epsilon_{h}\right), \\
X e_{2}^{+}+e_{t h} & \rightarrow X e^{* *}+X e \\
X e^{* *} & \rightarrow X e^{*}+\text { heat } \\
X e^{*}+X e+X e & \rightarrow X e_{2}^{*}+X e \\
X e_{2}^{*} & \rightarrow X e+X e+h \nu,
\end{aligned}
$$


where $X e_{2}^{+}$is a molecular xenon ion, $e_{\text {hot }}$ is a sub-excitation electron, $e_{t h}$ is a thermalized electron, and $X e^{* *}$ is a highly excited xenon atom.

Both excitation and recombination luminescence results in the production of photons with a wavelength of $175 \mathrm{~nm}$ and a full width at half maximum (FWHM) of $15 \mathrm{~nm}[46]$. However, an important difference between the two is that recombination luminescence has a dependence on the electric field, due to the coulomb attraction of the ion and the electron in Equation 2.9. If a sufficiently high electric field is applied, the coulomb force can be overcome and the recombination is prevented. In this case, the number of scintillation photons produced, $N_{s}$, is equal to the number of excited atoms, $N_{e x}$, since each excited atom emits one scintillation photon [46].

The time evolution of excitation luminescence is mainly determined by the spontaneous decay of $X e_{2}^{*}$ in Equation 2.6 from its two lowest vibrationally relaxed $(\nu=0)$ excited molecular states $\left({ }^{1} \Sigma_{u}^{+}\right.$and $\left.{ }^{3} \Sigma_{u}^{+}\right)$to its repulsive ground state $\left({ }^{1} \Sigma_{g}^{+}\right)[47]$. Since those two transitions have different decay rates, excitation luminescence has two components, a fast and a slow one. The lifetime of the ${ }^{1} \Sigma_{u}^{+}$singlet state is $4.6 \mathrm{~ns}$, while the lifetime of the ${ }^{3} \Sigma_{u}^{+}$triplet state is $99 \mathrm{~ns}$ [48]. These two transitions are responsible for the primary scintillation light that should be observed in the XEP detector when a charged particle passes through its xenon. The lifetime of the triplet state for xenon is significantly shorter than for other noble gases, which makes high pressure xenon a promising gas for a proportional scintillation gas counter [47].

Finally, an important aspect of ionization is the variation in the number of free electrons produced by the incident charged particle. Since the energy of an event will be measured from the number of ionization electrons, this fluctuation will have a direct impact on the energy resolution of the detector. The Fano factor, $F$, characterizes the intrinsic fluctuation in the partitioning of deposited energy between ionization and other mechanisms. The root mean square (rms) fluctuation in the number of free electrons, $N_{i}$, can be expressed as [42] 


$$
\sigma_{i}=\sqrt{F N_{i}}
$$

For gaseous xenon, various measurements have shown that $F_{G X e}=0.15 \pm 0.02$ [42]. In comparison, liquid xenon exhibits a Fano factor of about $F_{L X e} \approx 20$ [42]. This large difference implies that, in principle, by exploiting the ionization signal alone, a gaseous xenon detector can achieve a energy resolution superior by a factor of $\sqrt{F_{H P X e} / F_{L X e}}=0.087$. Since $N_{i}$ and $N_{s}$ are anti-correlated, it is possible to compensate the ionization fluctuation in order to improve the resolution by measuring the number of scintillation photons emitted. This approach was adopted in EXO200. However, the scintillation signal cannot be measured with sufficient precision and accuracy to recover the intrinsic energy resolution [42].

\subsubsection{Electron Drift and Diffusion}

TPCs allow full three dimensional reconstruction of the path taken by a primary charged particle through the detection of the ionization electrons produced along the track. These electrons drift with an average constant velocity due to elastic collisions with the gas molecules. However, these same collisions add an effect of diffusion to the drifting electrons which affects the tracking resolution of the detector. A deep minimum exists in the electron-atom elastic collision cross-section formula called the Ramsauer-Townsend (R-T) minimum. For xenon, this minimum occurs at about $1 \mathrm{eV}[42]$.

The drift properties can be estimated from the classical kinetic theory of gases. In the absence of an electric field or any other external force, an electron in a gas at temperature $T$ possesses a Maxwellian energy distribution with a most probable value $k T$, where $k$ is the Boltzmann constant [49]. This corresponds to about $0.04 \mathrm{eV}$ at room temperature. If an electric field $E$ is added, the electron will have a net 
motion in the direction of the field with a drift velocity given by [49]

$$
w=\frac{e E}{m} \tau
$$

where $\tau$ is the average time between collisions and $e$ and $m$ are the charge and mass of electrons respectively.

When an electron drifts over a distance $x$ with a drift velocity $w$, its diffusion along any direction can be described by a Gaussian distribution with a standard deviation

$$
\sigma=\sqrt{\frac{2 D x}{w}}
$$

where $D$ is the field dependent diffusion coefficient that characterizes the expansion of electron swarm as it drifts along the field lines.

It should be noted that, since the average velocity of the electrons is higher in the direction parallel to the field than the one perpendicular to it, the diffusion is also different in those two directions. Therefore, two diffusion coefficients are used, one longitudinal and the other transverse with respect to the electric field, denoted $D_{L}$ and $D_{T}$ respectively.

In conclusion, the drift and diffusion of electrons in gases can be described by the drift velocity $w$ and the two diffusion coefficients $D_{L}$ and $D_{T}$. These three parameters are electric field and pressure dependent and are different for various gases and gas mixtures. A review of experimental and theoretical data on the transport properties of pure gaseous xenon is presented in Vladimir et al. [50].

\subsubsection{Electroluminescence}

Electroluminescence is a process where photons are produced by electrons drifting in a gas under the influence of an electric field. Below a certain electric field, only 
elastic collisions occur between a drifting electron and the gas atoms. When the field is increased above this threshold, the electron is able to acquire an energy corresponding to the first excitation level of the gas $(8.32 \mathrm{eV}$ for xenon [42]). The electron can then excite the gas atoms though inelastic collisions. As in the production of excitation luminescence described in Equations 2.5 and 2.6, the excited atoms rapidly form excited xenon molecules $X e_{2}^{*}$ through three body collisions, which in turn decay to produce vacuum ultraviolet photons.

Another electric field threshold exists above which the electron can reach a sufficiently high energy to liberate another electron through ionization. This creates an exponential charge multiplication since the newly liberated electrons can also ionize the gas, creating an electron avalanche. This cascade degrades the energy resolution of the detector by producing more EL photons in two ways. Firstly, the new ionization electrons also emit EL photons as they drift. Secondly, the atomic ions created also contribute to the production of EL light through a recombination process like the one described in Equations 2.7 to 2.12 [42]. The range of reduced electric field within which EL can be produced in gaseous xenon without charge multiplication is approximately $1.3 \mathrm{kV} \mathrm{cm}^{-1}$ bar $^{-1}$ to $8 \mathrm{kV} \mathrm{cm}^{-1} \mathrm{bar}^{-1}[51]$.

In XEP, electroluminescence will be exploited for the amplification of the signal. This technique has been widely used for high-resolution spectroscopy of X-ray and low energy gamma-ray $[42,51]$. More recently, it has also been exploited for the search of weakly interacting massive particles (WIMP) through the development of two-phase liquid noble gas detectors $[42,51]$.

The idea consists of dividing the detector in two distinct regions of electric field. In the low-field region, where the fiducial volume is located, free electrons resulting from ionizing events drift toward the high-field region. Once they reach the high-field region, they are converted into an EL light signal which constitutes the basis of the signal amplification. In this region, consisting of a gap of $1 \mathrm{~cm}$ of thickness, the field is 
made as uniform as possible so that all the electrons cross the same electric potential, thus producing a number of EL photons with the smallest fluctuation possible.

The small variation in the gain is what confers to this technique its most important advantage. In fact, EL probably allows a better energy resolution than any other amplification technique in gas [42].

Contradicting data can be found in the literature about the electroluminescence yield in xenon. While data calculated from the Boltzmann equation and obtained using Monte Carlo simulation are in good agreement, the values found in experiments vary significantly from each other [51].

The quantity commonly used is the reduced electroluminescence yield $Y / N$, the electroluminescence yield divided by the number density of the gas. This variable presents an approximately linear relation with the reduced electric field $E / N$. Some recent results, together with the simulation and Boltzmann calculation values, show a good agreement with the equation [51]

$$
Y / N=0.140 E / N-0.474
$$

where $Y / N$ is in $10^{-17}$ photons electron ${ }^{-1} \mathrm{~cm}^{2}$ atom ${ }^{-1}$ and $E / N$ is in $\mathrm{Td}$ $\left(1 \mathrm{Td}=10^{-17} \mathrm{~V} \mathrm{~cm}^{2}\right.$ atom $\left.^{-1}\right)$. This can also be expressed as [51]

$$
Y / P=140 E / P-116
$$

where $Y / P$ is in photons electron ${ }^{-1} \mathrm{~cm}^{-1}$ bar $^{-1}$ and $E / P$ is in $\mathrm{kV} \mathrm{cm} \mathrm{cm}^{-1} \mathrm{bar}^{-1}$. In Favata et al. [52] it was concluded that $\mathrm{Y} / \mathrm{N}$ is independent of the operating pressure. Furthermore, Fonseca et al. [53] have shown that $\mathrm{Y} / \mathrm{N}$ is temperature independent in the range from $20^{\circ}$ down to $-88^{\circ}$. Some experiments found yields of a factor of two 
lower than the one presented above. The different results reported could be due to different levels of gas impurities [51].

\section{$2.3 \quad$ Experimental requirements}

In order to demonstrate whether or not this design of Xe gas TPC will be capable of identifying a $\beta \beta(0 \nu)$ event with a high level of certainty, it will be essential to be able to distinguish such events from all sources the of background. Due to the very long lifetime of ${ }^{136} \mathrm{Xe}$, an extremely high sensitivity is needed, which imposes a very stringent background rejection requirement.

The first defence against background consists obviously in preventing it. For radiation from radioactive isotopes, this is usually performed by shielding the experiment against external sources and by selecting extremely low radioactivity materials for the construction of the detector. However, the effect of such measures are understood and it should be possible to perform the technical study of the XEP prototype without implementing them. Therefore, there is no requirement to make the detector from low radioactivity materials and the external radiation shielding will be limited to shelves of water boxes along the two concrete walls closest to the detector and to steel plates under it.

Two criteria that are to be demonstrated with XEP are the achievement of a high energy resolution and a good tracking ability. The energy resolution is critical for distinguishing double beta decay events from background as well as to separate the $\beta \beta(0 \nu)$ from the $\beta \beta(2 \nu)$ events (see Figure 1.4). A good tracking resolution could allow the recognition of a double beta decay event through the observation of some topological features in the tracks of the emitted electrons. In addition, the ability to detect the daughter barium ion from the double beta decay of ${ }^{136} \mathrm{Xe}$ would allow the confirmation of the $\beta \beta(0 \nu)$ origin of the detected signal and practically suppress 
all background. If a technique to perform this is successfully developed, it could eventually be incorporated to the TPC to demonstrate the entire detection process. For now, however, barium tagging is researched as a separate project.

\subsubsection{Energy resolution}

One of the objectives of XEP is to demonstrate the ability to measure the energy deposited in the TPC with a resolution $\sigma_{E} / E=1 \%$ or better. Since the energy will be measured by counting the number of ionization electrons, a limiting factor for the energy resolution will be the fluctuations in the ionization of the gas, characterized by the Fano factor, as mentioned in Section 2.2.1. Using Equations 2.2 and 2.13, the intrinsic energy resolution can be defined as [42]

$$
\frac{\sigma_{E}}{E}=\sqrt{\frac{F W_{i}}{E}}
$$

Using $W_{I}=21.9 \mathrm{eV}[42]$ and $F=0.15$, the intrinsic resolution at the Q-value of ${ }^{136} \mathrm{Xe}(2480 \mathrm{keV})$ is $\sigma_{E} / E=1.2 \times 10^{-3}$, which is better then the required resolution.

With the electroluminescence process employed to detect the electrons, it is reasonable to assume that the detection of individual electrons are uncorrelated. Therefore, if $N_{i}$ is the number of ionization electrons formed in the TPC and $G$ represents the variance of the signal gain from the detection of an electron, the standard deviation for the detection process due to signal variation is [42]

$$
\sigma_{N_{i}}=\sqrt{G N_{i}}
$$

It can also be assumed that the fluctuations in the detection of electrons are uncorrelated from those in the ionization of the gas, so that the total fluctuation of the signal can be written as [42] 


$$
=\sigma_{N_{i}}=\sqrt{(F+G) N_{i}}
$$

Then, the energy resolution can be expressed as [42]

$$
\frac{\sigma_{E}}{E}=\sqrt{\frac{(F+G) W_{i}}{E}}
$$

To achieve the required resolution, $G$ should be at most on the order of $F$. Otherwise, the advantage of the small Fano factor in gaseous xenon would be lost. Again, electroluminescence provides a low fluctuation process for the amplification of the ionization signal and should allow to meet this requirement.

Some of the main contributions to the variance of the detected signal should be the number of detected EL photons per ionization electron and the gain process in the $\mathrm{CH}_{4}$ gas. Let $n_{E L}$ be the number of EL photons emitted per electrons, $n_{p e}$ be the number of photoelectrons produced on the CsI surface per incident electron, and $\sigma_{p d}^{2}$ be the variance of the gain process of the photodetector. To a good approximation, the variance $G$ can be given as $[42,54]$

$$
G=\frac{J}{n_{E L}}+\frac{1+\sigma_{p d}^{2}}{n_{p e}}
$$

where $\mathrm{J}$ is a Fano-like factor defined by $\sigma_{n_{E L}}^{2}=J n_{E L}$. The first term of Equation 2.22 represents the variations in the number EL photons produced per ionization electrons, the $1 / n_{p e}$ term characterizes the variation of the number of these photons that reach the photocathode, and the $\sigma_{p d}^{2} / n_{p e}$ term characterizes the fluctuations in the gain process in the $\mathrm{CH}_{4}$.

The parameter $J$ characterizes the fluctuations in the energy loss by the electrons in excitation and in elastic collisions. The fluctuations in $n_{E L}$ should also be affected 
by the non-uniformity of the electric field in the EL region, as well as by charge multiplication that might occur near the electrodes of the electric field, where the field intensity becomes higher and the energy of the drifting electrons might reach the ionization potential of the gas. The value of $J$, which decreases with increasing $E / p$, has been found to be as low as 0.02 at $E / p=7 \mathrm{kV} \mathrm{cm}^{-1} \mathrm{bar}^{-1}$ [54].

However, the second term in Equation 2.22 will typically be much higher than the first one due to the low photon detection efficiency. Also, several other effects will likely affect the energy resolution by introducing fluctuations in other part of the detection process. For example, ionization electrons can be lost due to electron capture caused by electronegative impurities in the gas. Additional fluctuations will likely arise from the UV light attenuation in xenon gas and from electronic noise.

\subsubsection{Tracking}

An accurate reconstruction of the track can provide a powerful tool to recognize double beta decays and to reject background events. Because of multiple scattering, the tracks are likely to be very convoluted and reconstructing them accurately might be difficult. The ability to do this will certainly be limited by the diffusion of the ionization electrons drifting in the gas as well as the dimension of the readout pads. However, it will be useful to determine as much as possible about the tracks in order to identify and reject background events.

One of the features that would be useful to observe is the Bragg peak, a pronounced peak in the plot of the energy loss by a charged particle as a function of the distance travelled through matter. The peak occurs because the ionization interaction cross section of the incident particle increases as its energy decreases. Hence, a higher ionization concentration should normally appear at the end of a track. Identifying the Bragg peaks would allow the differentiation of events formed by two charged particles, such as double beta decay, from single particle events, which are necessarily 
background.

Even with the observation of Bragg peaks, two electrons of background origins could be mistaken for a double beta decay event if they pass sufficiently close to each other. A good tracking resolution would facilitate the separation of tracks with a common origin from multi-site events that are necessarily background events. Furthermore, observing the location of the energy deposition along the track could help in identifying the type of particle involved due to the different stopping power $(\mathrm{dE} / \mathrm{dx})$ of the various particles. For particles observed near the edge of the detector, a good tracking resolution could clarify whether or not their tracks are fully located within the fiducial volume. This is essential to determine since if the track is not fully contained, it is impossible to measure its energy precisely.

Finally, being able to locate the events vertex would have multiple advantages. First, if a particle originates at the surface of the detector materials, then it is necessarily background. Second, the density of background tracks originating near the edge of the TPC should be higher than near the center, while the double beta decay events should be distributed uniformly throughout the detector. Third, knowledge of the vertex of a double beta decay event would be useful for the design of the barium tagging system since it would indicate the location of the $\mathrm{Ba}^{++}$ion. 


\section{Chapter 3}

\section{XEP Detector Simulation}

A Monte Carlo simulation of the XEP detector was developed to simulate ionizing events in the TPC, the production and propagation of the light signal from those events and its detection on the CsI readout plane. The simulation was developed using the Geant4 toolkit [36] and the Magboltz [37] and Garfield [38] programs.

A Geant4 application constitutes the main part of this simulation. Geant4, which stands for GEometry ANd Tracking, is a software toolkit for the simulation of the passage of particles through matter. It provides detailed detector modelling capabilities and a comprehensive set of physics models to describe the interactions of particles with matter, including the production of secondary particles.

Although Geant4 can calculate the energy deposition of particles in xenon, it is unable to produce certain processes in the gas, such as the production of ionization electrons and their resulting drift inside the chamber under the influence of the electric field. On the other hand, Garfield is a FORTRAN program designed specifically for the simulation of drift chambers and gas detectors. Therefore, it was a natural choice for simulating the ionization electron's drift toward the electroluminescence region. To accomplish this task, the program requires the drift velocity and diffusion coefficients of electrons in pure xenon. For this, Garfield provides a seamless interface with Magboltz, a program designed to calculate the transport properties of electrons 
in gas mixtures under the influence of an electric field by solving the Boltzmann transport equations.

For this simulation, an interface between Geant 4 and Garfield was created. The primary use of Geant 4 is the simulation of the interactions of particles with the various components of the detector, including the xenon gas, as well as the propagation of the light signal within the detector. From the energy deposited by charged particles in the gas computed by Geant4, the number and distribution of ionization electrons is calculated. This information is passed to Garfield, which calculates the diffusion and arrival time of each electrons at the electroluminescence region. Geant 4 is then used to generate and propagate the resulting electroluminescence light and calculate its detection on the two photocathode planes.

\subsection{The Geant4 Application}

\subsubsection{Physics Processes}

Constructing a Geant4 application requires specifying the physical processes that describe the interactions of the particles with the materials. In this simulation, the following electromagnetic processes were included: multiple scattering, ionization, bremsstrahlung, annihilation, pair production, Rayleigh scattering, photoelectric effect, and Compton scattering. Whenever available, the low-energy electromagnetic models were used, which can describe the interaction of electrons and photons with matter down to $250 \mathrm{eV}$ using interpolated data tables based on the Livermore library [55]. In addition, processes for optical photons were also included, including scintillation, Cerenkov radiation, absorption, Rayleigh scattering, and boundary processes.

Furthermore, the Radioactive Decay Module (RDM) [56] was implemented for the 
simulation of the nuclear radiation. The RDM is an extension to GEANT4 developed under the sponsorship of the European Space Agency. It allows simulation of the radioactive decay of isotopes over multiple nuclide generations and to generate the products of the decays (i.e., alpha and beta particles and gamma rays) with the correct energies and intensities. It uses the Evaluated Nuclear Structure Data File (ENSDF) [57] for determining the branching ratios of radioactive decays.

Finally, two functions were added to the code for the generation of the two electrons emitted in neutrinoless and two-neutrino double beta decay events with the appropriate energy and angular distributions. These functions were adapted from the EXO-200 Geant4 simulation. The ground state to ground state transition $\left(0^{+} \rightarrow 0^{+}\right)$ is assumed as described in F. Boehm \& P. Vogel, 1992, pp.145-151. [58]. The energies of both electrons, $E_{1}$ and $E_{2}$, are randomly generated to satisfy the phase space constraint proportional to [58]

$$
d G^{0 \nu} \sim p_{1} E_{1} F\left(E_{1}\right) p_{2} E_{2} F\left(E_{2}\right) d E_{1} d E_{2}
$$

where $p_{1}$ and $p_{2}$ are the momentum of the two electrons and $F(E)$ is the Fermi function that describes the Coulomb effect between the emitted electrons and the final nucleus. The angle $\theta$ between the electrons is also randomly selected according to a distribution of the form [58]

$$
f(\cos \theta)=1-\frac{p_{1}}{E_{1}} \frac{p_{2}}{E_{2}} \cos \theta .
$$

\subsubsection{Geometry}

A number of classes provided with Geant4 allow the geometry of the simulated detector to be accurately described. The geometry of the XEP TPC that was modelled 

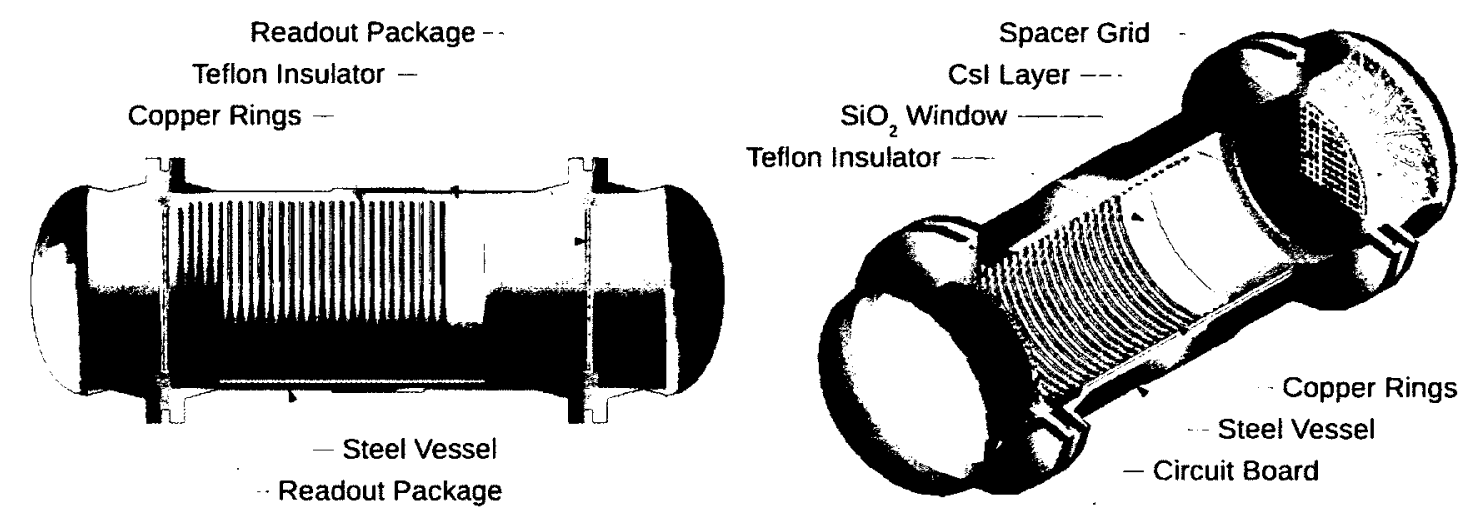

Figure 3.1: Visualization of the detector modelled in Geant4.

is shown in Figure 3.1. The model includes the stainless steel vacuum vessel, the gaseous xenon, the Teflon insert and the 27 copper rings for the drift electric field. It also includes the two readout packages, each comprising a fused silica window, a methane gap with the Teflon spacer grid, a thin layer of CsI and a fibreglass circuit board. Furthermore, a second volume of xenon is included inside the one previously mentioned to serve as the active xenon volume. It is only for the ionizing events occurring in this volume that the deposition of ionization electrons is calculated. It extends from the position of the anode mesh to the last copper ring and has a diameter of $49.53 \mathrm{~cm}$, which corresponds to a distance of $5.08 \mathrm{~cm}$ from the chamber inner wall and $1.34 \mathrm{~cm}$ from the copper rings. Finally, a uniform electric field is included in the active xenon volume.

In addition to the detector, some elements of the cleanroom in which the detector will operate were included for the simulation of background radiation. These components, illustrated in Figure 3.2, are the concrete floor, ceiling and the two closest walls to the detector, the rows of water boxes along the walls and the steel plates on the floor used for shielding, as well as the air that fills the room.

All the dimensions of the detector and the room were taken from the technical 


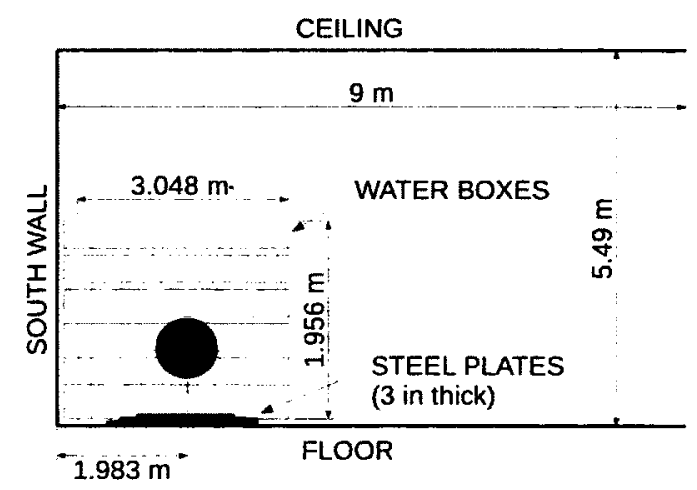

(a) View facing west

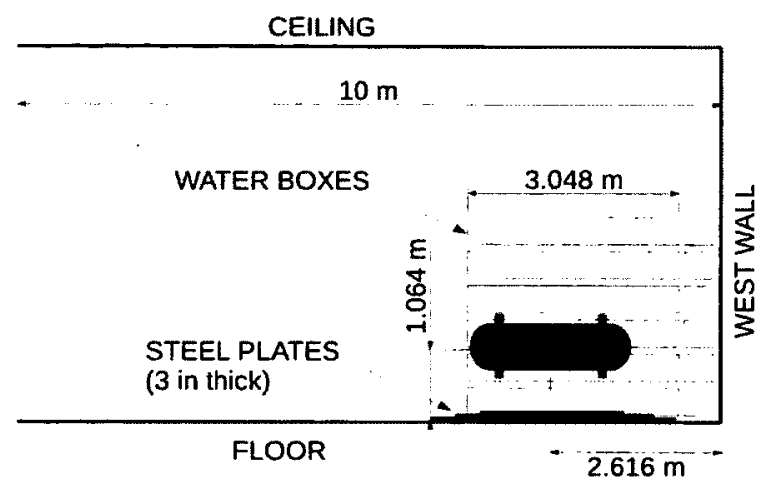

(b) View facing south

Figure 3.2: Illustration of the components of the room where the detector is being built that were included in the simulation.

drawings provided by Matt Bowcock and Vance Stickland, who are respectively designer and engineer collaborating with the EXO experiment at Carleton University. The xenon gas was given a temperature of $300 \mathrm{~K}$ and the pressure was generally chosen between 2,5 , or $10 \mathrm{~atm}$. The density corresponding to each of these pressures was calculated by solving the Van der Waals equation

$$
\left(P+\frac{n^{2} a}{V^{2}}\right)\left(\frac{V}{n}-b\right)=R T
$$

where $\mathrm{P}$ is the pressure, $\mathrm{V}$ is the volume, $\mathrm{n}$ is the number of moles, $a=4.137 \times 10^{-6}$ atm $\mathrm{m}^{6} \mathrm{~mol}^{-2}$ and $b=5.16 \times 10^{-5} \mathrm{~m}^{3} \mathrm{~mol}^{-1}$ are the two Van der Waals constants, and $R=8.206 \times 10^{-5} \mathrm{~m}^{3}$ atm $\mathrm{K}^{-1} \mathrm{~mol}^{-1}$ is the universal gas constant [59]. For the three pressures mentioned above, this formula gives values of $0.0108 \mathrm{~g} \cdot \mathrm{cm}^{-3}, 0.0273 \mathrm{~g} \cdot \mathrm{cm}^{-3}$, and $0.0561 \mathrm{~g} \cdot \mathrm{cm}^{-3}$, respectively. At standard temperature and pressure (STP), it gives a density of $0.00590 \mathrm{~g} \cdot \mathrm{cm}^{-3}$, close to the value of $0.005887(9) \mathrm{g} \cdot \mathrm{cm}^{-3}$ quoted in the CRC Handbook of Chemistry and Physics, 87th edition [59]. The final operating pressure of the methane gas between the window and the CsI layer was not known at the time when this work was done, so a temperature of $300 \mathrm{~K}$ and a pressure of 0.1 atmosphere were assumed, which correspond to a density of $6.627 \times 10^{-5} \mathrm{~g} \cdot \mathrm{cm}^{-3}$ 
using the ideal gas law:

$$
P V=n R T,
$$

where the variables are the same as in Equation 3.3.

\subsubsection{Scintillation}

The primary scintillation light generated by charged particles passing through the xenon gas will be important for XEP to mark the starting time of the charge collection. In Geant4, the scintillation process is characterized by a number of empirical parameters. The "scintillation yield" represents the mean number of photons emitted per energy deposited in the scintillating material. The actual number of photons produced in a given step fluctuates around this mean number, $\bar{N}_{\gamma}$, with a width $\sigma=R \sqrt{N_{\gamma}}$, where $R$ is the intrinsic "resolution scale" for the scintillating material. The material also has a time spectrum given by two exponential decay time constants, the "fast" and "slow components", which describe the decay times of the $\mathrm{Xe}_{2}^{*}$ excimer in the singlet state, ${ }^{1} \Sigma_{u}^{+}$, and triplet state, ${ }^{3} \Sigma_{u}^{+}$, respectively. The photons are generated uniformly along the step length with isotropic direction and random linear polarization. The scintillation parameters used in this simulation are listed in Table 3.1. For now, the scintillation was assumed to follow normal fluctuations (i.e., $\sigma=\sqrt{N_{\gamma}}$.

The photons produced by the scintillation process are then propagated through the detector. They can be detected when they reach the surface of the CsI layer which has a $20 \%$ detection efficiency.

Before reaching the CsI layer, the photons have to travel through the xenon gas, the fused silica window, and the methane gap. When passing through the window, they can undergo absorption, which is characterized by the "absorption length", the 


\begin{tabular}{ccc} 
Parameter & Value & source \\
\hline Scintillation Yield & $13889 \mathrm{MeV}^{-1}$ & {$[45]$} \\
Resolution Scale & 1 & \\
Fast Component & $4.6 \mathrm{~ns}$ & {$[48]$} \\
Slow Component & $99 \mathrm{~ns}$ & {$[48]$}
\end{tabular}

Table 3.1: Material optical properties used in Geant4.

average distance travelled by photons before being absorbed. The window's absorption length was set to $9.161 \mathrm{~mm}$, which means that a photon traversing the window perpendicularly to its surface has $50 \%$ probability of being absorbed. This value was motivated by the work of Christian Ouellet, one of the collaborators of the EXO research group at Carleton, who studied the properties of a sample of the same material that will be used to produce the window.

When a photon is incident on the surface of an opaque material, it can be either absorbed or reflected, which is characterized by a reflectivity ratio. When a photon is incident to the boundary between two transparent volumes (such as either side of the fused silica window), the photon can be subject to Fresnel reflection, refraction, or total internal reflection. These processes depend only on the index of refraction of the medium on both side interface, the photon's wavelength, and the incidence angle.

In every case, the direction of the reflected or refracted photon is influenced by the finish of the surface. A "polished" finish represents a perfectly smooth surface while a "ground" finish represents a rough surface. In the latter case, a value of "polish" can be specified. The surface can be imagined as made of multiple micro-facets with normals pointing in different directions. When a photon is incident to the surface, the micro-facet's normal is calculated by adding to the average surface's normal a vector of magnitude (1-polish) with random direction. A polish of zero means maximum 
roughness with the angle, $a$, between the micro-facet normal and that of the average surface distributed as $\cos (a)$.

UV light around $175 \mathrm{~nm}$ will likely be absorbed by all the surfaces in the detector except the Teflon surface, which was given a reflectivity of 0.9 [60] and a ground finish with a polish value of zero. The fused silica window, which has a frosted surface, was also given a ground finish with a polish value of zero. Finally, the index of refraction of the xenon, the fused silica and the methane were set to 1.0011, 1.561, and 1.00044, respectively [61]. More information about the optical processes in Geant4 can be found in Reference [55] and [62].

It should be noted that several effects are not considered in this simulation. For example, the two wire meshes that produce the EL region electric field as well as the wire mesh in the $\mathrm{CH}_{4}$ gap would provide additional shadowing of the light signal which would reduce the number of photons detected. Furthermore, the efficiency of the CsI pads is assumed to be perfectly uniform.

\subsection{Ionization}

The electron ionization process in Geant 4 provides the ionizing energy deposition from charged particles travelling through the xenon gas. Above a certain threshold energy (990 eV by default), the energy loss is simulated by the explicit production of delta rays by Möller scattering [62]. Below this threshold, the soft electron ejected are simulated as continuous energy loss by the incident particle. Therefore, Geant4 does not explicitly produce every ionization electron that would be emitted in reality.

To calculate the number of ionization electrons produced, the ionizing energy $E_{i}$ deposited in a given step $i$ is divided by the average energy loss required to produce a charge pair, $W=22 \mathrm{eV}$, to obtain the mean number of charges in that step $\bar{N}_{i}$. Then, to account for the intrinsic fluctuation of the number of electron produced, a number 
is randomly selected from a Gaussian distribution with a mean $\bar{N}_{i}$ and a standard deviation $\sqrt{F \bar{N}_{i}}$, where $F$ is the Fano factor, as in Equation 2.13. This number is then rounded to the closest integer to give the number of ionization electrons deposited in this step. The excess energy, if the number was rounded down, or the missing energy, if it was rounded up, is given to or taken from the next step of the track, ensuring that over the whole track, the rounding error on the total number of charges produced is no more than one electron. The electrons created via ionization are then deposited uniformly along the step, and the position and time of each electron are recorded in a file to be used by Garfield.

\subsection{Electron Transport with Garfield and Mag- boltz}

After each event in Geant4, Garfield is launched to calculated the arrival time and position of each electron in the EL region. The electric field is assumed to be uniform and extends from the position of the anode mesh to the last copper ring.

Before starting the drift simulation, Garfield must obtain from Magboltz the electron drift velocity and diffusion coefficients. All Magboltz requires is the gas mixture (pure xenon in this case) and the gas pressure and temperature. The transport properties are then calculated for a sample of electric field strengths, which were chosen as 20 values between 0.1 and $2 \mathrm{kV} / \mathrm{cm}$. Between those values, interpolation is used to determine the transport properties. The results are saved in a "gas table" that Garfield can access. Since Magboltz is computationally very intensive, if the gas table was already computed for the required temperature and pressure, Garfield simply accesses the gas table from the file where it is saved. Otherwise, it asks Magboltz to generate the table. 


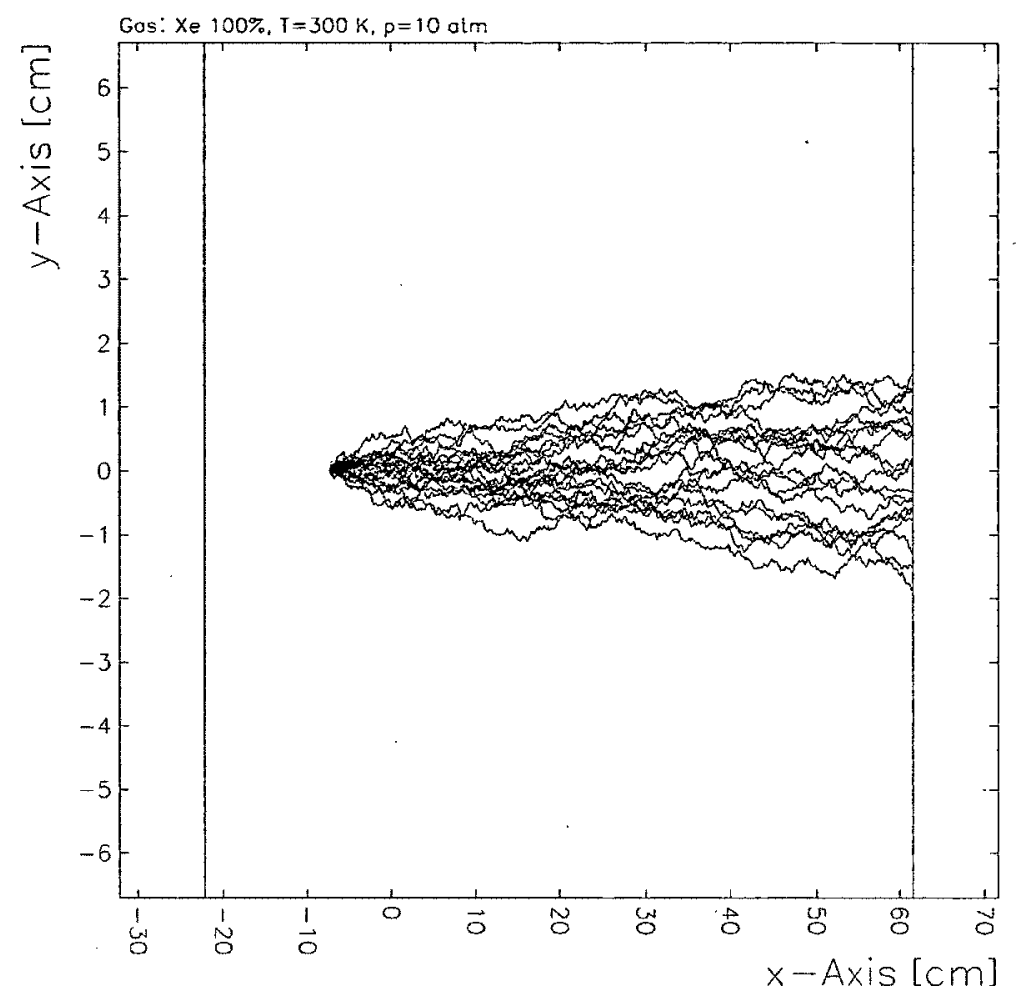

Figure 3.3: Plot from Garfield showing 20 electrons drifting from the same point over $60 \mathrm{~cm}$ in 10 atmosphere xenon at $300 \mathrm{~K}$ under the effect of a $1 \mathrm{kV} / \mathrm{cm}$ uniform electric field.

With the Magboltz results, Garfield can simulate the electron drift toward the electroluminescence region. For this, it use a Monte Carlo method which iterates over a series of steps to calculate the transverse and longitudinal diffusion. The step size was set to $1 \mathrm{~mm}$. At the end, Garfield provides the position and time at which each ionization electron reaches the position of the anode grid. Figure 3.3 shows an example of the drift and diffusion of electrons produced in Garfield.

To verify the validity of the results from Garfield, a test was made where the drift velocity $w$ and the longitudinal and transverse diffusion coefficients $D_{L}$ and $D_{T}$ were calculated from Garfield's output and compared with reported values. For this, 100,000 electrons were drifted in xenon at various pressures from the same point over a distance $L=70 \mathrm{~cm}$. The transport properties were calculated from the arrival time 
and position of each electron at the anode position. Assuming that the arrival time spectrum can be approximated as a Gaussian distribution, it can be shown that [63]

$$
w \approx L / t_{m}
$$

where $t_{m}$ is the mean arrival time. Furthermore, it can also be shown that [63]

$$
D_{L} \approx L^{2} \sigma_{t}^{2} / 2 t_{m}^{3}
$$

where $\sigma_{t}^{2}$ is the variance of the arrival time spectrum. Finally, $D_{T}$ was approximated as

$$
D_{T} \approx\left(\sigma_{x}^{2}+\sigma_{y}^{2}\right) w / 4 L
$$

where $\sigma_{x}^{2}$ and $\sigma_{y}^{2}$ are the variance of the $x$ and $y$ distribution of the electron on the anode plane. The different distributions of the arrival time and position on the anode plane were fitted with a Gaussian function and the fit parameters $\bar{t}, \sigma_{t}^{2}, \sigma_{x}^{2}$, and $\sigma_{y}^{2}$ were used to calculate the values of $w, D_{L}$ and $D_{T}$ listed in Table 3.2.

As can be seen, the calculated values are in reasonable agreement with the reported ones. The longitudinal diffusion coefficient seems however higher then the ones reported in the literature [50], but the difference does not cause a significant effect on the results. In the future, the diffusion coefficients calculated with Magboltz which are used in this simulation could be changed to the reported ones. 


\begin{tabular}{ccc} 
& Calculated Values & Reported Values \\
\hline$w$ & $114503 \pm 4 \mathrm{~cm} \mathrm{~s}^{-1}$ & $110000 \mathrm{~cm} \mathrm{~s}^{-1}[64]$ \\
$D_{L}$ & $388.9 \pm 1.7 \mathrm{~cm}^{2} \mathrm{~s}^{-1}$ & $140 \mathrm{~cm}^{2} \mathrm{~s}^{-1}[50]$ \\
$D_{T}$ & $2034 \pm 4 \mathrm{~cm}^{2} \mathrm{~s}^{-1}$ & $2300 \mathrm{~cm}^{2} \mathrm{~s}^{-1}[50]$
\end{tabular}

(a) at $2 \mathrm{~atm}$ and $0.2 \mathrm{kV} / \mathrm{cm}$

\begin{tabular}{ccc} 
& Calculated Values & Reported Values \\
\hline$w$ & $116405 \pm 2 \mathrm{~cm} \mathrm{~s}^{-1}$ & $110000 \mathrm{~cm} \mathrm{~s}^{-1}[64]$ \\
$D_{L}$ & $149.0 \pm 0.7 \mathrm{~cm}^{2} \mathrm{~s}^{-1}$. & $57 \mathrm{~cm}^{2} \mathrm{~s}^{-1}[50]$ \\
$D_{T}$ & $741 \pm 12 \mathrm{~cm}^{2} \mathrm{~s}^{-1}$ & $930 \mathrm{~cm}^{2} \mathrm{~s}^{-1}[50]$ \\
& $\left(\right.$ b) at 5 atm and $0.5 \mathrm{kV} / \mathrm{cm}^{2}$ \\
& Calculated Values & Reported Values \\
\hline$w$ & $104601 \pm 2 \mathrm{~cm} \mathrm{~s}^{-1}$ & $110000 \mathrm{~cm} \mathrm{~s}^{-1}[64]$ \\
$D_{L}$ & $70.4 \pm 0.3 \mathrm{~cm}^{2} \mathrm{~s}^{-1}$ & $28 \mathrm{~cm}^{2} \mathrm{~s}^{-1}[50]$ \\
$D_{T}$ & $423 \pm 17 \mathrm{~cm}^{2} \mathrm{~s}^{-1}$ & $420 \mathrm{~cm}^{2} \mathrm{~s}^{-1}[50]$
\end{tabular}

(c) at $10 \mathrm{~atm}$ and $1 \mathrm{kV} / \mathrm{cm}$

Table 3.2: Transport properties of electrons in gaseous $\mathrm{Xe}$ at $300 \mathrm{~K}$ calculated from the results of the Garfield simulation of 100,000 electrons drifted over $70 \mathrm{~cm}$ under various pressures and with a uniform reduced electric field of $0.1 \mathrm{kV} \mathrm{cm}^{-1}$ atm $^{-1}$. For comparison, experimental values of the transport properties reported from other works are presented. 


\subsection{Electroluminescence}

The emission of electroluminescence photons is simulated in Geant 4 for each ionization electron drifted with Garfield. The number of emitted photons per centimetre is given by Equation 2.17:

$$
\frac{d N_{E L}}{d x}=(140 E / p-116) p
$$

where $p$ is the gas pressure in bar and $E / p$ is the reduced electric field in $\mathrm{kV} \mathrm{cm}{ }^{-1} \mathrm{bar}^{-1}$. For a reduced electric field of $7 \mathrm{kV} \mathrm{cm}^{-1} \mathrm{~atm}^{-1}$ under a pressure of $10 \mathrm{~atm}$, it gives a number of 8625 photons generated along the $1 \mathrm{~cm}$ gap. For the same reduced electric field with $14 \mathrm{kV} \mathrm{cm}^{-1}$ at $2 \mathrm{~atm}$, the value goes down to 1725 photons per electron. At a lower reduced electric field of $4 \mathrm{kV} \mathrm{cm}^{-1} \mathrm{~atm}^{-1}$, the number of photons per electron would be 4425 at $10 \mathrm{~atm}$ and 885 at $2 \mathrm{~atm}$. Since it is very computationally intensive to track so many photons, this number is usually scaled down in the results presented in this thesis.

The electric field in the electroluminescence region is also assumed to be uniform, and the electrons are considered to be drifting in straight lines towards the fused silica window. According to the literature, the drift velocity of electrons in gaseous xenon seems constant for given values of reduced electric fields [65]. In Brooks et. al. (1982) [66], the drift velocity is reported as $1.48 \times 10^{6} \mathrm{~cm} / \mathrm{s}$ at $E / N=27.9 \mathrm{Td}$, which corresponds to an electric field of $35 \mathrm{kV} \mathrm{cm}^{-1}$ at a pressure of $5 \mathrm{~atm}$. The value of the drift velocity in the EL region should not have a significant impact on the results presented in this thesis. Therefore, the above value of drift velocity was used for the different reduced electric fields considered. The position and time returned by Garfield serve as the starting point of this line and the photons are generated in Geant4 uniformly along it in a random direction. The tracking of these photons within the detector is identical to the tracking of the primary scintillation as described 
in Section 3.1.3.

\subsection{Examples of Generated Tracks}

To illustrate the possible outputs of the simulation, this section presents examples of simulated tracks. To begin, Figures 3.4 and 3.5 show an example of a $1 \mathrm{MeV}$ electron simulated in xenon at 5 atm and $300 \mathrm{~K}$ under a $1 \mathrm{kV} / \mathrm{cm}$ electric field. The track starts at $\mathrm{x}=\mathrm{y}=0$ and $\mathrm{z}=61.6 \mathrm{~cm}$ from the anode mesh position. To make the simulation less computationally intensive, the number of ionization electron was reduced by a factor of 10 . Furthermore, the number of EL photons per electron was reduced to only 43. If an EL region reduced electric field of $4 \mathrm{kV} \mathrm{cm}^{-1} \mathrm{~atm}^{-1}$ is assumed, this would correspond to reducing the EL photon yield by a factor of 51.4.

In Figure 3.4, the three plots of the top row show the EL photon distribution on photocathode $\mathrm{A}$ (the one on the anode side). The values of the $\mathrm{z}$-axis were obtained by multiplying the arrival time of the photons with the expected drift velocity taken from Table 3.2. In the three plots of the lower row, the black line shows the initial electron track. The red dots represent the position of the ionization electrons after they drifted down to the anode mesh position, thus showing the effect of the diffusion during the electrons transport. For these ionization electrons, the z-axis values were also obtained by multiplying the arrival time by the drift velocity. Figure 3.5 shows the arrival time spectrum of EL photons on photocathodes A (on the EL region side) and $\mathrm{B}$ (on the cathode side).

Figures 3.6 and 3.7 present an example of a neutrinoless double beta decay event simulated in xenon at 5 atm and $300 \mathrm{~K}$ under the influence of a $1 \mathrm{kV} / \mathrm{cm}$ drift field. The vertex is located at $\mathrm{x}=\mathrm{y}=0$ and $\mathrm{z}=40 \mathrm{~cm}$ from the anode mesh position. In this specific case, the two emitted electrons had respectively $1057.64 \mathrm{keV}$ and $1400.16 \mathrm{keV}$. The ionization and optical gain were reduced in the same way as for the $1 \mathrm{MeV}$ electron example. 

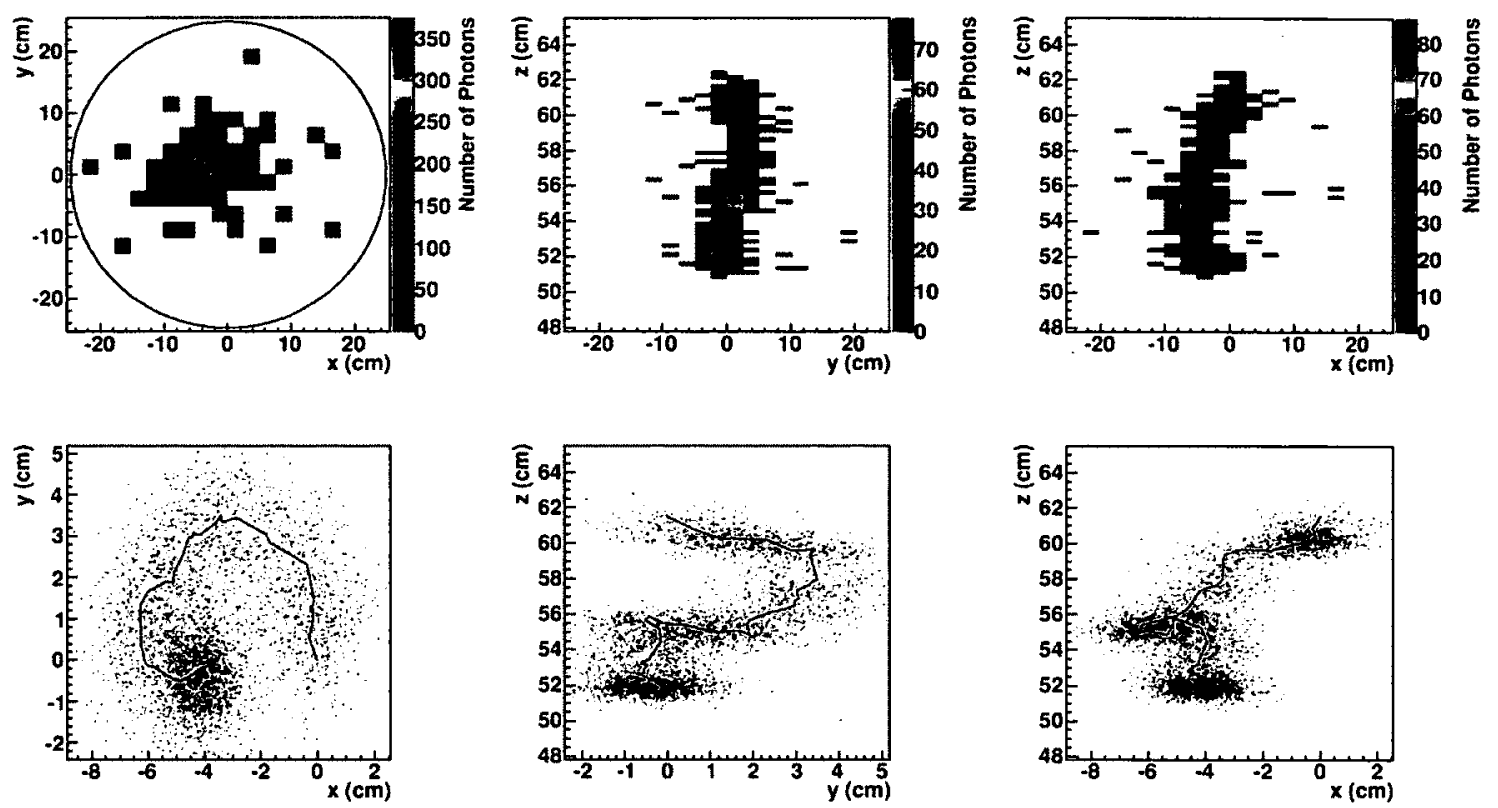

Figure 3.4: Example of a $1 \mathrm{MeV}$ electron track in xenon at 5 atm and $300 \mathrm{~K}$ with a field of $1 \mathrm{kV} / \mathrm{cm}$. The top three plots show the EL photon distribution on the photocathode on the anode side. The three lower plots present the initial track and the diffused ionization electrons after their drift to the anode mesh position.
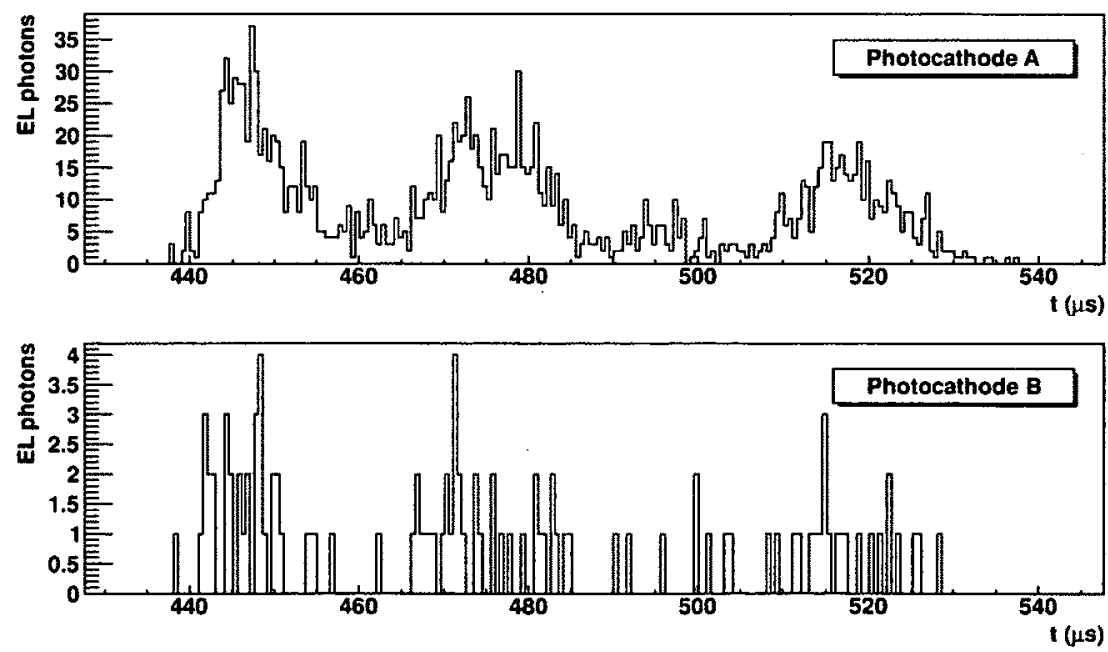

Figure 3.5: Time spectrum of the photons arrival time on the two photocathodes for the track presented in Figure 3.4. It should be noted that to simulate this track, the number of ionization electrons and EL photons had to be scaled down. If a EL electric field of $4 \mathrm{kV} \mathrm{cm}^{-1} \mathrm{~atm}^{-1}$ is assumed, 514 times more photons should be observed. 

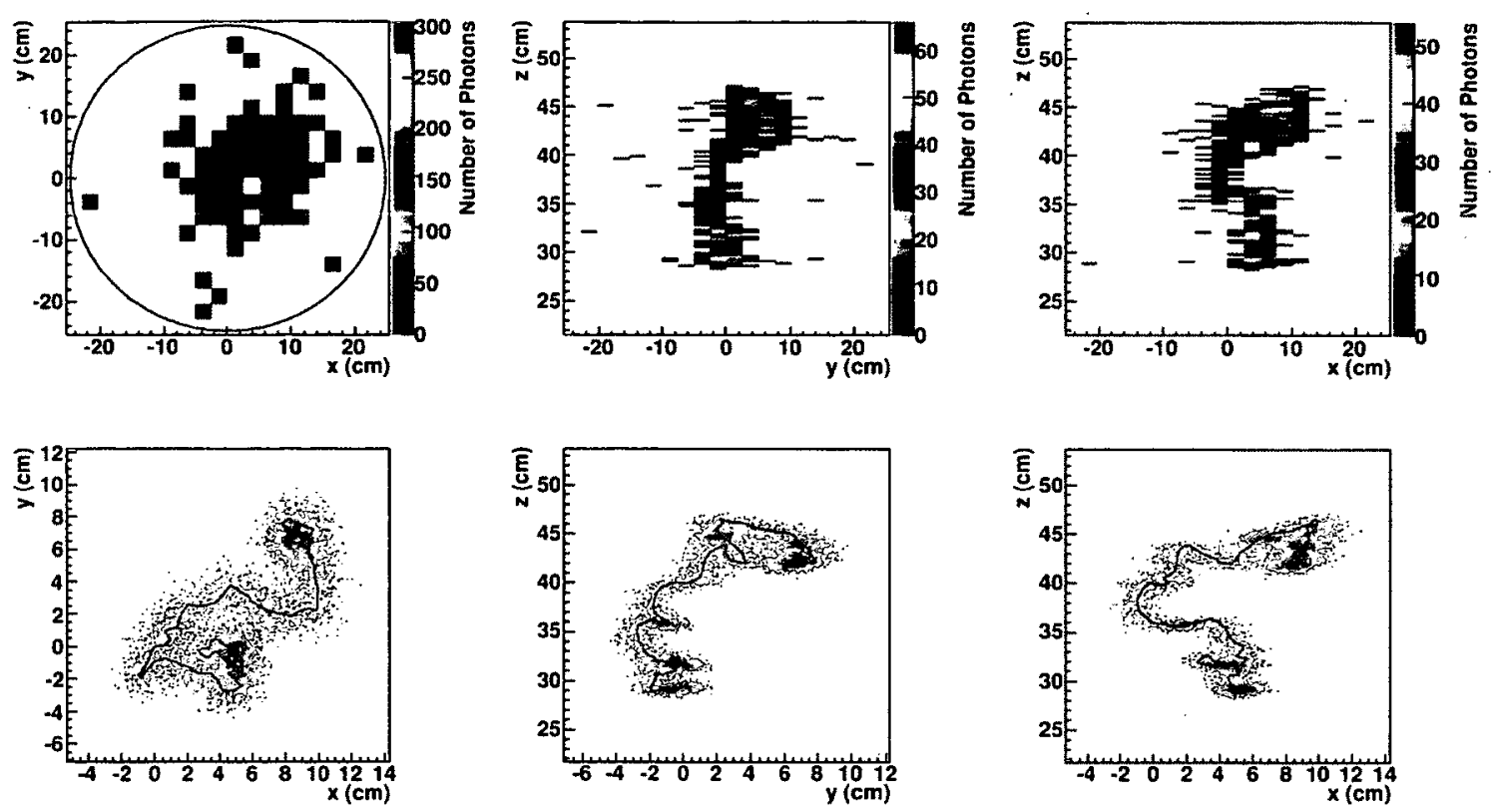

Figure 3.6: Example of a $\beta \beta(0 \nu)$ event simulated in xenon at 5 atm and $300 \mathrm{~K}$ with a field of $1 \mathrm{kV} / \mathrm{cm}$. The top three plots show the photon distribution on the photocathode on the anode side. The three lower plots present the initial track and the diffused ionization electrons after their drift to the anode mesh position.
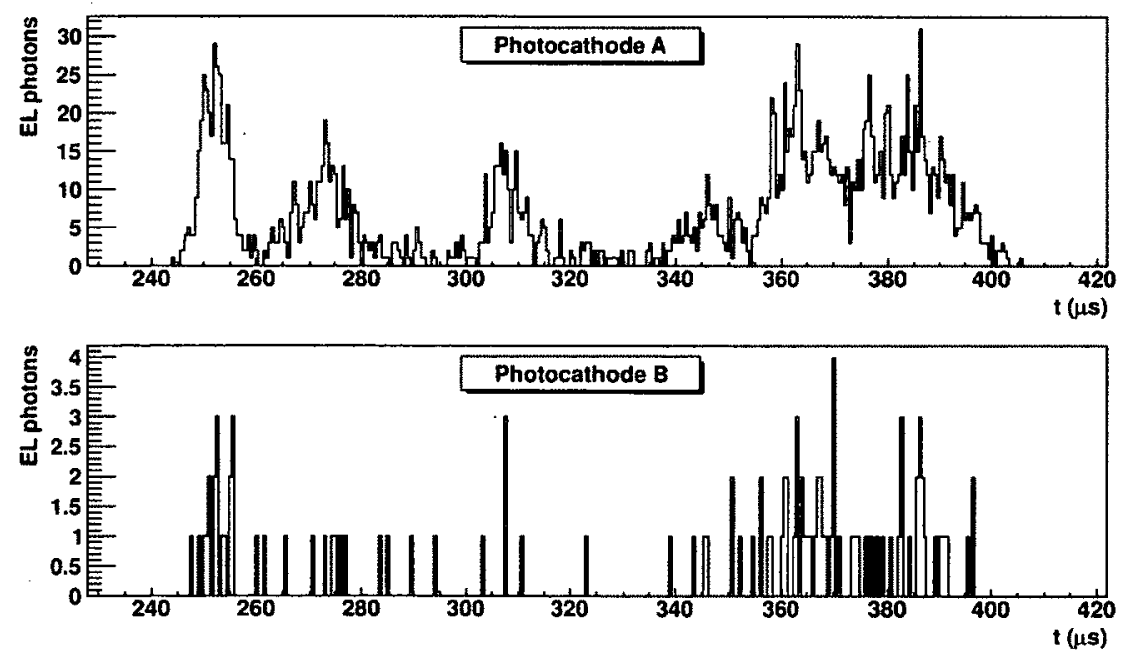

Figure 3.7: Time spectrum of the photons arrival time on the two photocathodes for the track presented in Figure 3.6. As in the previous example, the ionization and EL yield were scaled down. Assuming an EL region electric field of $4 \mathrm{kV} \mathrm{cm}^{-1} \mathrm{~atm}^{-1}$, 514 times more photons should be observed. 


\section{Chapter 4}

\section{Simulation Results}

\subsection{Detection Process Fluctuations}

To test the various steps of the signal generation process, 20,000 electron tracks were simulated to observe the distribution of energy deposited in the gas, the number of scintillation photons, ionization electrons, and EL photons generated, and the number of EL photons detected on each photocathode. The electrons were simulated in gaseous xenon at a pressure of $10 \mathrm{~atm}$ and a temperature of $300 \mathrm{~K}$. An electric field of $1 \mathrm{kV} / \mathrm{cm}$ was present in the drift region while $40 \mathrm{kV} / \mathrm{cm}$ was used for the EL electric field. Only one tenth of the ionization was produced and the number of EL photons per electrons was reduced by a factor of 50. An energy cut was applied to reject the tracks that were not entirely contained inside the xenon active area and 18,526 events were kept. The distributions, which are plotted in Figure 4.1, were fitted with a Gaussian function and the fit parameters are presented in Table 4.1.

The fluctuation in the total energy deposited in the gas by each primary electrons, shown in Figure 4.1a, is due to the electric field simulated by Geant4, which slightly accelerates the electrons toward the anode. When the displacement of the particle in a step of the simulation has a component in the direction of the electric field, the electron gains some kinetic energy, while when it moves in the opposite direction, it 


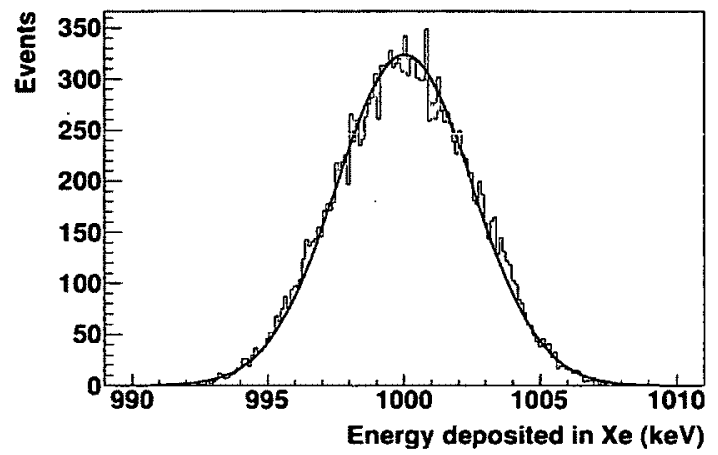

(a)

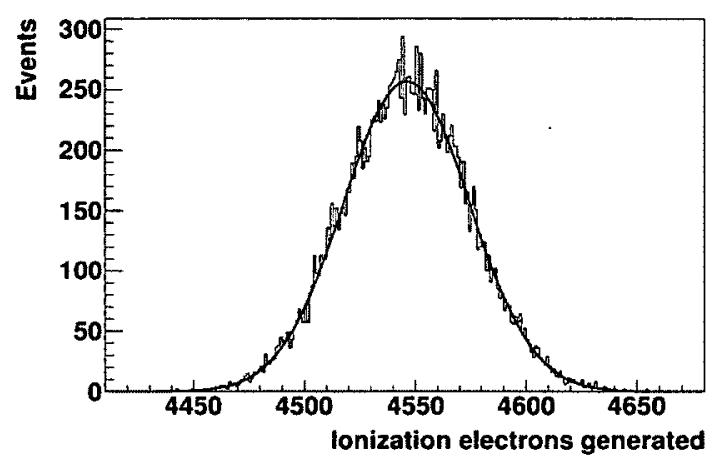

(c)

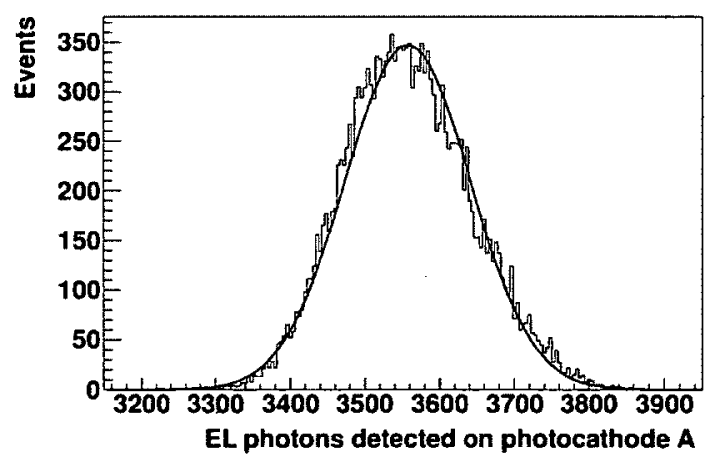

(e)

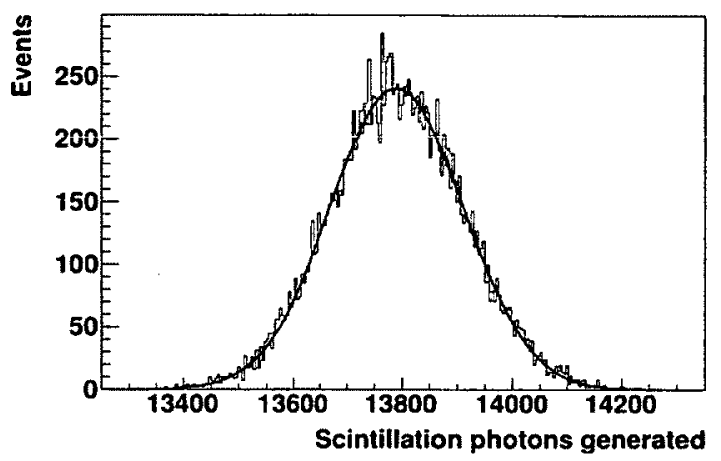

(b)

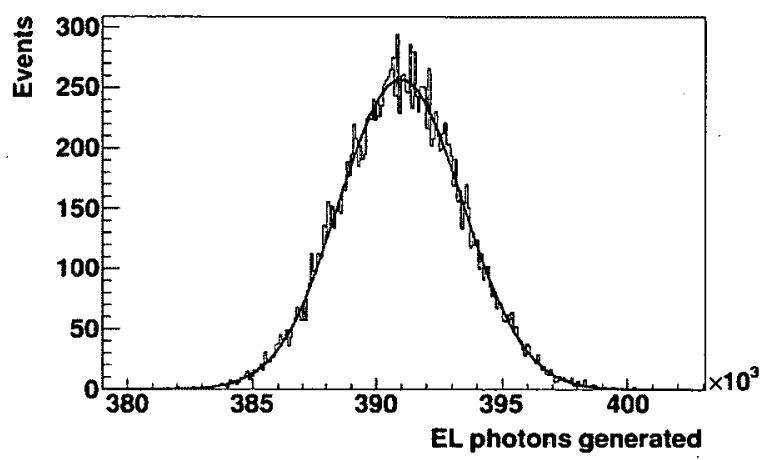

(d)

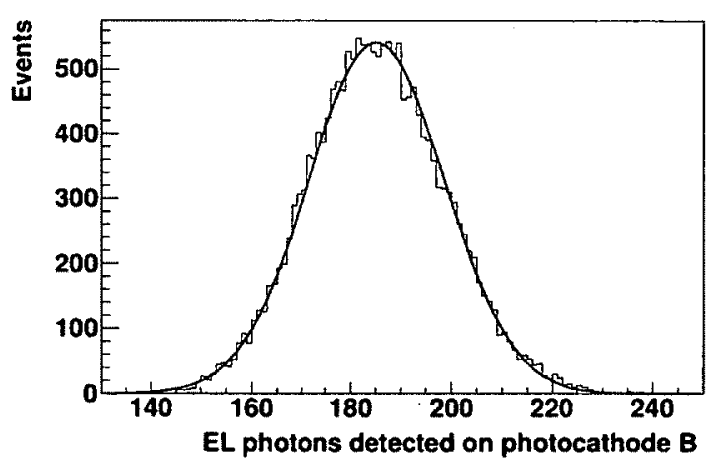

(f)

Figure 4.1: Distribution of the energy deposited in Xe (a), the numbers of scintillation photons (b), ionization electrons (c), and EL photons (d) generated, and the number of EL photons detected on the photocathodes on the EL region side (e) and on the opposite side (f) for 18526 electrons at $1 \mathrm{MeV}$ generated in $10 \mathrm{~atm} \mathrm{Xe}$. The parameters of the Gaussian fit are presented in Table 4.1. 


\begin{tabular}{lll} 
& Mean Value & Standard Deviation \\
\hline Energy deposited & $1000.02 \pm 0.02 \mathrm{keV}$ & $2.486 \pm 0.012 \mathrm{keV}$ \\
Scintillation Photons & $13789.5 \pm 0.9$ & $121.3 \pm 0.7$ \\
Ionization Electrons & $4546.1 \pm 0.2$ & $28.50 \pm 0.15$ \\
EL Photons Produced & $390963 \pm 18$ & $2451 \pm 13$ \\
EL Photons Detected on PC A & $3557.0 \pm 0.7$ & $83.6 \pm 0.4$ \\
EL Photons Detected on PC B & $185.03 \pm 0.10$ & $13.56 \pm 0.07$
\end{tabular}

Table 4.1: Fit parameters of the Gaussian fit for each of the plots of Figure 4.1

loses some. To test this hypothesis, the simulation was redone with the drift electric field set to zero, and the spectrum of energy deposited in xenon displayed a perfectly mono-energetic peak at $1 \mathrm{MeV}$.

The spectrum of the number of scintillation photons in Figure 4.1b displays a normal distribution with a standard deviation of approximately $\sqrt{N}$, which agrees with the parameters used in Geant4. For the number of ionization electrons produced, shown in Figure 4.1c, the fluctuation is dominated by the Fano factor. Since fluctuations in the number of EL photons emitted per electrons has not yet been implemented in the simulation, the variation in the total number of EL photons is driven by the variation in the number of electrons. Hence, the error in Figure 4.1d $\left(\sigma_{N} / \bar{N}\right)$ is equal to the one in Figure $4.1 \mathrm{c}$ as expected.

Finally, it should be noted that scaling down the number of ionization electrons and EL photons per electron affects the counting statistics in some of those distributions. Therefore, the standard deviations of the number of EL photons detected on the two photocathodes, shown in Figures 4.1e and 4.1f, do not reflect the resolution that should be achieved by the detector. The effect that those changes should have 
on the energy resolution can be understood through Equations 2.21 and 2.22, where $W_{i}$ would be multiplied by 10 and $n_{E L}$ divided by 50 .

\subsection{Detector Response Uniformity}

This section describes a study of the detection uniformity of electroluminescence photons emitted at different positions in the EL region. Individual electrons were generated $1 \mathrm{~mm}$ from the EL region so that their diffusion should be negligible. Since the TPC is radially symmetric, the electrons were created at 14 different locations along a straight. line in the $\mathrm{x}-\mathrm{y}$ plane from the origin to $(x, y)=(25.14,25.14) \mathrm{cm}$ at $1.796 \mathrm{~cm}$ intervals. This distance corresponds to half the diagonal length of the CsI pads. This way, half the positions were selected directly above the center of a pad, while the other half were directly above the corner of four pads, or in other words, over the intersections of the Teflon spacer grid between the fused silica window and the CsI layer. Hence, for the photocathode on the EL region side, the results should show the minimum and maximum shadowing effect caused by the spacer grid.

At each of these 14 positions, 10,000 electrons were generated. The simulation was made at a pressure of $10 \mathrm{~atm}$ and an electric field in the EL region of $70 \mathrm{kV}$ $\mathrm{cm}^{-1}$. However, the efficiency calculated this way should be independent of the pressure selected and the choice of pressure and electric field should only affect the EL yield, and hence the counting statistics. With the simple model of EL light production implemented, at this value of E/p, exactly $8624 \mathrm{EL}$ photons per electron were generated.

Figure 4.2 illustrates the distribution of the detected photons on the pads of the photocathode on the EL region side for electrons generated at four different positions: the two closest to the center and the two farthest ones. In every cases, the position of the ionization electrons is visible. This is important since it is these positions that will 
be used for the reconstruction of the trajectory of the tracks from incident charged particles.

Figure 4.3 shows this distribution for the photocathode on the opposite side of the TPC for electrons generated at the closest and farthest point from the center. The light distribution over the pads is seen to be relatively uniform.

For each electron simulated, the EL photon detection efficiency was calculated and averaged over the 10000 electrons generated at that position. The results are shown in Figure 4.4 with the error bars representing the standard deviation on the mean.

Figure 4.4a presents the results on the near side photocathode. The differences in detection efficiency between light emitted over the center of a pad and over the corner of four pads are clearly seen by the two levels of data points (the points around $1.01 \%$ and those around $0.81 \%$ ). The efficiency is $20 \%$ lower when an electron reaches the EL region over the intersection of the spacer grid then when it is located over the center of a pad. Hence, the variation of efficiency for single electrons on the near side photocathode is clearly dominated by the effect of the spacer grid. However, this fluctuation is for EL light emitted from single electrons, while for the detection of tracks, the efficiency of the light detection will be averaged out over the area where the track is located. The lower efficiency at $r=23.3 \mathrm{~cm}$ is likely due to the proximity of the edge of the photocathode, which is only about $1.4 \mathrm{~cm}$ away.

Figure $4.4 \mathrm{~b}$ shows the results for the far side photocathode. The average efficiency is seen decreasing as the EL light is produced away from the center, passing from $0.047 \%$ at the center to $0.043 \%$ near the edge. This represents an efficiency $8.4 \%$ lower near the edge than at the center.

Hence, the distribution of light on the far side photocathode is quite uniform, with a soft dependence on the radial position. Adding to the simulation a reflectivity to the copper rings cage should further soften this dependence. Furthermore, since 


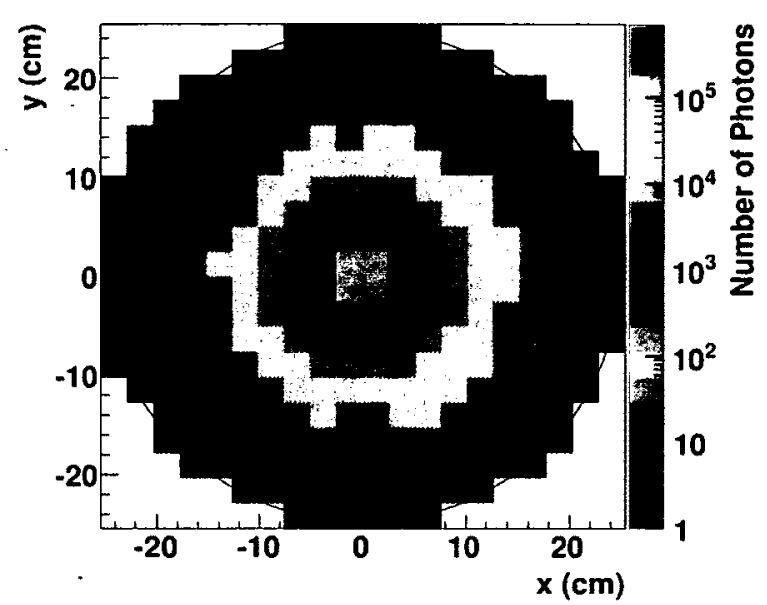

(a)

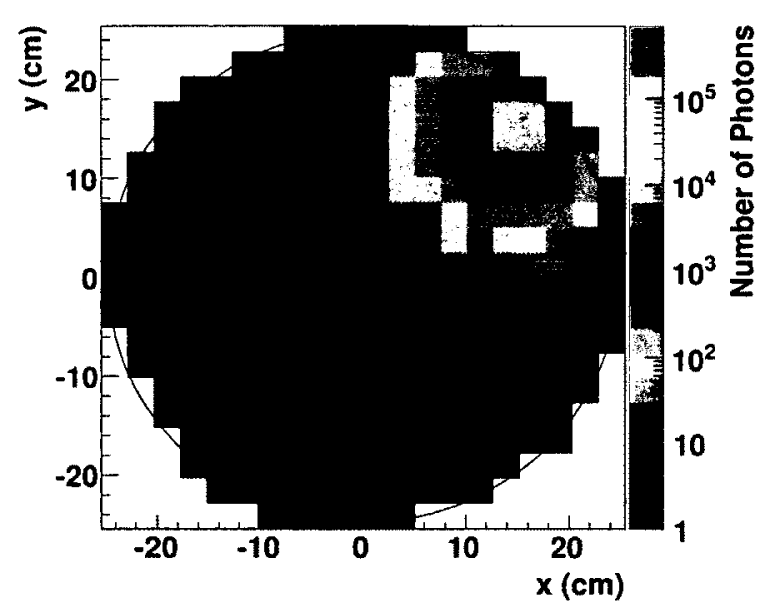

(c)

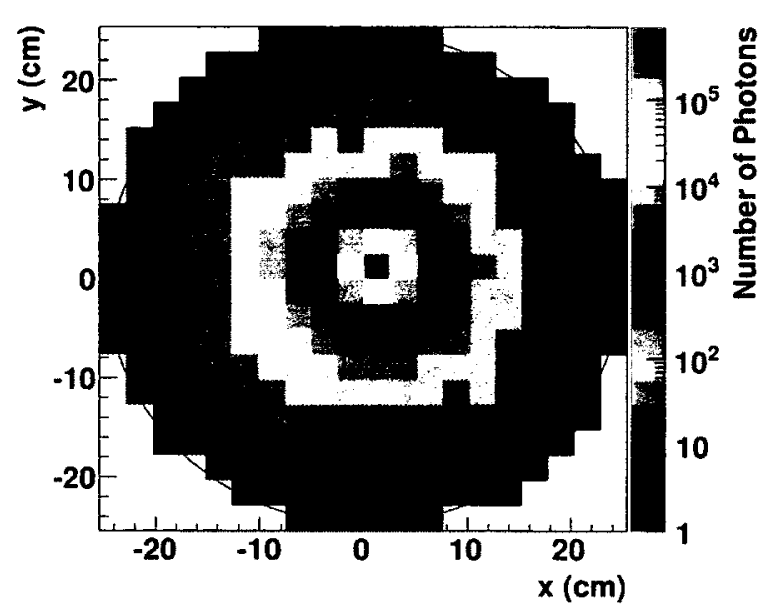

(b)

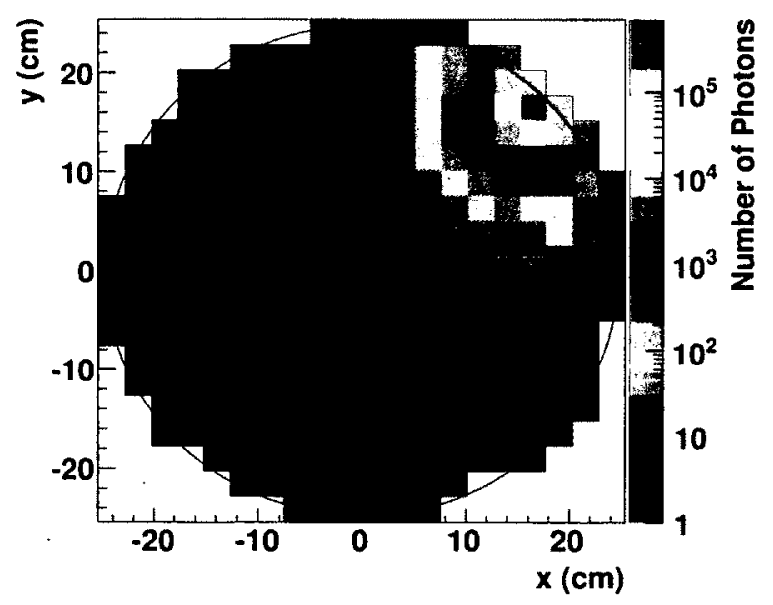

(d)

Figure 4.2: Distribution on the photocathode on the EL region side of the detected EL photons from 10000 electrons generated at different locations on the $\mathrm{x}-\mathrm{y}$ plane. In (b) and (d), the light was produced directly over the center of a CsI pad while in (a) and (c) it was produced over the Teflon spacer grid. 


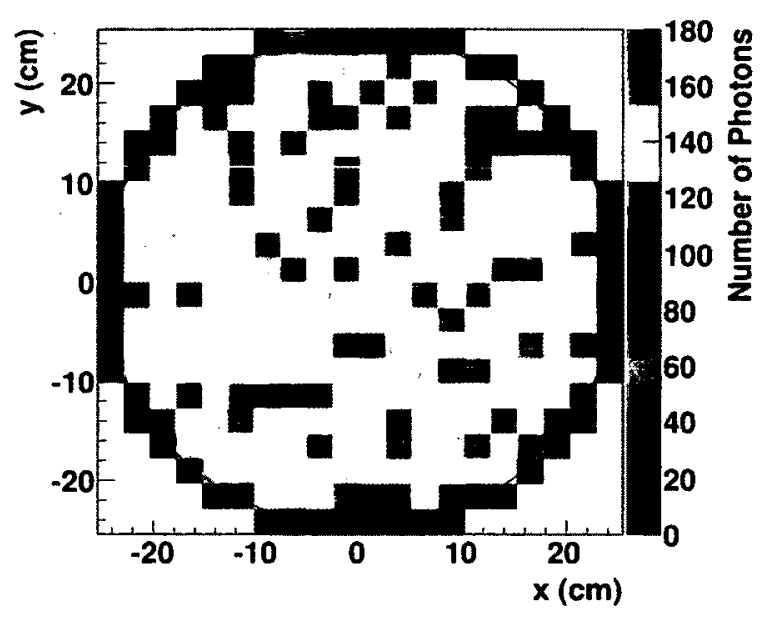

(a)

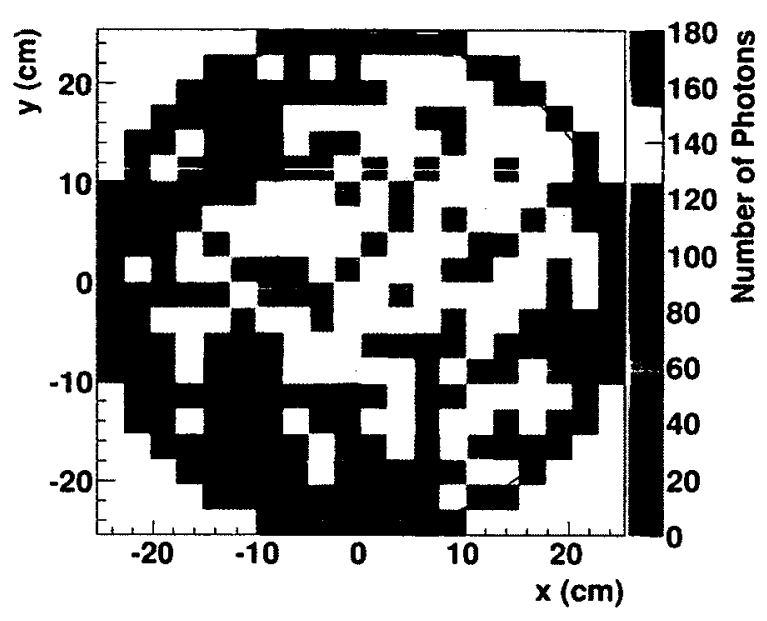

(b)

Figure 4.3: Distribution on the photocathode at the far end of the TPC of the detected EL photons from 10000 electrons generated at different locations on the $x-y$ plane. In (a), the photons were emitted at the origin (as in Figure 4.2a), and in (b) at $\mathrm{x}=\mathrm{y}=16.51 \mathrm{~cm}$ (as in Figure $4.2 \mathrm{~d}$ ).

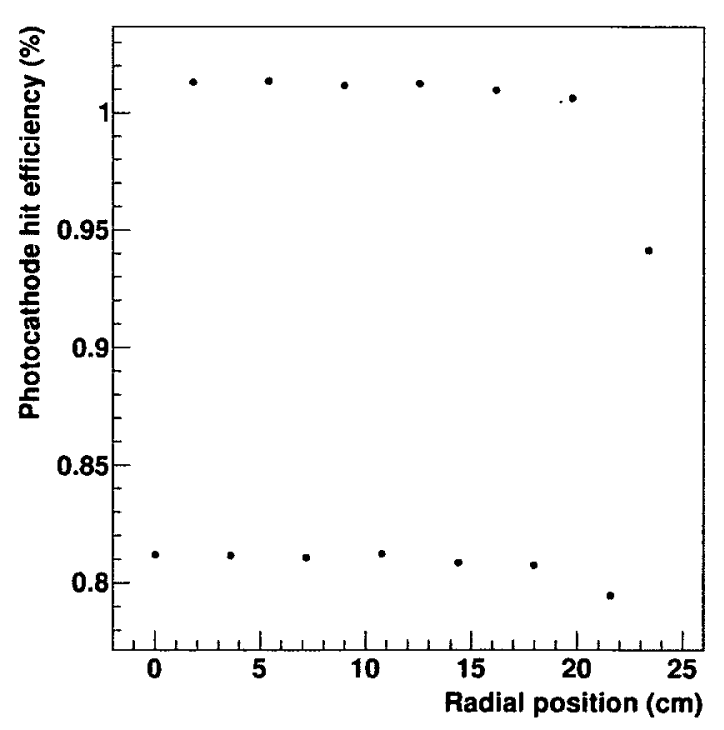

(a) Photocathode on the anode side (near the EL region).

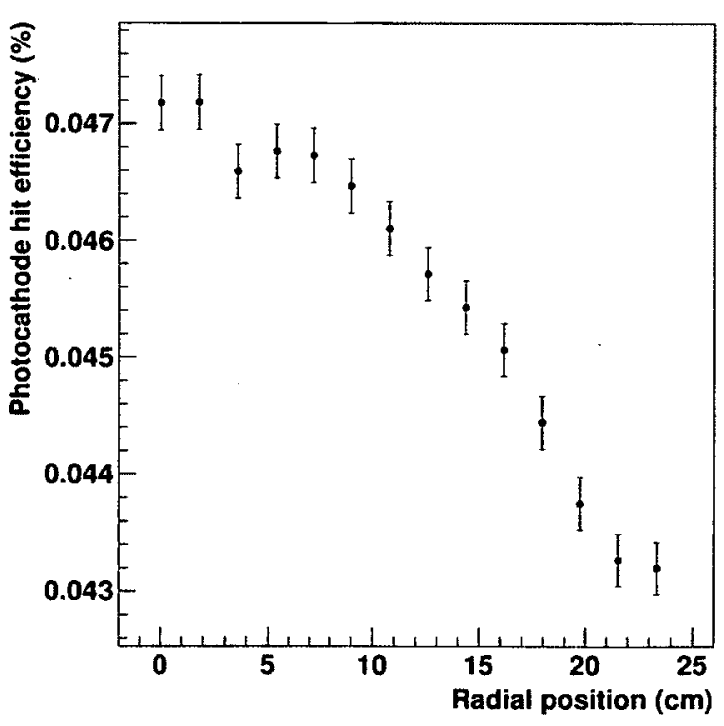

(b) Photocathode on the cathode side.

Figure 4.4: Detection efficiency of each photocathode for EL photons originating at various radial positions. 
the light is distributed on every pads, the effect of the variation in efficiency between each pad is attenuated. Therefore, measuring the energy from the light detected on the far side photocathode might offer the best energy resolution.

\subsection{Natural Gamma Background Simulation}

If two or more ionizing events occur simultaneously in the detector, their signals will combine on the readout in a single pulse. This phenomenon is known as pile-up. In the XEP TPC, because of the long charge collection time (i.e., the drift time of the electrons across the vessel), the signals will form slowly. During this time interval, events from other origins, such as the natural background radiations or cosmic rays, can form in the detector and produce pile-up.

Radioactive isotopes will naturally be found in the detector construction materials and the surrounding environment. The most important isotopes to consider are ${ }^{238} \mathrm{U}$, ${ }^{232} \mathrm{Th}$, and ${ }^{40} \mathrm{~K}$, which should be most abundant in the concrete, as well as ${ }^{222} \mathrm{Rn}$ present in the air. ${ }^{40} \mathrm{~K}$ decays directly to a stable element, while the other three trigger a chain of decays of short lived isotopes, some of them emitting gamma rays at specific energies. To investigate the effect of these sources of gamma radiation, the Monte Carlo simulation was used to estimate the rate and spectrum of energy deposited in the detector from ${ }^{238} \mathrm{U},{ }^{232} \mathrm{Th}$, and ${ }^{40} \mathrm{~K}$.

The concrete walls, floor and ceiling were assumed to be the main sources of radioactive isotopes around the detector. To estimate the isotope concentration in these volumes, measurements of the gamma radioactivity in the assembly area, where the XEP experiment is being built, were made with a high-purity germanium (HPGe) detector. For comparison, values were also taken from "Radiological protection principles concerning the natural radioactivity of building materials" (1999) by the Directorate-General Environment, Nuclear Safety and Civil Protection of the 
European Commission [67]. This document reported specific activities (decay rate per $\mathrm{kg}$ of concrete) of $40 \mathrm{~Bq} \mathrm{~kg}-1,30 \mathrm{~Bq} \mathrm{~kg}^{-1}$, and $400 \mathrm{~Bq} \mathrm{~kg}{ }^{-1}$ for ${ }^{238} \mathrm{U},{ }^{232} \mathrm{Th}$, and ${ }^{40} \mathrm{~K}$, respectively.

\subsubsection{Germanium detector measurement}

Prior to the simulation, a Ge detector was used to measure the gamma background where the detector was going to be assembled. A. Ge detector is a type of semiconductor detector used for gamma spectroscopy. It contains a high purity germanium volume in which gamma rays from external sources deposit their energy mainly through Compton scattering. In doing so, they create pairs of free electrons and holes which drift under an electric field towards the electrodes where they produce a pulse that can be amplified and measured.

With the gain applied by the amplifier, the spectrum was measured within a range of $108 \mathrm{keV}$ to $3416 \mathrm{keV}$. The energy calibration of the readout channels was done with weak gamma sources of various isotopes, namely ${ }^{133} \mathrm{Ba}(356.02 \mathrm{keV}),{ }^{22} \mathrm{Na}$ $(1274.53 \mathrm{keV}),{ }^{60} \mathrm{Co}(1173.24 \mathrm{keV}$ and $1332.50 \mathrm{keV})$, and ${ }^{137} \mathrm{Cs}(661.66 \mathrm{keV})$. It should be noted that those sources cover only the first half of the energy range measured, so the energy calibration can be expected to be inaccurate in the higher end of the spectrum. The resulting spectrum, measured over a period of 70.2 hours, is presented in Figure 4.5 .

An estimation of the photopeak detection efficiency was obtained with the same gamma sources as the ones used for the energy calibration. By placing these sources at a distance $d$ from the Ge detector and knowing the dimensions of the active $\mathrm{Ge}$ volume, the rate of gamma rays directed toward the detector was estimated as

$$
R_{\gamma}=a b \frac{\delta A}{4 \pi d^{2}}
$$




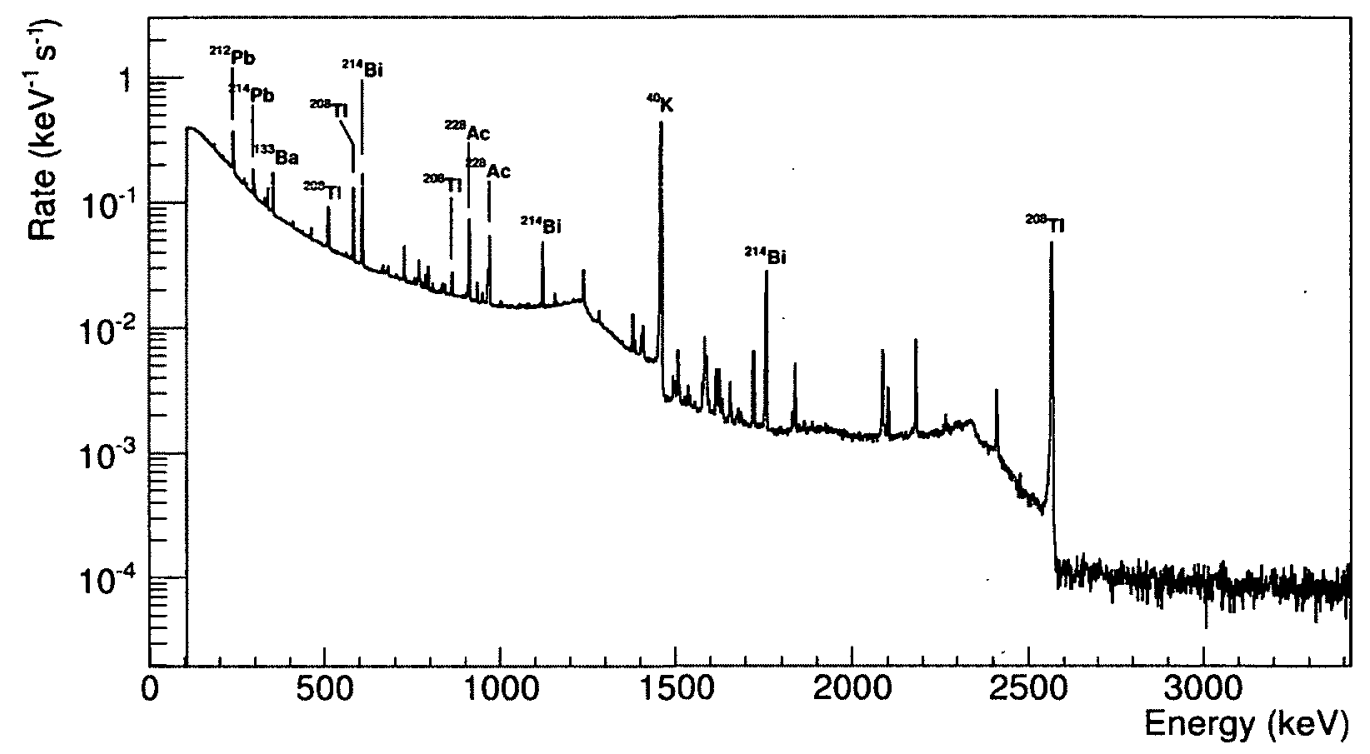

Figure 4.5: Spectrum of the natural gamma ray background measured in the assembly area with a Ge detector. The isotope origin of some of the most prominent lines are identified.

where $a$ is the activity of the source, $b$ is the branching ratio of the observed gammas, and $\delta A$ is the area of the detector active volume seen from the source position. The detection efficiency was calculated by dividing the measured count rate by the estimated total rate.

To obtain the number of counts in the photopeaks, the continuous Compton spectrum had to be subtracted from the total counts in the corresponding channels. This was done by fitting the peaks with a Gaussian plus a third order polynomial, which represents the continuous part of the spectrum and was used to find the values to subtract from the total counts in these channels. An example of the fit of a photopeak is shown in Figure 4.6. The plot of these efficiencies as a function of the different isotopes energies, shown in Figure 4.7, was fitted to obtain a curve of the photopeak detection efficiency as a function of energy. 


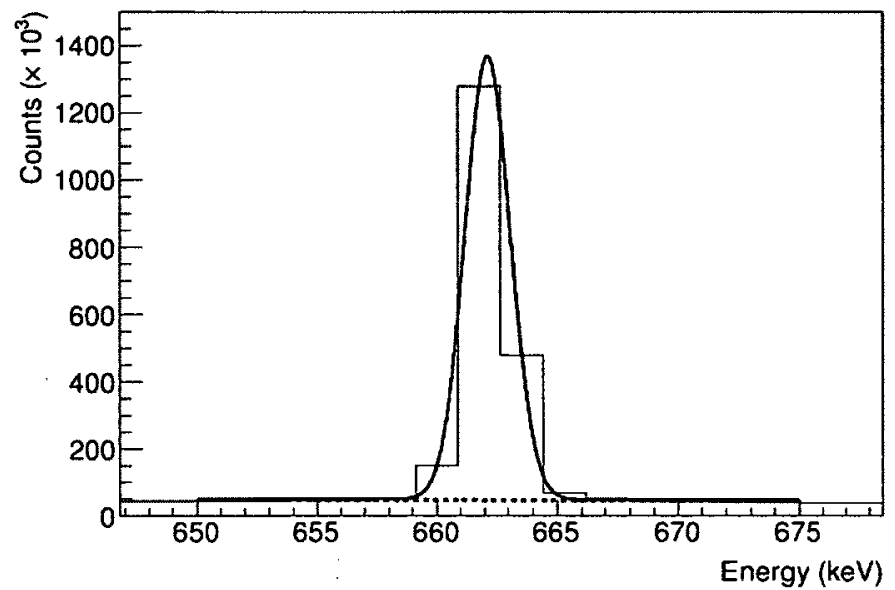

Figure 4.6: Example of fit for the extraction of the number of counts in a photopeak from a ${ }^{137} \mathrm{Cs}$ source. The fit function is a Gaussian plus a third order polynomial, shown in dashed line, which is used to estimate the background counts.

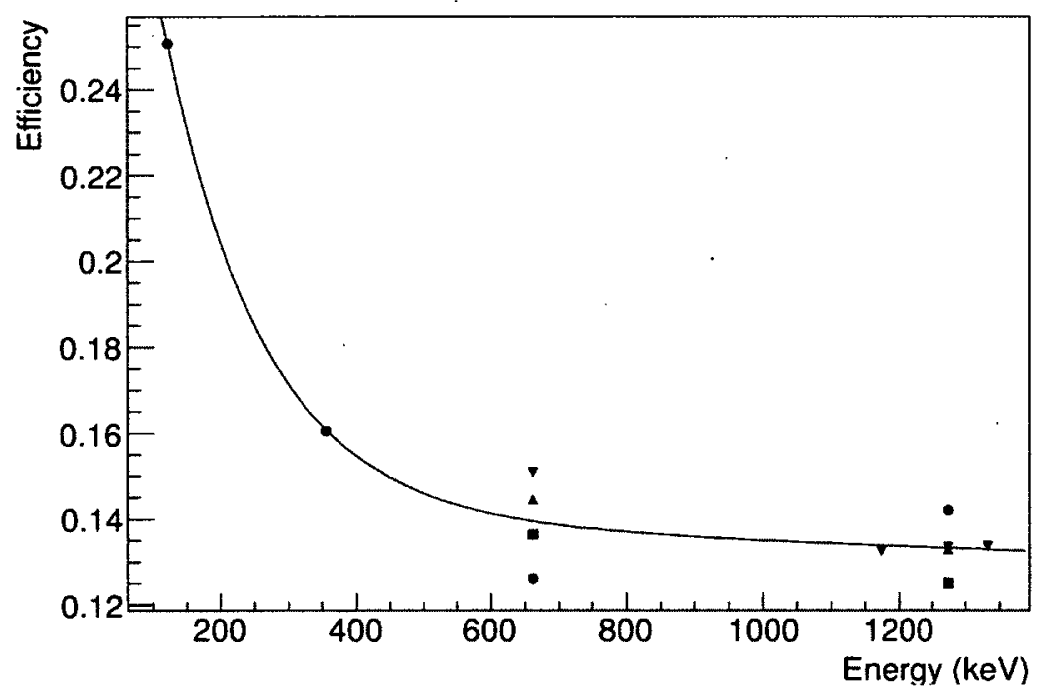

Figure 4.7: Fit of the measured efficiencies of the Ge detector. The different sets of points correspond to different runs.

The number of counts in the photopeaks of some of the main lines of the background spectrum was calculated in the same way, and the detection efficiency curve was used to estimate the gamma ray flux at those energies. The resulting values are presented in Table 4.2 . 


\begin{tabular}{lccc}
\hline \hline Isotope & Energy $(\mathrm{keV})$ & Rate $\left(\mathrm{s}^{-1}\right)$ & Activity $\left(\mathrm{s}^{-1} \mathrm{~kg}^{-1}\right)$ \\
\hline \hline${ }^{40} \mathrm{~K}$ & 1460.82 & 1.463 & 147 \\
\hline${ }^{212} \mathrm{~Pb}$ & 238.63 & 0.475 & 17.4 \\
\hline${ }^{214} \mathrm{Bi}$ & 609.32 & 0.346 & 11.0 \\
& 1120.29 & 0.0974 & 7.7 \\
& 1764.49 & 0.0919 & 6.0 \\
\hline${ }^{208} \mathrm{Tl}$ & 583.19 & 0.233 & 11.1 \\
& 860.56 & 0.0282 & 8.1 \\
& 2614.51 & 0.1858 & 4.6 \\
\hline${ }^{228} \mathrm{Ac}$ & 338.32 & 0.0913 & 12.9 \\
& 911.2 & 0.1829 & 9.0 \\
& 968.97 & 0.1454 & 11.0 \\
\hline${ }^{214} \mathrm{~Pb}$ & 295.22 & 0.1644 & 14.3 \\
\hline \hline
\end{tabular}

Table 4.2: Gamma detection rate for gamma rays of various isotopes energies measured in the assembly area with a Ge detector. The last column shows the activity of the parent isotope in the concrete calculated by the method explained bellow.

From these numbers, a crude estimate of the specific activity of ${ }^{238} \mathrm{U},{ }^{232} \mathrm{Th}$, and ${ }^{40} \mathrm{~K}$ in the concrete of the assembly area was calculated. To avoid the complexity of taking into consideration the geometry of the room, the detector was assumed to be in the center of a spherical concrete shell of inner radius $R$. Let $a$ be the activity per unit mass of the parent isotope (i.e., ${ }^{238} \mathrm{U},{ }^{232} \mathrm{Th}$, or ${ }^{40} \mathrm{~K}$ ), $b$ be the branching ratio between the parent and one of its daughter isotopes (e.g, ${ }^{212} \mathrm{~Pb},{ }^{214} \mathrm{Bi},{ }^{208} \mathrm{Tl}$, etc.), and $I$ be the intensity for the emission of gamma at a specific energy $E$ (i.e., the probability of the corresponding radiation to be emitted when the daughter isotope decays). Then, the gamma emission rate at this energy from a volume component $d V$ of the concrete with density $\rho$ will be equal to $a b I \rho d V$. If $d V$ is located at a 
distance $r$ within the wall and $\delta A$ is the cross section area of the active volume of the detector, the measured hit rate should be

$$
R_{h i t}=a b I \rho d V \frac{\delta A}{4 \pi(R+r)^{2}} e^{-\mu r} \epsilon(E)
$$

where $\epsilon(E)$ is the efficiency of the detector at this energy and $\mu$ is the attenuation coefficient of concrete. With $d V=(R+r)^{2} \sin \theta d r d \theta d \phi$ and integrating over the whole volume, the hit rate simply becomes $R_{h i t}=a b I \rho \delta A\left(1-e^{-\mu l}\right) \epsilon(E) / \mu$, where $l$ is the thickness of the wall. Then, the parent isotope activity per unit mass presents the concrete can be estimated as

$$
a=\frac{R_{h i t} \mu}{b I \rho \delta A\left(1-e^{-\mu l}\right) \epsilon(E)}
$$

Taking the values of $\mu$ from the National Institute of Standards and Technology (NIST) tables of X-ray mass attenuation coefficients [68], the intensities $I$ from the National Nuclear Data Center (NNDC) Chart of Nuclides [69], and the branching ratios $b$ from the Argonne National Laboratory [70], $a$ was calculated for the energies listed in Table 4.2 and the average was taken for each the three parent isotopes. The results are $9.7 \mathrm{~Bq} \mathrm{~kg}^{-1}$ for ${ }^{238} \mathrm{U}, 10.6 \mathrm{~Bq} \mathrm{~kg}^{-1}$ for ${ }^{232} \mathrm{Th}$, and $147 \mathrm{~Bq} \mathrm{~kg}^{-1}$ for ${ }^{40} \mathrm{~K}$.

The uncertainties on those estimates are probably large due to the difficulties in calibrating the detector and the simplification of the room geometry in the calculations. However, based on those numbers, it can be assumed that the activity in the concrete is not likely to be significantly larger than the ones reported in the "Radiological protection principles concerning the natural radioactivity of building materials" (1999) quoted above. In the next section, these two set of estimates will be considered. 


\subsubsection{The Simulation Setup and Results}

The objective of this simulation was to estimate the rate of background ionizing events in the detector originating from the concrete of the assembly area. Since such a simulation is computationally very intensive, it was performed in two steps. The first one consisted in estimating the flux of gamma rays, including their energy and direction, at the surface of the concrete walls, floor and ceiling, for a given isotope chain: The second step consisted in simulating the interactions within the detector of the gamma rays escaping the concrete surfaces, generating them using the angular distribution and energy spectrum from the first step. Dividing the task in these two steps allowed to reduce considerably the time spent by the program to calculate the interaction of the gamma rays with the concrete volume.

The Radioactive Decay Module (RDM) presented in Section 3.1.1 was used to generate the particles produced by the decay of ${ }^{40} \mathrm{~K}$ and the isotopes in the ${ }^{238} \mathrm{U}$ and

${ }^{232} \mathrm{Th}$ decay chains. The isotopes of those two chains were assumed to be in secular equilibrium, meaning that they all decay at the same rate as they are produced, and hence have the same decay rate. The parent nuclides were generated in a concrete volume $12 \mathrm{~m}$ by $12 \mathrm{~m}$ and $30.48 \mathrm{~cm}$ thick. This thickness correspond to the thickness of the south wall of the assembly area. For each of the three parent isotopes, 600,000 decays were simulated and the gamma rays reaching a $10 \mathrm{~m}$ by $10 \mathrm{~m}$ area centred over one of the surfaces of the wall were recorded. Both the energy and the direction at which the photons exited the wall were kept.

The average time $t$ that would be required for $N_{\text {decay }}$ decays to occur in a concrete volume of mass $M$ is given by

$$
t=\frac{N_{\text {decay }}}{a M}
$$


where $a$ is the activity concentration $(\mathrm{Bq} / \mathrm{kg})$ for this isotope. Then, if $N_{\text {decay }}$ is the number of parent isotope decays generated and $N_{\gamma}$ represents the number of gamma rays detected on the $10 \mathrm{~m}$ by $10 \mathrm{~m}$ area, the flux at the concrete's surface can be calculated as

$$
f=\frac{N_{\gamma} a M}{N_{\text {decay }} A}
$$

where $A=100 \mathrm{~m}^{2}$.

Assuming a concrete density of $2300 \mathrm{~kg} \mathrm{~m}^{-3}$, the simulated mass was $M=100,950 \mathrm{~kg}$ and the calculated fluxes were $13,138 \mathrm{~m}^{-2} \mathrm{~s}^{-1}, 8964 \mathrm{~m}^{-2} \mathrm{~s}^{-1}$, and $5835 \mathrm{~m}^{-2} \mathrm{~s}^{-1}$ for ${ }^{238} \mathrm{U},{ }^{232} \mathrm{Th}$, and ${ }^{40} \mathrm{~K}$, respectively.

The flux should be dependent on the thickness of the concrete wall. To measure the importance of this parameter, the first step of the simulation was repeated for ${ }^{238} \mathrm{U}$ with a wall thickness of $15.24 \mathrm{~cm}$, which represents the thickness of the south wall and the ceiling of the assembly area. The resulting flux was $11,807.6 \mathrm{~m}^{-2} \mathrm{~s}^{-1}$, which is $10 \%$ lower than with the thicker wall of 30.48 .

The second step consisted of the simulation of the interaction of the radiation with the detector. The gamma rays were generated along the position of the two walls closest to the detector as well as the floor and the ceiling. The distances between the detector and these four surfaces were chosen according to the dimensions shown in Figure 3.2. The south and west walls were considered over a length of $10 \mathrm{~m}$ and $9 \mathrm{~m}$, respectively, which delimited the dimension of the floor and ceiling (both $9 \mathrm{~m}$ by $10 \mathrm{~m}$ ). This provides a total wall area of $444.24 \mathrm{~m}^{2}$. The position of the vertex of each gammas was randomly chosen uniformly along these surfaces and the direction and energy were selected from the distributions calculated in the first step, using them as probability density functions.

For each of the three decay chains, the spectrum of number of counts detected as 
a function of the energy deposited was calculated both with and without the water boxes and steel plates shielding shown in Figure 3.2. To obtain the spectra of count rate, all bins of the spectra were multiplied by a factor

$$
t^{-1}=\frac{A f}{N_{g e n}} .
$$

where $N_{g e n}$ is the number of gamma rays generated, $A$ is the total concrete surface considered and $f$ is the flux calculated in the first step. For each of the three isotopes considered, $8 \times 10^{8}$ events were generated. The resulting spectra with $10 \mathrm{~atm} \mathrm{Xe}$ are presented in Figures 4.8 to 4.10 , where the activities in Equation 4.5 were taken from [67]. Table 4.3 and 4.4 shows the total count rates integrated over all energies for the TPC filled with 10 atm and 2 atm Xe, respectively. 


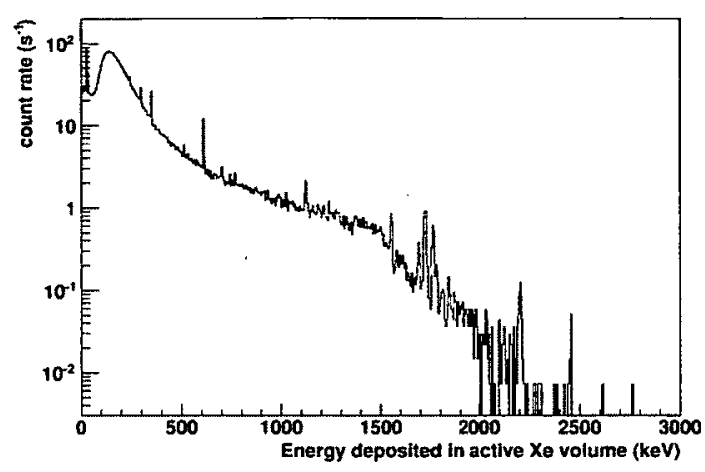

(a) Without Shielding

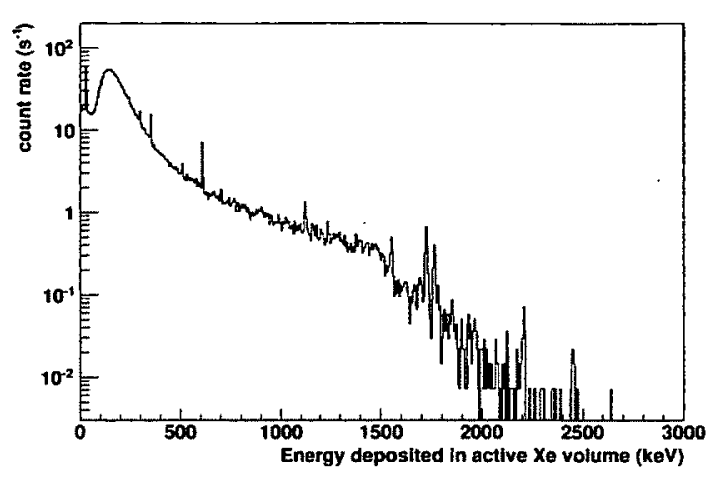

(b) With Shielding

Figure 4.8: Simulated spectrum from ${ }^{238} \mathrm{U}$ with $10 \mathrm{~atm}$.Xe.

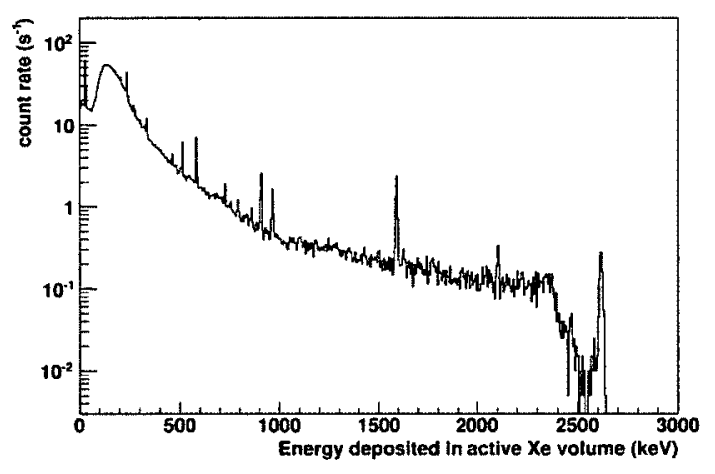

(a) Without Shielding

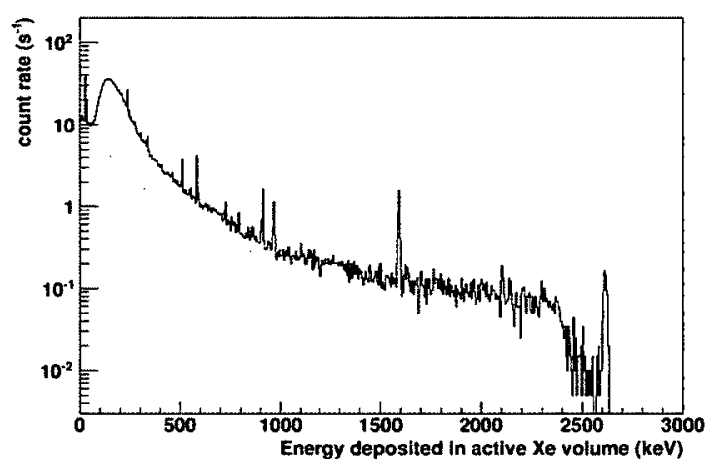

(b) With Shielding

Figure 4.9: Simulated spectrum from ${ }^{232} \mathrm{Th}$ with $10 \mathrm{~atm}$ Xe.

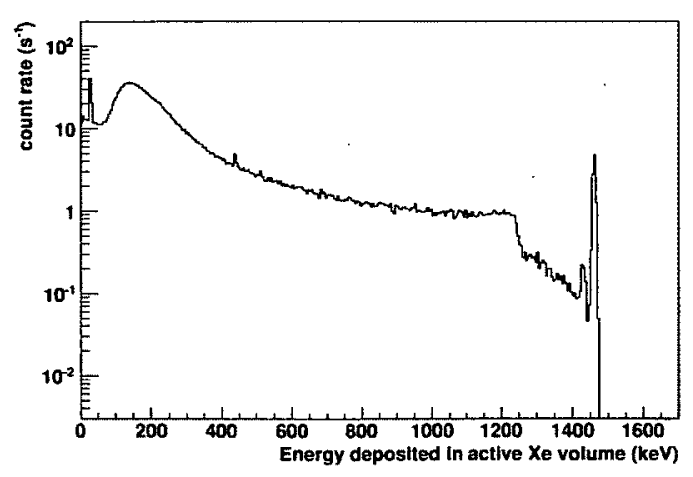

(a) Without Shielding

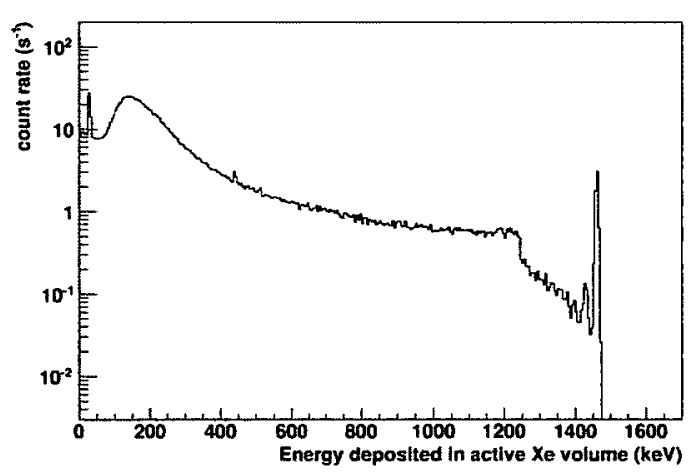

(b) With Shielding

Figure 4.10: Simulated spectrum from ${ }^{40} \mathrm{~K}$ with 10 atm Xe. 


\begin{tabular}{lcccc} 
Parent Isotope & ${ }^{238} \mathrm{U}$ & ${ }^{232} \mathrm{Th}$ & ${ }^{40} \mathrm{~K}$ & ${ }^{238} \mathrm{U}$ (with thin walls) \\
\hline \hline Activity (Bq/kg) & 40 & 30 & 400 & 40 \\
\hline Rate Without Shielding $\left(\mathrm{s}^{-1}\right)$ & 3448 & 2247 & 1651 & 3099 \\
Rate With Shielding $\left(\mathrm{s}^{-1}\right)$ & 2286 & 1468 & 1124 & 2054 \\
\hline \hline Activity (Bq/kg) & 9.7 & 10.6 & 147 & 9.7 \\
\hline Rate Without Shielding $\left(\mathrm{s}^{-1}\right)$ & 839 & 797 & 605 & 754 \\
Rate With Shielding $\left(\mathrm{s}^{-1}\right)$ & 556 & 521 & 412 & 500
\end{tabular}

Table 4.3: Simulation results for the detection rate of gamma rays from ${ }^{40} \mathrm{~K}$ and the decay chains of ${ }^{238} \mathrm{U}$ and ${ }^{232} \mathrm{Th}$ in the concrete of the assembly area with $10 \mathrm{~atm}$ xenon. The last column represents the count rate for of gamma rays originating from thinner walls $(15.24 \mathrm{~cm}$ instead of $30.48 \mathrm{~cm})$.

\begin{tabular}{lccc} 
Parent Isotope & ${ }^{238} \mathrm{U}$ & ${ }^{232} \mathrm{Th}$ & ${ }^{40} \mathrm{~K}$ \\
\hline \hline Activity $(\mathrm{Bq} / \mathrm{kg})$ & 40 & 30 & 400 \\
\hline Rate Without Shielding $\left(\mathrm{s}^{-1}\right)$ & 1430 & 958 & 658 \\
Rate With Shielding $\left(\mathrm{s}^{-1}\right)$ & 955 & 628 & 456 \\
\hline \hline Activity (Bq/kg) & 9.7 & 10.6 & 147 \\
\hline Rate Without Shielding $\left(\mathrm{s}^{-1}\right)$ & 348 & 340 & 241 \\
Rate With Shielding $\left(\mathrm{s}^{-1}\right)$ & 232 & 223 & 167
\end{tabular}

Table 4.4: Simulation results for the detection rate of gamma rays from ${ }^{40} \mathrm{~K}$ and the decay chains of ${ }^{238} \mathrm{U}$ and ${ }^{232} \mathrm{Th}$ in the concrete of the assembly area with 2 atm xenon. 


\subsubsection{Cosmic Ray Background}

In addition to the natural gamma rays from the environment, cosmic rays will contribute to the external background. Muons are the most abundant charged particles of cosmogenic origin at ground level and their flux has often been measured over the past decades. In Reference [71], the flux of muons at ground level was calculated based on the parametrization of experimental measurements. The rate of muons with an energy greater than $0.2 \mathrm{GeV}$ across a horizontal surface is reported as $R_{H}=172.32 \mathrm{~m}^{-2} \mathrm{~s}^{-1}$ and across a vertical surface as $R_{V}=56.5 \mathrm{~m}^{-2} \mathrm{~s}^{-1}$. With a cylindrical active volume of length $L=84 \mathrm{~cm}$ and diameter $D=49.5 \mathrm{~cm}$, the total rate from muons in the $\mathrm{XEP}$ detector is estimated as $R_{\mu} \approx D L R_{H}+2\left(D L+\pi D^{2} / 4\right) R_{V}=142 \mathrm{~s}^{-1}$.

\subsubsection{Pile-up Estimation}

If one or more events occur in the detector in rapid succession, their signals will combine and only the sum of the two signals will be observed. This phenomenon, known as pile-up, will be one of the main effects of the background on the performance of the detector. A higher pile-up ratio in the measured tracks will make it more challenging to identify the signals.

In the XEP experiment, the time it will take for a signal to form in the TPC will be determined by the drift time of the ionization electrons. If the active volume of the TPC has a length of $84 \mathrm{~cm}$ and the drift velocity is taken as $1.1 \times 10^{5} \mathrm{~cm} / \mathrm{s}$, the electrons will take at most $t=0.76 \mathrm{~ms}$ to drift to the anode. A simple way to calculate an upper limit on the pile-up probability is to consider the probability that one or more background particles leave a signal in the detector during a time window $t$ before or after the measured events, that is, during the time interval $\left[t_{0}-t, t_{0}+t\right]$, where $t_{0}$ is the time at which the measured event occurs. However, most tracks in the TPC will be contained within a limited volume and the time window can be reduced 
based on the spatial separation of the tracks. For example, if one tries to detect $1 \mathrm{MeV}$ electrons at 10 atm, most tracks should be contained within a length of $6 \mathrm{~cm}$, giving a time window of $t=(6 \mathrm{~cm}) /\left(1.1 \times 10^{5} \mathrm{~cm} \mathrm{~s}^{-1}\right)=54.5 \mu \mathrm{s}$.

If these events are independent from the background hits, the probability that at least one background particle leaves a signal in the TPC during this time window is given by summing the Poisson distributions for all numbers of events $n$ greater than zero:

$$
P(n>0)=\sum_{n=1}^{\infty} \frac{\lambda^{n}}{n !} e^{-\lambda}=1-e^{-\lambda}
$$

where $\lambda=2 t r$ and $r$ is the background detection rate. Here, $2 t$ represents the length of the time interval $\left[t_{0}-t, t_{0}+t\right]$. Summing the rates with shielding from Table 4.3 together with the muon rate calculated above gives two possible rates of 5020 and 1630 counts per second. With those rates, the probabilities that an event will pile-up with one or more background hits are $42 \%$ and $16 \%$, respectively. Note that the pileup probability can be reduced by also taking into account the spatial distribution of the tracks in the $\mathrm{x}-\mathrm{y}$ plane.

The results obtained suggest that, with the current gamma ray shielding installed, the detected background rates should be high, resulting in a significant pile-up probability. Therefore, increasing the gamma ray shielding and constructing a muon veto counter would be beneficial.

\subsection{Radioactive sources simulation}

To help determine the strength of the gamma sources that should be used for the calibration of the detector, the response of the TPC to various radioactive sources was simulated. The primary particles were generated as mono-energetic point sources of 
various energies at two different positions on top of the detector steel vessel illustrated in Figure 4.11. The first position was over the center of the active Xe area, while the second one was $24 \mathrm{~cm}$ from the position of the drift electric field cathode on the side opposite to the EL region. The sources chosen in this study, with the energies of their simulated gamma rays, were ${ }^{133} \mathrm{Ba}(356.0 \mathrm{keV}),{ }^{137} \mathrm{Cs}(661.7 \mathrm{keV}),{ }^{60} \mathrm{Co}(1173.2 \mathrm{keV}$ and $1332.5 \mathrm{keV})$, and ${ }^{22} \mathrm{Na}(511.0 \mathrm{keV}$ and $1274.5 \mathrm{keV})$.

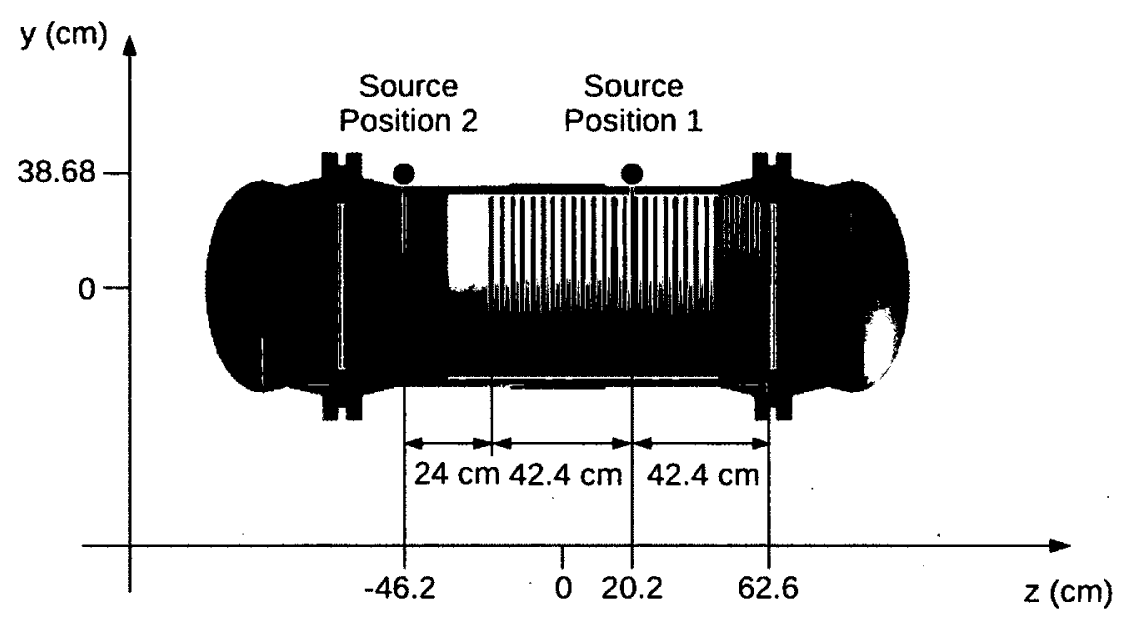

Figure 4.11: Position of the simulated gamma sources.

Figures 4.12 to 4.15 show the spectra of the energy deposition rate in the Xe active volume for the four sources simulated at an arbitrary source activity. In Figures 4.14 and 4.15 , the results of the two gamma energies simulated for ${ }^{60} \mathrm{Co}$ and ${ }^{22} \mathrm{Na}$ were combined in a single spectrum. Figure 4.16 shows the spatial distribution of the energy deposited in the detector for the ${ }^{137} \mathrm{Cs}$ gamma rays at $661.7 \mathrm{keV}$.

For each energy spectra produced, the number of counts in the photopeak was used to find the peak efficiency. The total efficiency, which measures the ratio of generated gamma rays that deposited any energy in the TPC, was also calculated. The results are presented in Table 4.5 for the sources located over the center of the Xe active volume (position 1) and in Table 4.6 for the sources $24 \mathrm{~cm}$ from the cathode (position 2). 


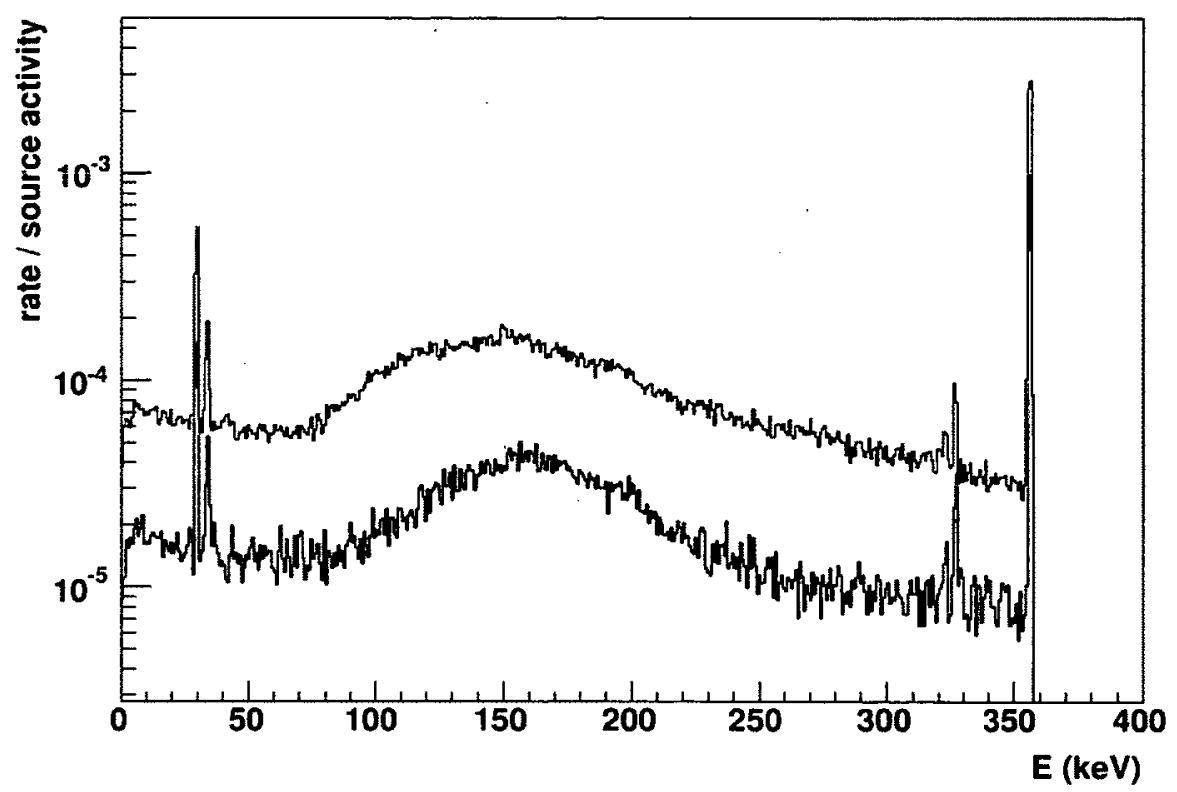

Figure 4.12: Spectra of the energy deposited by the $356 \mathrm{keV}$ gamma rays from a ${ }^{133} \mathrm{Ba}$ source. The higher and lower spectra correspond to the source located at position 1 and 2 respectively.

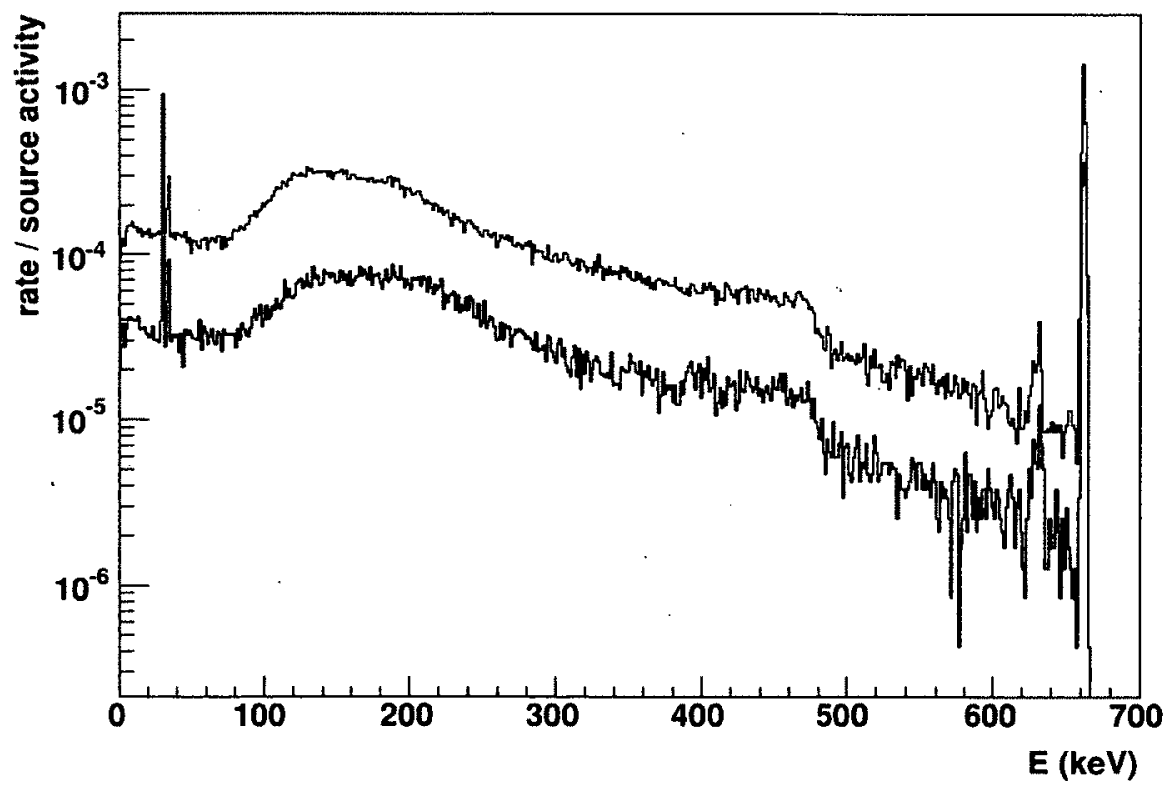

Figure 4.13: Spectra of the energy deposited by the $661.7 \mathrm{keV}$ gammas from a ${ }^{137} \mathrm{Cs}$ source. The higher and lower lines correspond to position 1 and 2 respectively. 


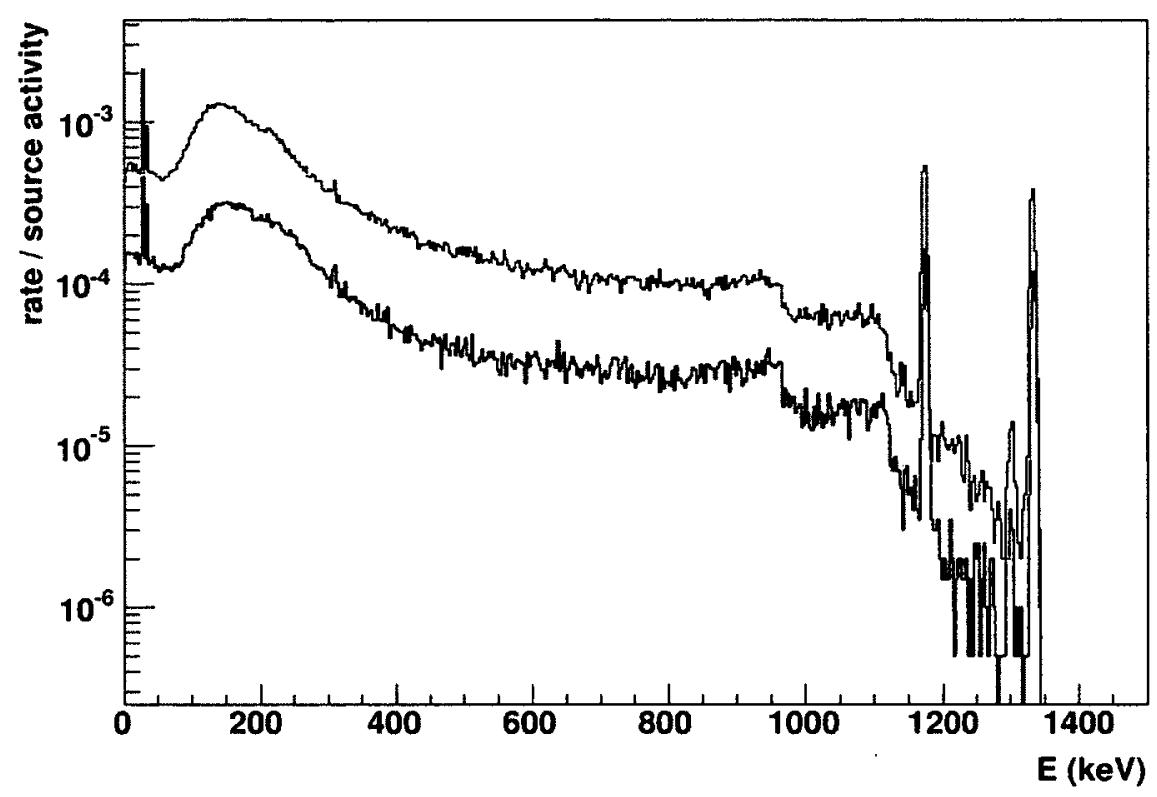

Figure 4.14: Spectra of the energy deposited by the $1173.2 \mathrm{keV}$ and $1332.5 \mathrm{keV}$ gamma rays from a ${ }^{60} \mathrm{Co}$ source located at position 1 (higher line) and position 2 (lower line).

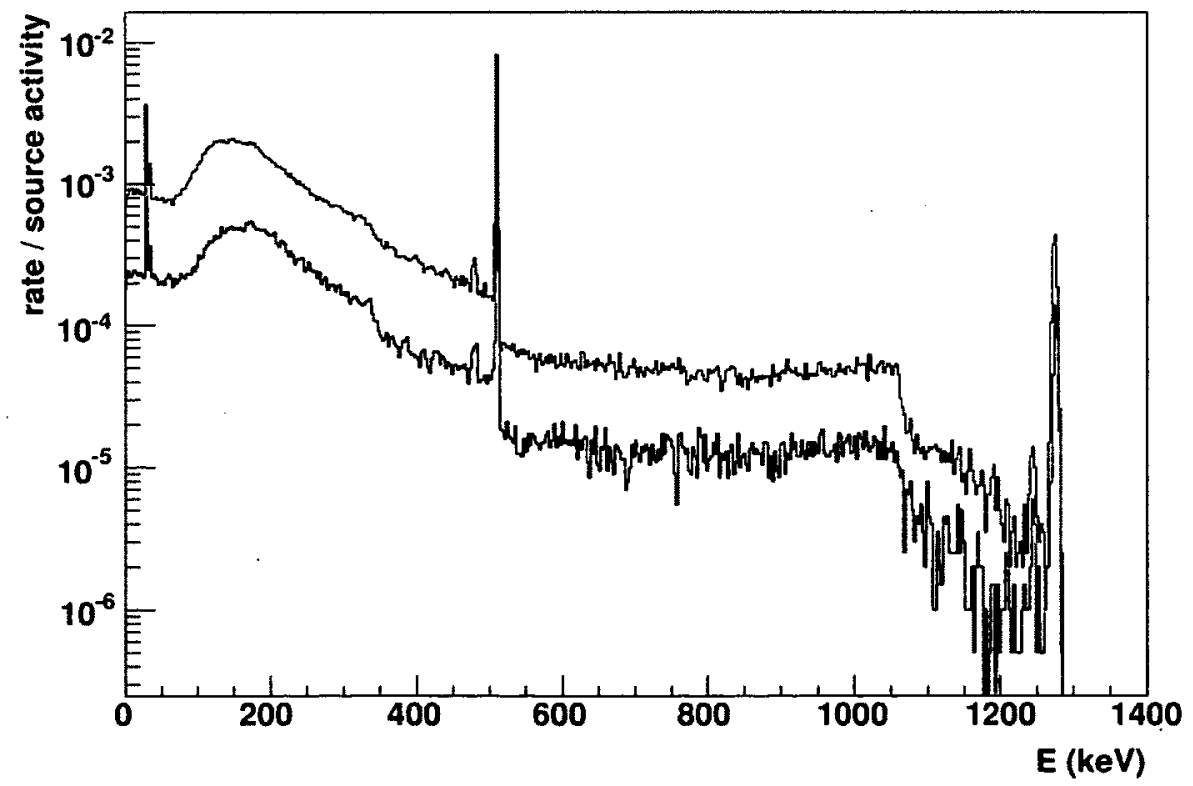

Figure 4.15: Spectra of the energy deposited by the $51.0 \mathrm{keV}$ and $1274.5 \mathrm{keV}$ gammas from a ${ }^{22} \mathrm{Na}$ source located at position 1 (higher line) and 2 (lower line). 


\begin{tabular}{ccccccc} 
& & \multicolumn{2}{c}{ Peak efficiency } & & \multicolumn{2}{c}{ Total efficiency } \\
\cline { 3 - 4 } Isotope & Energy & 2 atm & 10 atm & & 2 atm & 10 atm \\
\hline${ }^{133} \mathrm{Ba}$ & $356.0 \mathrm{keV}$ & 0.00119 & 0.00897 & 0.0259 & 0.0691 \\
${ }^{22} \mathrm{Na}$ & $511.0 \mathrm{keV}$ & 0.000510 & 0.00491 & 0.0242 & 0.0671 \\
${ }^{137} \mathrm{Cs}$ & $661.7 \mathrm{keV}$ & 0.000253 & 0.00308 & 0.0229 & 0.0649 \\
${ }^{60} \mathrm{Co}$ & $1173.2 \mathrm{keV}$ & 0.000039 & 0.00125 & 0.0210 & 0.0595 \\
${ }^{22} \mathrm{Na}$ & $1274.5 \mathrm{keV}$ & 0.000029 & 0.00115 & 0.0208 & 0.0590 \\
${ }^{60} \mathrm{Co}$ & $1332.5 \mathrm{keV}$ & 0.000024 & 0.00101 & 0.0207 & 0.0581
\end{tabular}

Table 4.5: Photopeak and total detection efficiency for gamma sources located over the center of the Xe active area.

\begin{tabular}{cccccccc} 
& & \multicolumn{2}{c}{ Peak efficiency } & & \multicolumn{2}{c}{ Total efficiency } \\
\cline { 3 - 4 } Isotope & Energy & 2 atm & $10 \mathrm{~atm}$ & & $2 \mathrm{~atm}$ & $10 \mathrm{~atm}$ \\
\hline${ }^{133} \mathrm{Ba}$ & $356.0 \mathrm{keV}$ & 0.000368 & 0.00244 & & 0.00838 & 0.0164 \\
${ }^{22} \mathrm{Na}$ & $511.0 \mathrm{keV}$ & 0.000145 & 0.00136 & & 0.00779 & 0.0170 \\
${ }^{137} \mathrm{Cs}$ & $661.7 \mathrm{keV}$ & 0.000074 & 0.00085 & & 0.00732 & 0.0169 \\
${ }^{60} \mathrm{Co}$ & $1173.2 \mathrm{keV}$ & 0.000010 & 0.00031 & & 0.00656 & 0.0160 \\
${ }^{22} \mathrm{Na}$ & $1274.5 \mathrm{keV}$ & 0.000007 & 0.00029 & & 0.00649 & 0.0156 \\
${ }^{60} \mathrm{Co}$ & $1332.5 \mathrm{keV}$ & 0.000006 & 0.00027 & & 0.00649 & 0.0156
\end{tabular}

Table 4.6: Photopeak and total detection efficiency for gamma sources located $24 \mathrm{~cm}$ from the position of the drift field cathode. 


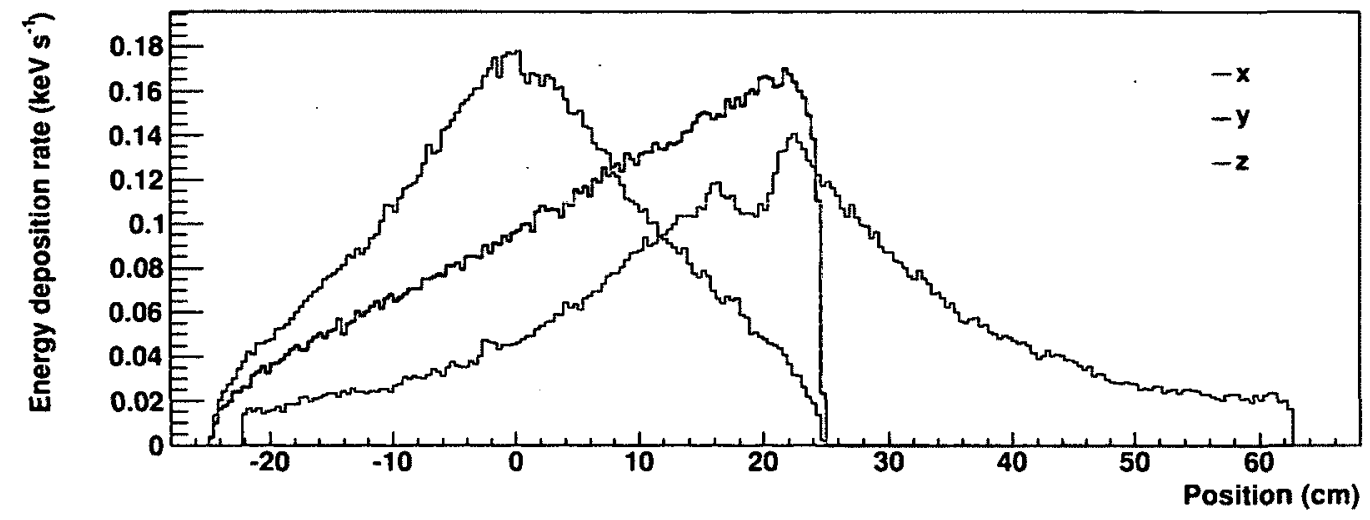

(a) Position 1

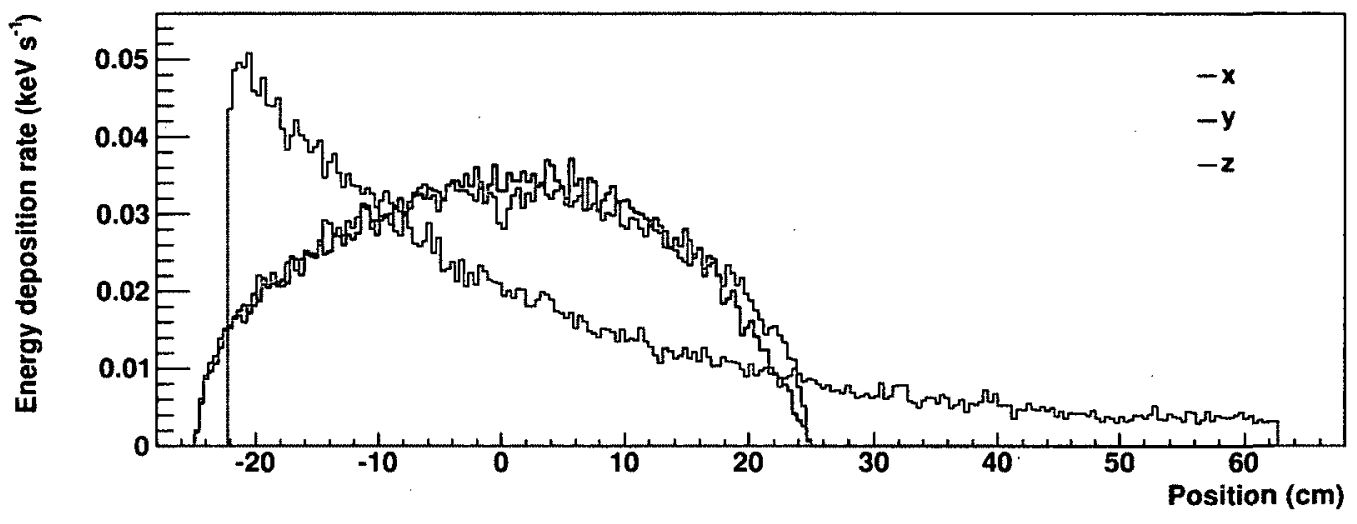

(b) Position 2

Figure 4.16: Spatial distribution of the energy deposition rate in the active $\mathrm{Xe}$ from a $661.7 \mathrm{keV}$ mono-energetic gamma source located at position $1(\mathrm{x}=0 \mathrm{~cm}$, $y=30.68 \mathrm{~cm}, \mathrm{z}=20.2 \mathrm{~cm})$ and $2(\mathrm{x}=0 \mathrm{~cm}, \mathrm{y}=30.68 \mathrm{~cm}, \mathrm{z}=-46.2 \mathrm{~cm})$. The region with a lower energy deposition just below the center of the $z$ axis in (a) is due to the presence of one of the copper rings at $\mathrm{z}=19.8 \mathrm{~cm}$.

When selecting the activity of the sources to be used for calibrating the detector, pile-up will be an important preoccupation. A larger count rate allows us to accumulate a more statistically significant sample of hits more rapidly and improves the signal to background ratio. However, increasing the count rate also increases the probability of event pile-up. Therefore, a source with an activity too high can degrade the results by producing too much dead time. 
Assuming that the time sequence of the hits in the detector follows a Poisson distribution, every time a hit occurs in the photopeaks, the probability that no other gamma ray is detected during a time $t$ before and after the hit is

$$
P(n=0)=\frac{\lambda^{0}}{0 !} e^{-\lambda}=e^{-\lambda}
$$

where $\lambda=2 t r$ and $r$ is the hit rate. If $a$ is the source activity, $b$ is the branching ratio of gamma emission at the photopeak energy, and $\epsilon(E)$ is the total detection efficiency of the detector at this energy, the count rate is related to the source activity as $r=a b \epsilon(E)$. If we want the calibration data set to have a minimum fraction $\beta$ of events without pile-up, we need $P(n=0) \geq \beta$, which gives an upper limit on the gamma source activity of

$$
a \leq-\frac{\ln \beta}{2 t b \epsilon(E)}
$$

If more than one gamma energy is considered for the same isotope, $r$ in Equation 4.8 should be replaced by the total detection rate $\sum_{i} r_{i}$, where the $r_{i}$ 's are the detection rates for the different gamma energies. This results in a limit on the source activity of

$$
a \leq-\frac{\ln \beta}{2 t \sum_{i}\left(b_{i} \epsilon\left(E_{i}\right)\right)}
$$

where $b_{i}$ and $E_{i}$ are the branching ratios and energies of the different gamma rays emitted. The results of this calculation for the four sources considered are presented in Table 4.7 where the time window $t$ was taken again as $(6 \mathrm{~cm}) /\left(1.1 \times 10^{5} \mathrm{~cm} \mathrm{~s}^{-1}\right)=$ $54.5 \mu$ s as in Section 4.3 . 


\begin{tabular}{rrr} 
Isotope & Limit on Source Activity \\
\hline${ }^{133} \mathrm{Ba}$ & $22600 \mathrm{~Bq}$ & $0.610 \mu \mathrm{Ci}$ \\
${ }^{137} \mathrm{Cs}$ & $17500 \mathrm{~Bq}$ & $0.473 \mu \mathrm{Ci}$ \\
${ }^{60} \mathrm{Co}$ & $8230 \mathrm{~Bq}$ & $0.222 \mu \mathrm{Ci}$ \\
${ }^{22} \mathrm{Na}$ & $5360 \mathrm{~Bq}$ & $0.145 \mu \mathrm{Ci}$
\end{tabular}

Table 4.7: Maximum gamma source activity, given in $\mathrm{Bq}$ and $\mu \mathrm{Ci}$, for different isotopes in order to have a single rate pile-up ratio lower than 0.9 . A charge collection time window of $54.5 \mu \mathrm{s}$ and a Xe pressure of 10 atm were assumed.

Of course, the pile-up calculated above will combine with the effect from the natural background radiation discussed in Section 4.3. Considering the probability of pile-up with background events estimated in that section, a total ratio of events without pile-up of 0.9 is likely unachievable with the shielding currently in place around the detector.

In conclusion, this investigation of the effect of gamma ray sources on the detector will facilitate choosing the source strengths to obtain for the calibration of the TPC as well as the locations for placing them around the detector. 


\section{Chapter 5}

\section{Neural Network}

The development of artificial neural networks have first been motivated by the study of biological nervous systems and the entirely different way they compute information compared to conventional digital computers. There seems to be no universally accepted definition of what a neural network exactly is, but it usually involves a number of interconnected simple computing nodes called "neurons" or "processing units". One possible definition is offered by Haykin (1994) [72] who adapted it from Aleksander and Morton (1990) [73]:

A neural network is a massively parallel distributed processor that has a natural propensity for storing experiential knowledge and making it available for use. It resembles the brain in two respects:

1. Knowledge is acquired by the neural network through a learning process.

2. Inter-neuron connection strengths known as synaptic weights are used to store knowledge.

Hence, a neural network is an adaptive system. The learning process mentioned in this definition involves the ability of the network to alter its internal structure based on external or internal information until it can produce the desired calculation. Neural 
networks are usually implemented using electrical components or simulated with a digital computer and they are often used to model complicated relationships and to find patterns in data.

The application of artificial neural networks has found wide interest among particle physicists. These systems have already proved useful in common feature recognition problems such as off-line data analysis and low level experimental triggers.

As mentioned in Section 2.3.2, distinguishing between signals resulting from double beta decay events and those resulting from the various sources of background will be both challenging and essential. The topology of the tracks in the detector should present certain features that could help to make this distinction. However, the tracks will have complicated shapes due to the scattering of the particles in the gas.

The aim of this study is to test the capability of a certain type of neural network, called a multilayer perceptron (MLP), to participate in the task of background rejection in XEP. The performance of the algorithm was tested, using the implementation of the ROOT program's class of MLP, to distinguish between different classes of events, such as ones formed by one electron versus others formed by two electrons, or as double track events, where the two tracks are either joint (like in $\beta \beta(0 \nu)$ ) or disjoint (like in background pile-up). Furthermore, its performance for identifying the point of origin of a track in xenon was also investigated. Before presenting the results, the MLP algorithm will be explained in the next section, while Section 5.2 presents the ROOT's class of MLP used in this project.

\subsection{Multilayer Perceptron}

The type of neural network used in this study is called a multilayer perceptron (MLP). An example of the structure of the MLP is shown in Figure 5.1. It consists of successive layers of "neurons", or "nodes", interconnected with each neuron of the adjacent 


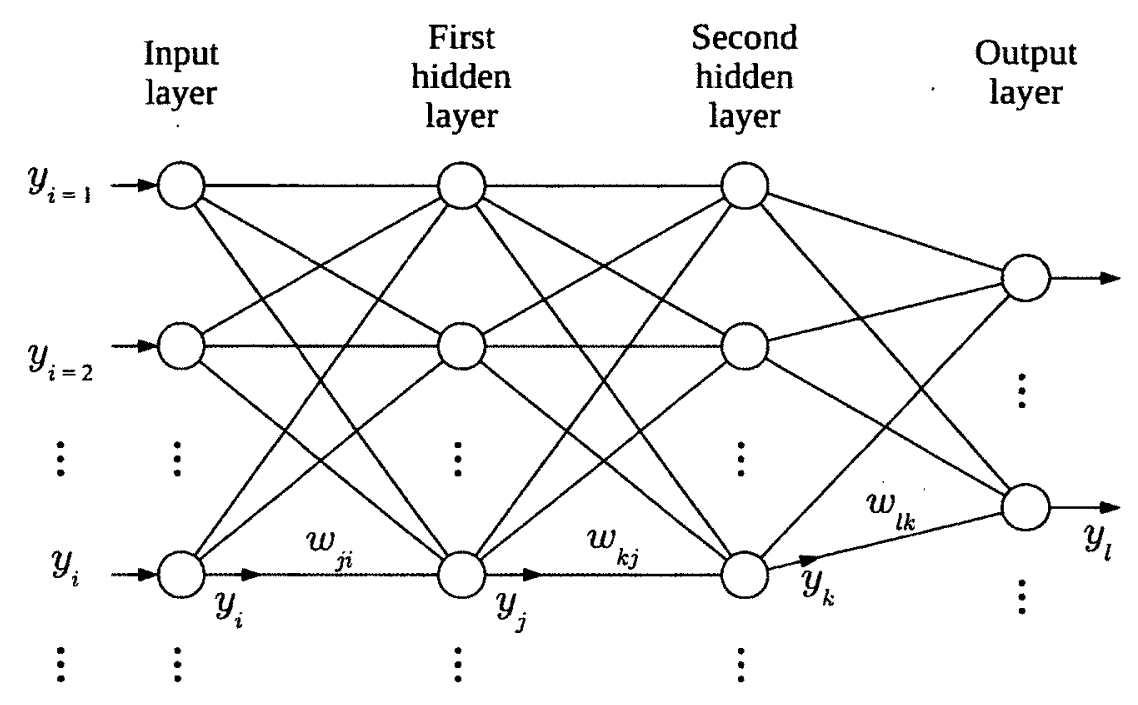

Figure 5.1: An example of a multilayer perceptron neural network.

layers. At the front end of the network is the "input layer", which consists of sensory nodes that simply pass the input information forward to the next layer. It is followed by one or more "hidden layers" of computational units, which process the information through a nonlinear activation function. Finally, the network ends with the "output layer", also made of computational nodes. The connections between the neurons are called "synapses" and carry a weight.

Hence, a MLP is a type of feedforward network: the input signal propagates through the network layer by layer. It works with a supervised learning technique called error back-propagation, which adjusts the synaptic weights of each synapse until the output of the network becomes adequately close to the desired outcome. Hence, it is necessary for the training process to provide a set of input data with the corresponding desired output values.

\subsubsection{Feedforward propagation}

To understand how the MLP works, let consider a neural network like the example illustrated in Figure 5.1. To compute an output, each input neuron must be provided 
with one of the components $y_{i}$ of the input vector. Next, the neurons of the first hidden layer compute a weighted linear combination of the input values and add to it a bias $\theta_{j}$, giving

$$
a_{j}=\sum_{i=1}^{N_{i n}} w_{j i} y_{i}+\theta_{j}
$$

where $w_{j i}$ are the synaptic weights between the input layer and the first hidden layer and $N_{i n}$ is the number of input neurons. By adding an extra input variable $y_{0}$, whose value is permanently set to $y_{0}=1$, the bias can be written as $w_{j 0}$ and the previous equation can be formulated in a more compact form:

$$
a_{j}=\sum_{i=0}^{N_{i n}} w_{j i} y_{i}
$$

The result of this linear sum is then transformed by the activation function to produce the output of this neuron:

$$
y_{j}=f\left(a_{j}\right)
$$

In each successive layer, the output of each neuron is calculated in the same way. If the first hidden layer has $N_{1}$ units, each neuron of the second hidden layer constructs a weighted linear combination given by

$$
a_{k}=\sum_{j=0}^{N_{1}} w_{k j} y_{j}
$$

Then, the neuron's output is calculated again by mapping this summation with the activation function: 


$$
y_{k}=f\left(a_{k}\right)
$$

This calculation is repeated for each neuron of the network, one layer at a time, until the output neurons produce the output of the network.

An important criteria that the activation function has to meet is to be smooth (i.e., differentiable everywhere), for reasons that will become clear in the next section. The logistic sigmoid function, plotted in Figure 5.2a and defined as

$$
f(y) \equiv \frac{1}{1+e^{-y}}
$$

meets this criteria and is ofter used in MLP. Another function commonly employed is the hyperbolic tangent plotted in Figure $5.2 \mathrm{~b}$ and given by:

$$
f(y) \equiv \tanh (y) \equiv \frac{1-e^{-2 y}}{1+e^{-2 y}}
$$

Finally, the output layer is commonly implemented with a linear activation function. The reason for this is that the use of sigmoid units would limit the range of

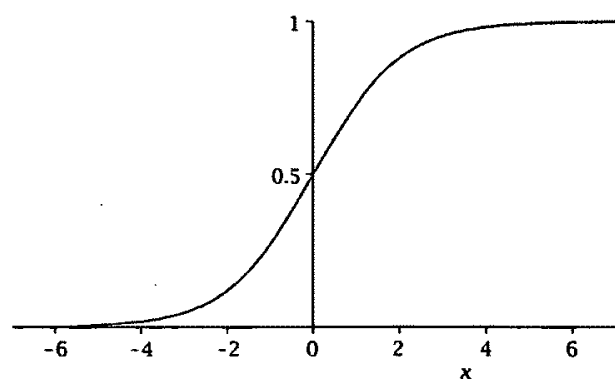

(a) Sigmoid function

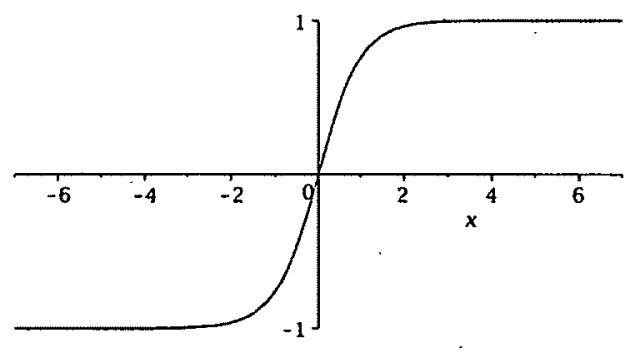

(b) Tanh function

Figure 5.2: Sigmoid and Tanh functions. 
possible output values to the range of the sigmoid function, which could be undesirable in some cases.

\subsubsection{Error back-propagation}

The training process of the MLP consists in adjusting the synaptic weights in order to make the outputs as close as possible to the targeted value. For this, the neural network needs to be presented with a series of input vectors, or patterns, together with the associated targets. The network's calculated outputs and the desired targets are compared with a suitable error function, which is minimized with respect to the weights. A possible error function which is commonly used is the sum of the difference squared between the outputs $y_{l}$ and the targets $t_{l}$ divided by two [72,74]:

$$
E^{(n)}=\frac{1}{2} \sum_{l=1}^{N_{\text {out }}}\left(y_{\ell}-t_{l}\right)^{2}
$$

Here, the superscript $n$ labels the particular input vectors and $N_{\text {out }}$ is the number of output neurons. Most training algorithms involve an iterative process where a different input vector is presented at every iteration to find the weight adjustment that will minimize the error.

The problem lies in identifying which weights can be regarded as responsible for generating the error. A solution to this involves evaluating the derivative of the error function with respect to each of the weights in the network. If the error is chosen as a differentiable function of the network output, such as the one of Equation 5.8 and the activation function is also differentiable, then it can be seen from Equations 5.2, 5.8 , and 5.3 that the error is also a differentiable function of all the weights. The algorithm to evaluate these derivatives is called error back-propagation. Once these derivatives are found, a variety of optimization algorithms exist to adjust the weights 
in order to reduce or minimize the error.

To understand how error back-propagation works, first note that the derivatives of the error with respect to the weight of the synapses that connect to the output layer are straightforward to evaluate. Let consider again a MLP like the one illustrated in Figure 5.1, where $l$ labels the different units of the output layer. It can be shown that

$$
\frac{\partial E^{(n)}}{\partial w_{l k}}=\delta_{l} y_{k}
$$

where $y_{k}$ is the output of the $k$ 's neuron of the last hidden layer and

$$
\delta_{l} \equiv f^{\prime}\left(a_{l}\right) \frac{\partial E^{(n)}}{\partial y_{l}}
$$

where $f^{\prime}\left(a_{l}\right)$ is the derivative of the activation function evaluated at $a_{l}$.

For the weights connecting the last hidden layer from the preceding one, the derivatives can be shown to be [74]

$$
\frac{\partial E^{(n)}}{\partial w_{k j}}=\delta_{k} y_{j}
$$

with

$$
\delta_{k} \equiv f^{\prime}\left(a_{k}\right) \sum_{l} w_{l k} \delta_{l}
$$

Therefore, the $\delta$ 's for the output units must be first evaluated from Equation 5.10, after which Equation 5.12 can be applied recursively to the weights of every other units, layer by layer, starting from the end of the network. 


\subsubsection{Weights Adjustment}

The error back propagation algorithm offers an efficient technique to obtain the derivatives of the error function with respect to the synaptic weights. These derivatives must then be used to find new values for the weights that would minimize the error. This is a multi-variable function optimization problem. Several solutions to such problems exist from the mathematical theory of unconstrained minimization and many of them can be directly applied to neural networks.

The situation can be visualized more easily by grouping all the weights of the network in a vector $\mathbf{w}$ and viewing the error function $E(\mathbf{w})$ as an error surface in weight space. The problem now consists in finding the global minimum of $E(\mathbf{w})$ using the gradient $\nabla E(\mathbf{w})$ obtained with error back-propagation. Since the error function is generally non-linear, a closed form solution for the minimum is generally impossible to calculate. Instead, the different algorithms used for this involve different ways of updating the weight vector in a succession of iteration steps of the form

$$
\mathbf{w}^{(t+1)}=\mathbf{w}^{(t)}+\Delta \mathbf{w}^{(t)}
$$

where $t$ label the iteration step.

During the training process, an "epoch" is defined as an iteration over all the input vectors (or patterns) of the training set. The weights adjustment $\Delta \mathbf{w}^{(t)}$ can be applied either after each pattern has been presented, using the error $E^{(n)}$ computed for the individual patterns, or at the end of each epoch using the average error

$$
E=\frac{1}{N} \sum_{n=1}^{N} E^{(n)}
$$

where $N$ is the number of patterns in the training set. The former method is sometime 
called "online" or "pattern-by-pattern" learning, while the later one is called "batch" learning.

One of the simplest of these algorithms is called gradient descent and consists of incrementing $\mathbf{w}$ in the direction opposite to the gradient $\nabla E\left(\mathbf{w}^{(t)}\right)$. To increase the learning rate and to avoid instability (i.e., oscillation of $\mathbf{w}$ ), a momentum term is often added, and the weight increment becomes

$$
\Delta \mathbf{w}^{(t)}=-\eta \nabla E\left(\mathbf{w}^{(t)}\right)+\mu \Delta \mathbf{w}^{(t-1)},
$$

where $\eta$ and $\mu$ are called the learning rate and the momentum constant, respectively.

An alternative approach to gradient descent consists again of moving the weights along a line defined by the direction of the gradient, but placing them at the position on this line where the error function is minimized. This technique is not very efficient since it can be shown that the path taken by $\mathbf{w}$ during the learning process will necessarily zigzag in weight space, taking right angle turns at each step [74].

Several other algorithms also change the weights to the error minimum along a line, but rely on more elaborate methods to determine the direction of this line. One of these algorithms is the conjugate gradient method where the direction is chosen using conjugate directions [74]. Other techniques are based on the quasi-Newton method, which requires the computation of an approximation of the inverse of the Hessian matrix (i.e., the matrix of the second order derivative of the error with respect to the weights) [74]. The computation of this matrix, which has a size equal to the number of weights squared, confer to the quasi-Newton method one of its disadvantages, since it can be very computationally intensive especially for large networks. Furthermore, it should be noted that for some of these two methods, the error is not allowed to increase between two steps. Therefore, if $\mathbf{w}$ reaches a local minimum on the error surface, it is impossible for it to escape and the global minimum cannot be reached. 
For a far more detailed discussion of the different optimization techniques presented above as well as several variations, see C. M. Bishop (1995) [74].

\subsection{ROOT's Multilayer Perceptron Class}

This research project was performed using the multilayer perceptron neural network implementation distributed with the ROOT software [39]. The code was written by C. Delaere and is based on the neural network algorithm from the mlpfit package written by Jerome Schwindling [75]. It is implemented as a C++ class named TMultiLayerPerceptron, which describes a MLP neural network and provides facilities to train it using error back-propagation and multiple optimization algorithms. The error function used is the sum in quadrature of the error on each individual output neuron as presented in Equation 5.8. The neurons of the hidden layers of the MLP can be made with different activation functions, such as sigmoids and tanh, and the output neurons use by default a linear activation function.

For the optimization algorithms used to adjust the weights during the learning process, six options are implemented. One of them, named "stochastic minimization", is the gradient descent method mentioned in the previous section, with the weight adjustment performed on a pattern-by-pattern basis. The default learning rate and momentum constant used by this technique are $\eta=0.1$ and $\mu=0$, respectively. Another one, named "batch", is identical to the stochastic minimization but with the weights incremented at the end of each epoch (batch learning). In the "steepest descent" method, the weights are placed at the point where the error is minimized on the line defined by the direction of the gradient. Two versions of the conjugate gradient method are implemented, named "Polak-Ribiere" and "Fletcher-Reeves". Finally, an algorithm based on the quasi-Newton method named "Broyden, Fletcher, Goldfarb, Shanno" (BFGS) is also available. All these algorithms, except for the 
stochastic minimization, are used with the batch learning method.

\subsection{The Neural Network Analysis}

An application was developed to use the ROOT TMultiLayerPerceptron class presented in the previous section to study the potential of multilayer perceptron neural networks to help with event reconstruction in the XEP experiment. The application uses tracks generated by the simulation presented in Chapter 3 for training and testing the network.

The simulation data consists of the pad number and arrival time of the EL photons on the photocathode closest to the EL region. The position along the z-axis are obtained from the product of the arrival time and the drift velocity. This way, a three dimensional representation of the track is obtained, similar to the examples shown in Section 3.5 .

To be used as the neural network input, the data must first be formatted in a way where each entry represents an event. The volume in the detector where the tracks are located is divided into a certain number of bins, each of which will be associated with an input neuron. The dimensions of the pads on the photocathode already bins the data in the $x-y$ plane. For the $z$-axis, the minimum and maximum positions of the tracks along this axis are calculated and the distance in-between is separated into a number of bins, usually 10 or 12 . This produces a different bin size for all tracks, but ensures that, along this axis, the tracks occupies every layer of bins. Given the typical size of tracks, the bin size is usually less than a centimetre. This is much larger than the binning that should be produced by the digitization of the signal in the actual XEP detector.

Now, the information about each event is represented by a specific number of bins containing a number of photon hits. Each of these bins is associated with one of the 
input neurons of the neural network, forming each element of the input vector. In addition, one or more other values are added to each entry of the TTree object to define the output targets used during the training.

The photocathode contains 232 pads. Multiplying this value by the number of bins along the z-axis gives the number of input neurons of the network. For example, if the $\mathrm{z}$-axis is divided into 12 bins, the input layer will have 2784 neurons. Moreover, if the network has $N_{1}$ neurons in its first hidden layer, their will be $2784 \times N_{1}$ synaptic weights between the first two layers of neurons. These large numbers make the training of the neural network very computationally intensive, both in term of CPU time and memory usage. It is this factor that limits choice of the number of z-axis bins and motivated the choice of variable bin size so that as much information about the tracks as possible could be included in the input vector.

Before starting to train the neural network, the entries of the data set must be separated into a training set and a test set. The first set is used during the training process to evaluate the errors and adjust the weights. The second set is used to test the validity of the training. The performance of a neural network has to be generalized, meaning that it needs to be able to approximate the desired output for input vectors that are not part of the training set.

\subsection{Number of Track Classification}

As mentioned in chapter 2, part of the background discrimination will consist in identifying whether a given event is formed of two tracks, such as neutrinoless or twoneutrino double beta decay, or of a single track, in which case the event is necessarily background. This section presents a study of the performance of the neural network in distinguishing between these two categories of events.

The data used consist of neutrinoless double beta decay events and single electron 
events generated with an energy equal to the $\beta \beta(0 \nu)$ Q-value of $2457.8 \mathrm{keV}$. Both types of event were simulated in xenon at 10 atmospheres with a drift electric field of $1 \mathrm{kV} \mathrm{cm}{ }^{-1}$ and an EL electric field of $40 \mathrm{kV} \mathrm{cm}^{-1}$. However, to be able to generate the data in a reasonable amount of time, the ionization was reduced by a factor of 100 and the electroluminescence yield by a factor of 51.3 , producing an average of about 96,000 EL photons per event. An energy cut was applied to reject tracks that were not entirely contained in the fiducial volume, which left 17688 single electron and $22193 \beta \beta(0 \nu)$ events. Both categories of events were separated in half between the training and the validation data sets, and the z-axis was divided in 12 bins. Finally, the networks possessed a single output neuron and the target outputs provided for the training were encoded as follows: 0 represented a one electron event while 1 represented a $\beta \beta(0 \nu)$ event.

All the learning algorithms available were previously tested and the stochastic minimization and the BFGS methods presented the fastest learning rates. Several network structures were tested as well. Either a single hidden layer of 2, 5, or 10 neurons, or two layers both of $2,5,10$, or 50 neurons were used. Within this range, the number of neurons as well as the number of layers did not have a clear impact on the learning rate of the network.

The following figures present the results obtained for a sample of the network structures and learning methods used. In each of them, the histograms show the output distribution for the testing data set for each of the two categories of events. Every time the network is presented with one of the event of this set, it calculates an output, which, if the training was successful, should be close to 0 for one electron events and close to 1 for $\beta \beta(0 \nu)$ events.

If the neural network was used with data from the real detector, the actual category of each event would not be known and a choice would have to be made to decide, based on the network outputs, which ones should be rejected and which ones should 
be kept. For this, a cut could be placed at a certain value between 0 and 1 , and all events for which the network output is below the cut would be assumed to be from single electrons, while all events with an output above the cut would be assumed to be $\beta \beta(0 \nu)$. The two lines in the figures show, as a function of the applied cut, the ratio of single electron events calculated above the cut, which would be mistaken for $\beta \beta(0 \nu)$, and the ratio of $\beta \beta(0 \nu)$ events below the cut, which would be mistaken for background.

To begin, Figures 5.3 to 5.5 present the results for neural networks with two layers of hidden neurons, each of 5,10 , or 50 neurons. The training was performed with the stochastic minimization method and 50 epochs.

As mentioned above, the network structure had no clear impact on the learning rate. However, as can be seen, it affects the shape of the output distribution. Networks with more neurons produce smoother distributions than networks with fewer

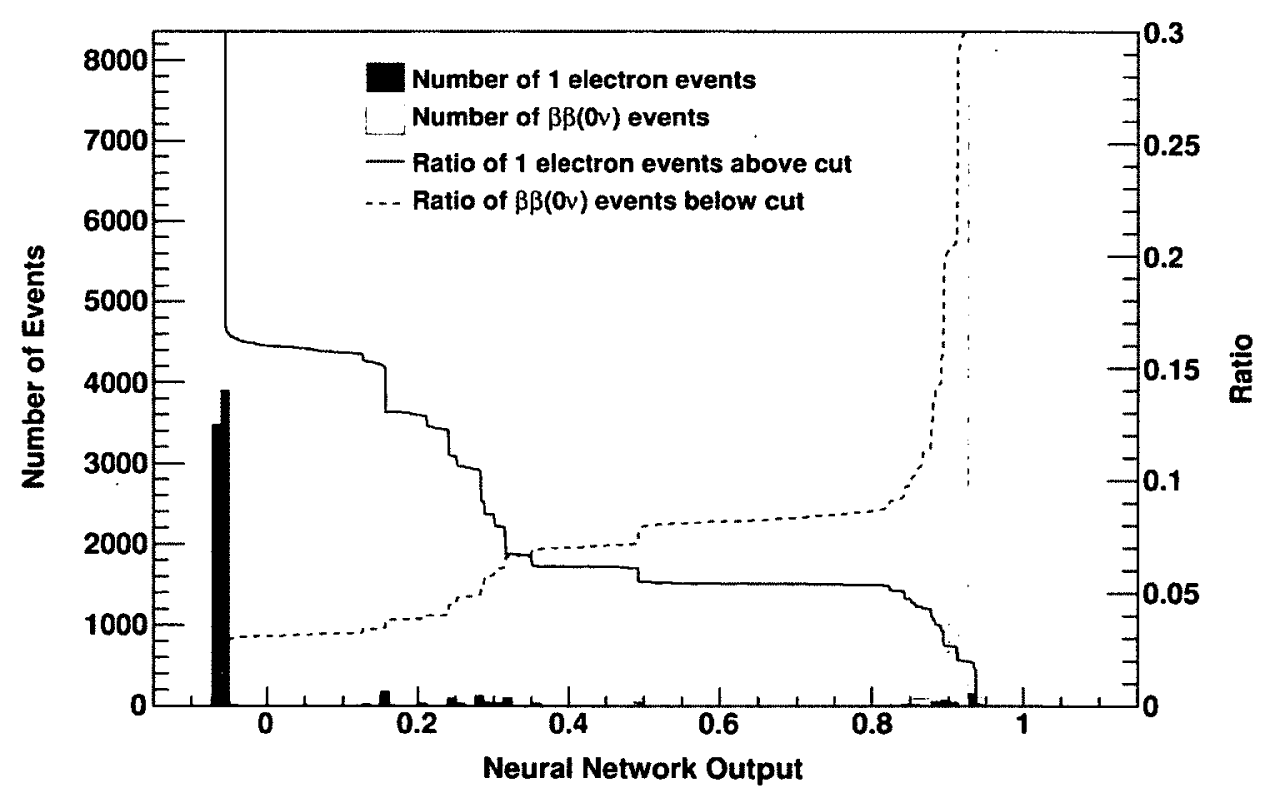

Figure 5.3: Distribution of output from a neural network with two hidden layers of 5 neurons each trained with the stochastic minimization algorithm over 50 epochs. 


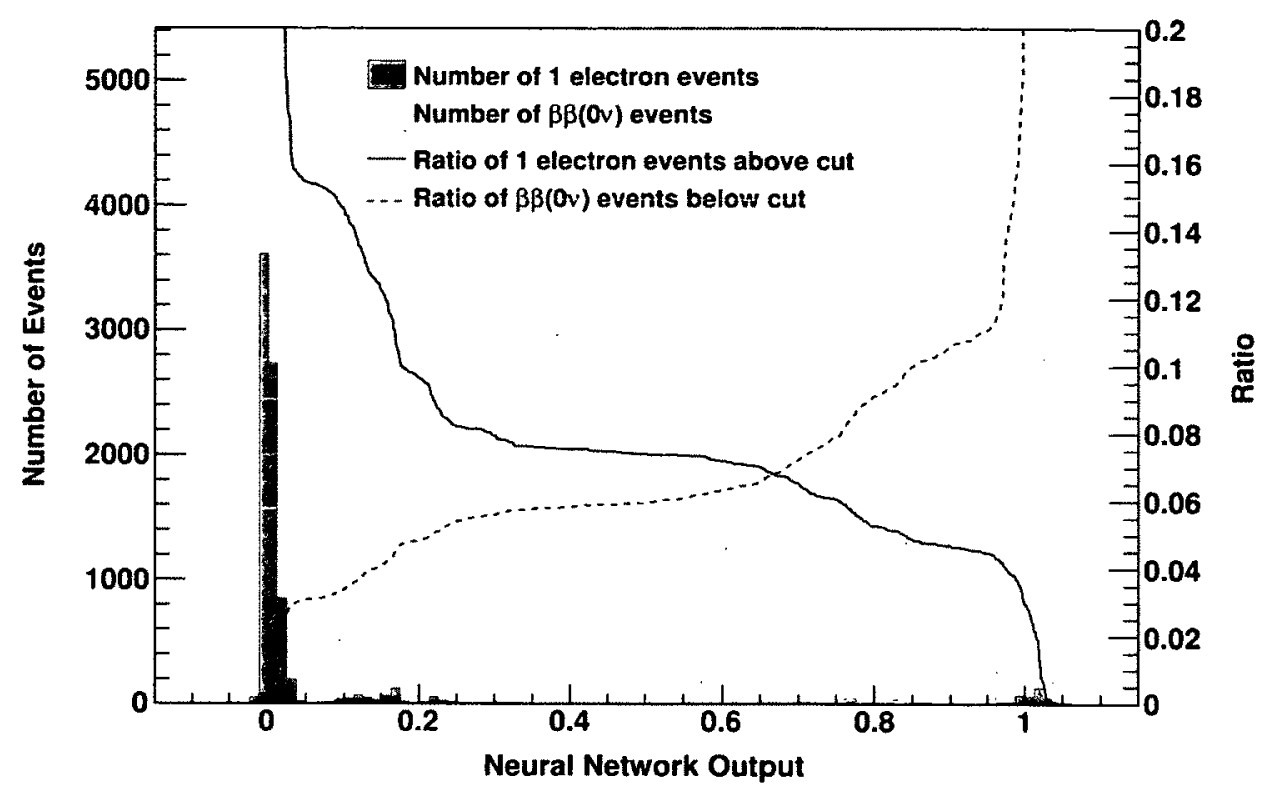

Figure 5.4: Distribution of output from a neural network with two hidden layers of 10 neurons each trained with the stochastic minimization algorithm over 50 epochs.

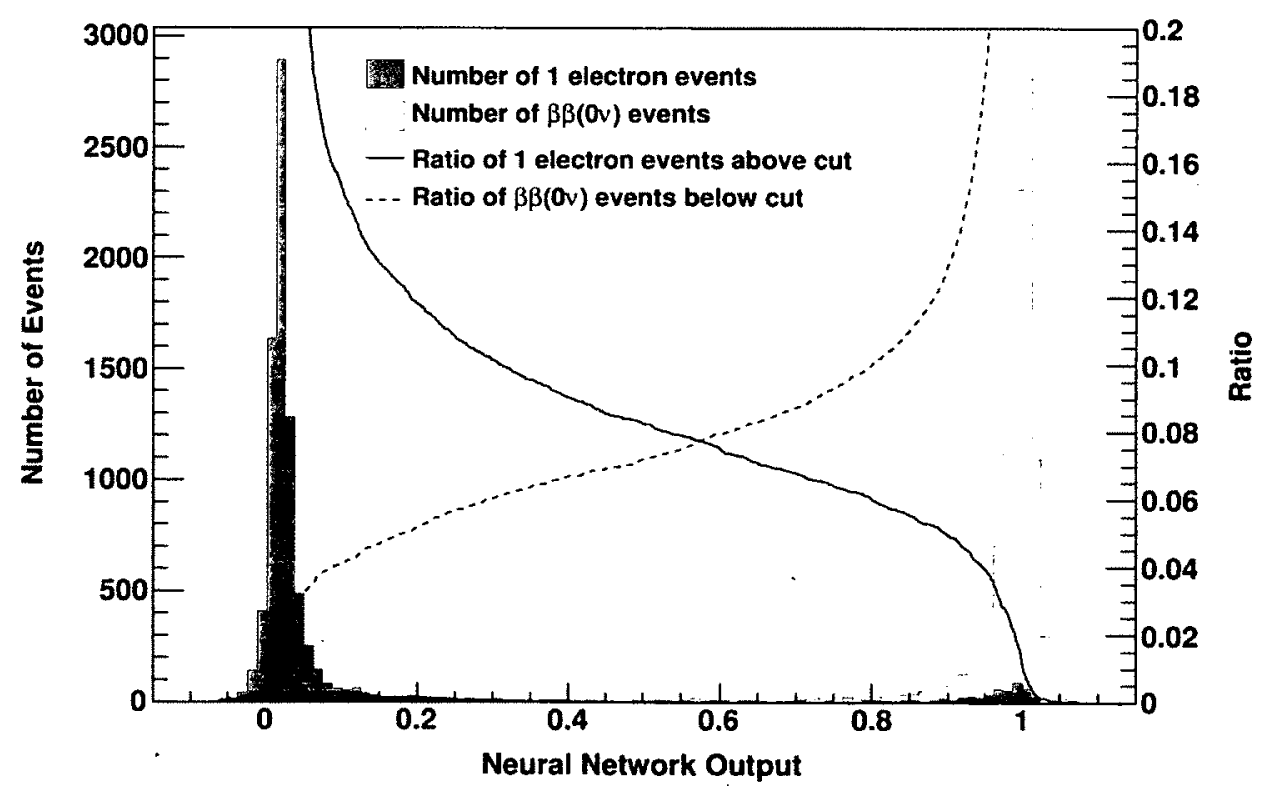

Figure 5.5: Distribution of output from a neural network with two hidden layers of 50 neurons each trained with the stochastic minimization algorithm over 50 epochs. 
neurons. This becomes even more apparent in the simplest network tested, which had a single hidden layer of 2 neurons. The result is shown in Figure 5.6. This partial discretization of the output values is believed to be associated with the asymptotic nature of the activation functions. If the weighted summation of Equation 5.2 produces values over a wide range, a neuron would produce outputs most often near the asymptotes of it's activation function ( 0 or 1 for the sigmoid), acting approximately like a step function. If this happens at the neurons of the last hidden layer, the output of the neural network, which is a linear combination of the outputs of the neurons of the last hidden layer, would produces numbers most often near $2^{N}$ different values, where $N$ is the number of neurons in the last hidden layer. This hypothesis seems to agree well at least with Figures 5.3 and 5.6.

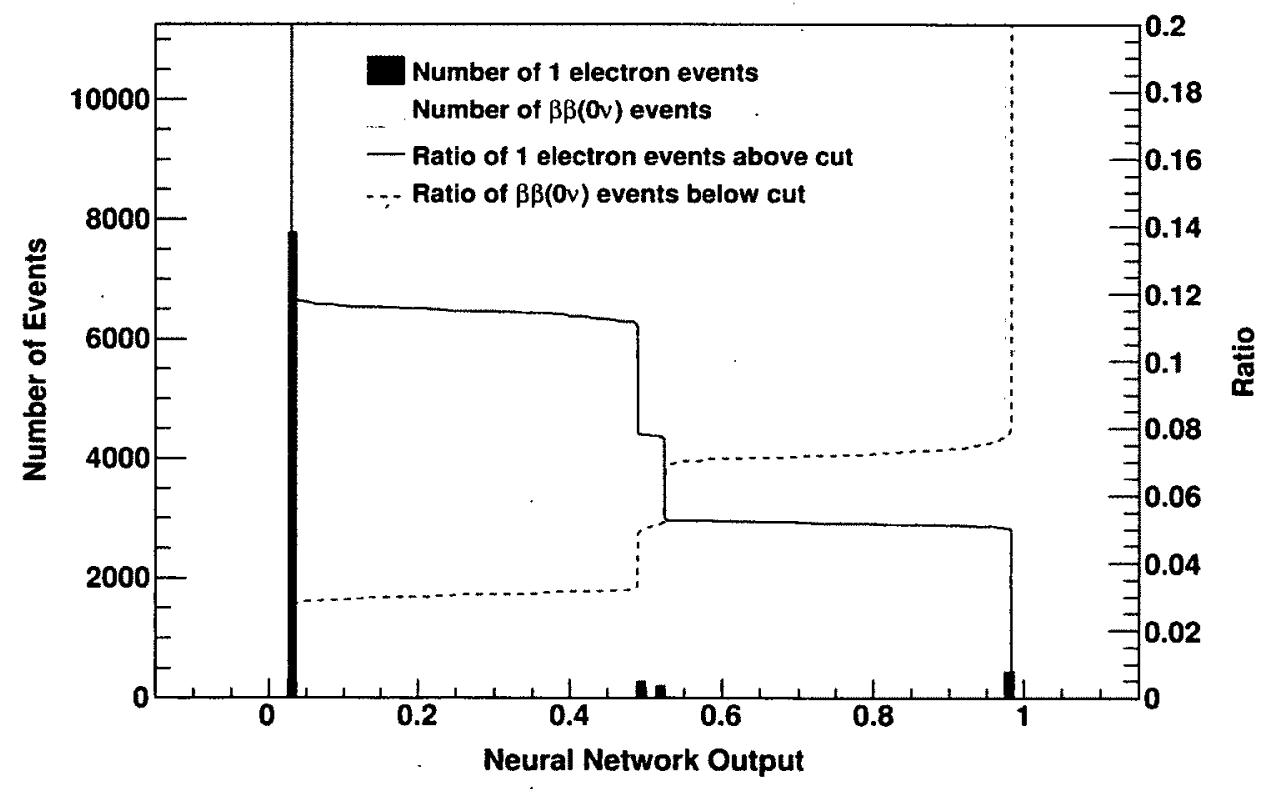

Figure 5.6: Distribution of output from a neural network with a single layer of 2 neurons trained with the stochastic minimization algorithm over 50 epochs.

Although the Stochastic minimization and the BFGS method produced similar results in term of training rate, BFGS presents a serious limitation in the complexity of 
the networks that can be trained with it. This is believed to be due to the requirement by this minimization algorithm to create and store the hessian matrix, which, for a network with $W$ weights, has $W^{2}$ elements. In the case of the networks presented in this section with 10 hidden neurons, a matrix of $(2784 \times 10+10 \times 1)^{2} \approx 776$ million elements needed to be defined. The most complex neural network that could be successfully trained with this algorithm had two layers of five neurons each. The results are presented in Figure 5.7.

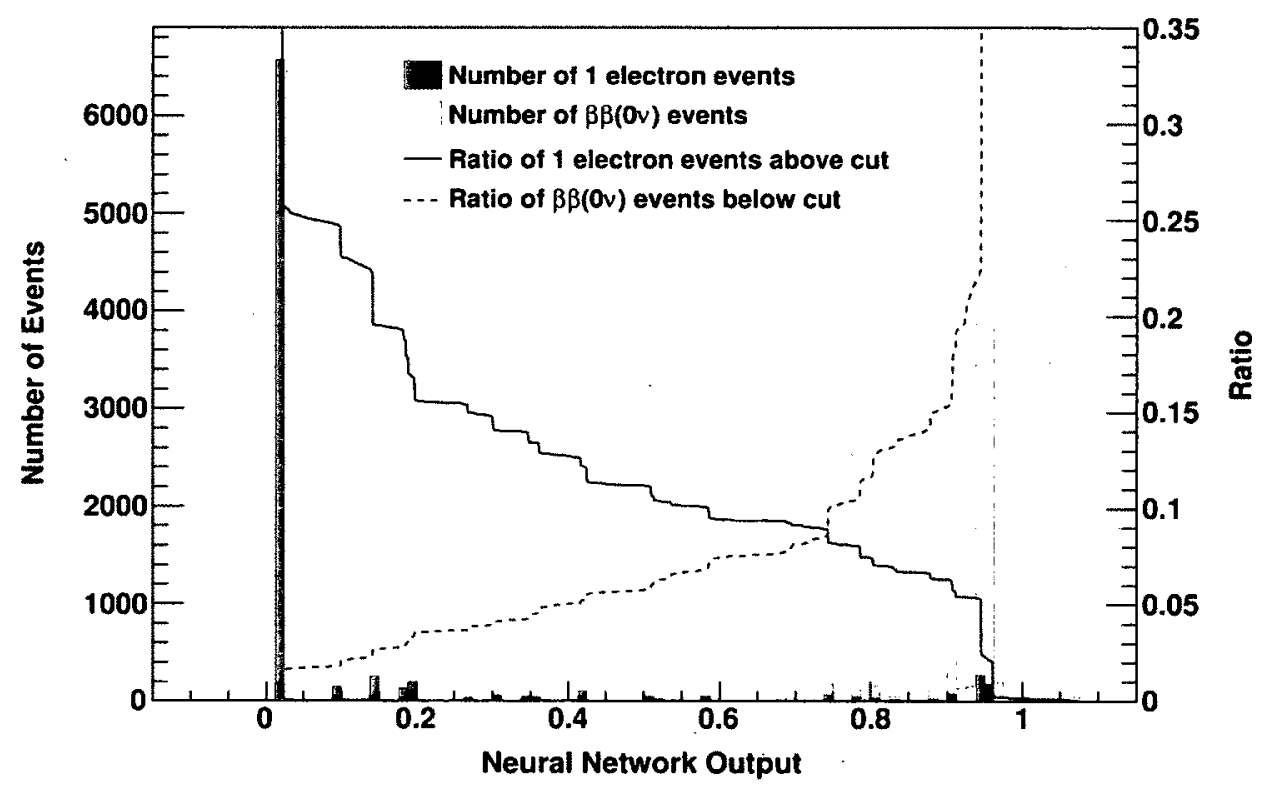

Figure 5.7: Distribution of output from a neural network with two layers of 5 neurons each with the BFGS method over 100 epochs.

From the results obtained, it appears that the neural networks presented above are adequate to separate $\beta \beta(0 \nu)$ events from single electron tracks of $2457.8 \mathrm{keV}$ at $10 \mathrm{~atm}$ with a certain accuracy. For example, in the results obtained with two layers of 10 neurons and the stochastic minimization training (Figure 5.4), if a cut of 0.5 was chosen, about $7.4 \%$ of the 1 electron events would be mistaken for $\beta \beta(0 \nu)$ and about $6.0 \%$ of the $\beta \beta(0 \nu)$ would be mistaken for single electron events. The best cut 
value to choose will depend on the actual background rate that will be observed in the region of interest.

\subsection{Multi-Site Background Rejection}

If two background tracks form in the detector sufficiently close to each other or within a sufficiently small time interval, the resulting signal could be mistaken for double beta decay. This section presents a study of the performance of the neural network for distinguishing between $\beta \beta(0 \nu)$ events and events composed of two electrons of distinct but close origins.

The neural network was trained with $6000 \beta \beta(0 \nu)$ and 6000 multi-site events simulated at 10 atmospheres. The multi-site events consisted of two electrons generated within a distance of 0.5 to $2 \mathrm{~cm}$ and a time interval of 0 to $5 \mu$ s from each other and with a total energy equal to the $\beta \beta(0 \nu) \mathrm{Q}$ value. As in the previous section, the ionization and EL yield were scaled down so that approximately 96,000 EL photons per events were produced. The neural networks used had a first hidden layer of either 2, 4, or 6 neurons. Half of them had only one hidden layer while the other half had a second layer of 4 neurons. The output layer consisted of a single neuron with the target values encoded in a binary way: 0 for the background events and 1 for the $\beta \beta(0 \nu)$. Again, within the range investigated, the number of neurons did not have a clear impact on the training speed, other than the discrete structure visible in the output distribution of the simplest networks as discussed in the previous section. Furthermore, the stochastic minimization and the BFGS methods offered again the best learning rates.

Similarly to the plots of the previous section, the following figures show, for some of the network structures and learning methods used, the histogram of the output distribution together with the plots of the ratio of events mistaken for the wrong 


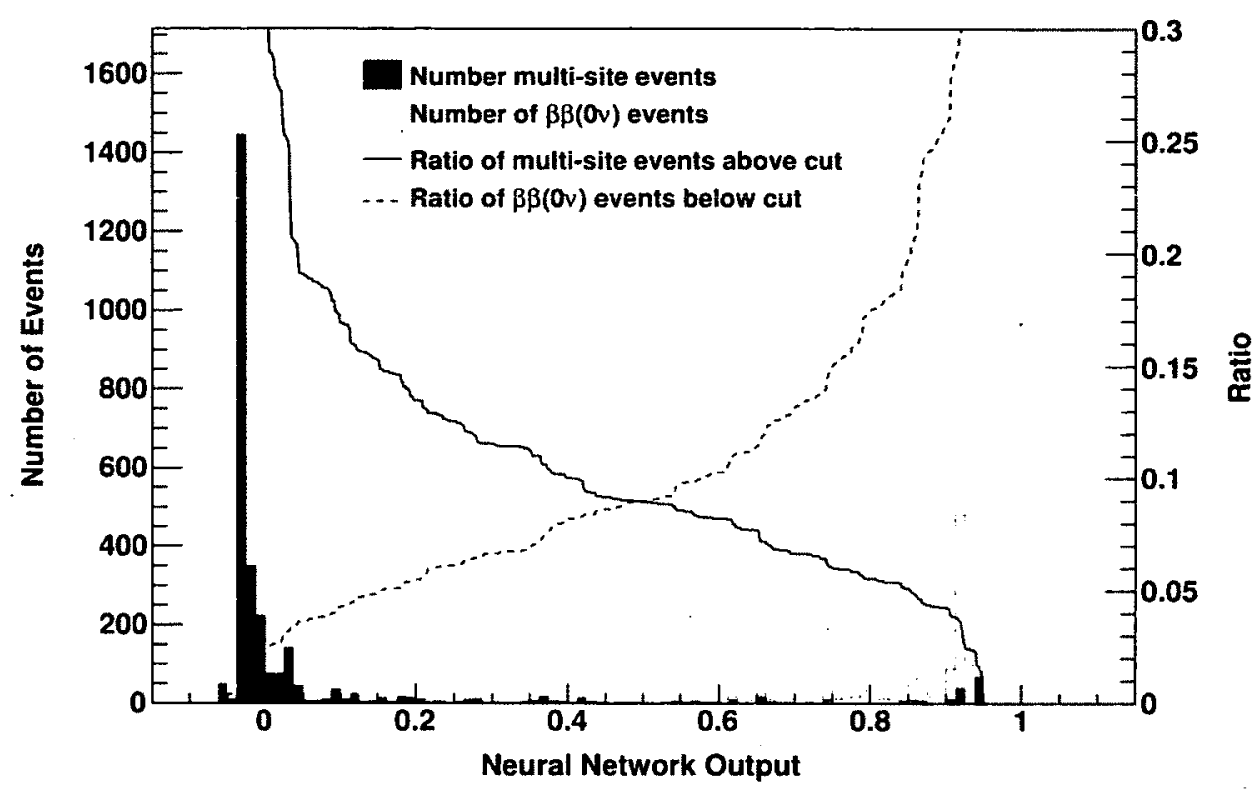

Figure 5.8: Distribution of the output of a neural network with two hidden layers of 6 and 4 neurons trained with the stochastic minimization and 50 epochs.

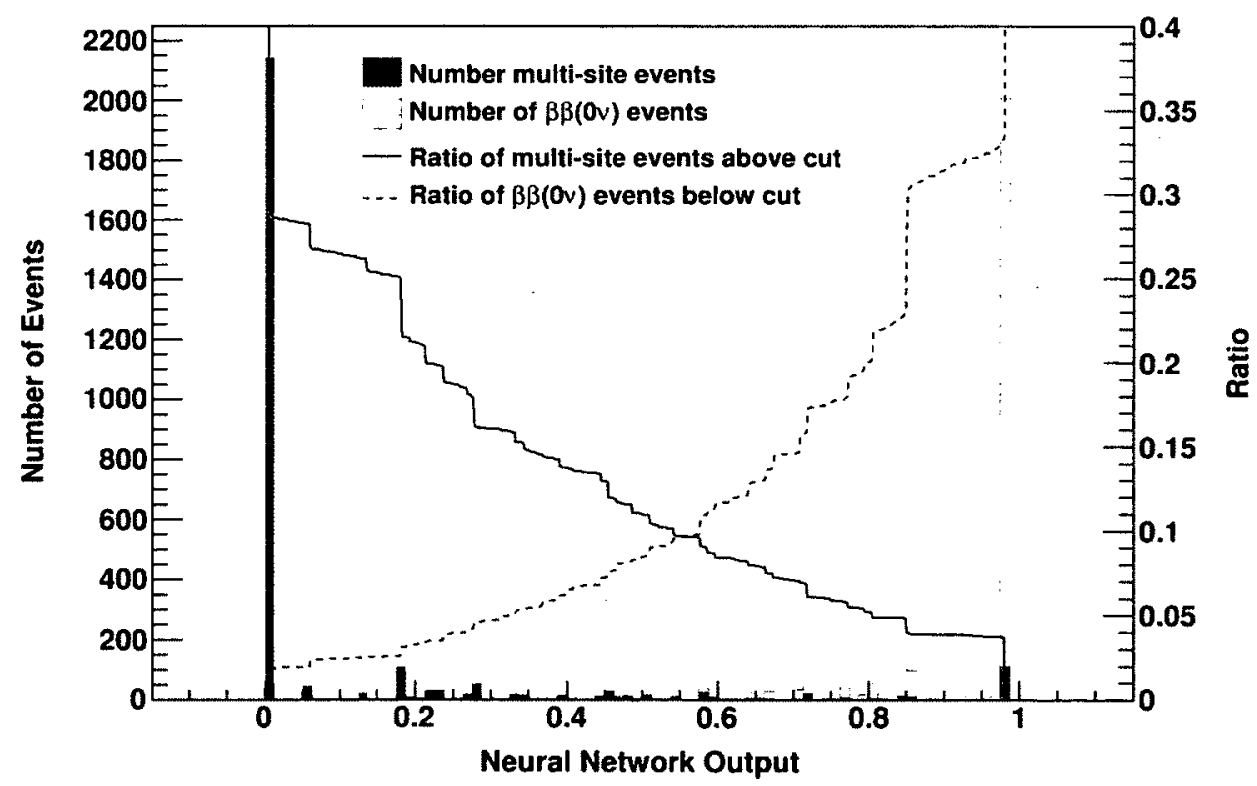

Figure 5.9: Distribution of the output of a neural network with two hidden layers of 6 and 4 neurons trained with the BFGS method and 100 epochs. 
category as a function of the selection cut applied. Both figures present the results of a network made of two hidden layers of 6 and 4 neurons respectively. In Figure 5.8, the network was trained with the stochastic minimization algorithm over 50 epochs, while in Figure 5.9 it was trained using the BFGS method over 100 epochs.

As can be seen, it was possible for the neural network to discriminate between events formed of two tracks of joint and disjoint origin simulated at $10 \mathrm{~atm}$. As in the previous section, the efficiency of the algorithm would depend on the choice of cut applied on the network output values. For example, in the case of the network trained with the stochastic minimization method (Figure 5.8), a cut placed at 0.5 would result in about $9 \%$ of the both the multi-site background events and the $\beta \beta(0 \nu)$ events to be mistaken for the wrong category. Again, the best cut value to choose will depend on the rate of background events that will contaminate the results.

\subsection{Vertex Reconstruction}

This section presents a study of the performance of the neural network for finding the point of origin of electron tracks in the detector. The neural network was used to identify the vertex location of $1 \mathrm{MeV}$ electrons simulated in xenon at $5 \mathrm{~atm}$. To simplify the problem, the tracks were produced only within a spherical volume $15 \mathrm{~cm}$ of diameter at the center of the TPC.

It was found that to achieve any success with vertex reconstruction, the neural network had to be significantly more complex than in the previous sections. Due to computing limitations, the largest networks that could be trained had 2320 neurons in their input layer and 60 neurons in their first hidden layer. The only exception was for networks trained with the BFGS method, which could not attempt to train with more than between 10 and 20 neurons in their first hidden layer. This is likely due to the size of the Hessian matrix computed by this algorithm as mentioned in 
Section 5.1.3. The networks used in this analysis had two output neurons for the $\mathrm{x}$ and $y$ coordinates of the vertex. It is often suggested that scaling the target values within the range of the activation function can accelerate the training process. Therefore, both vertex coordinates were scaled down by a factor of 0.12 to make all values be within the interval $[-0.9,0.9]$.

Stochastic minimization showed again the best learning rate and it is only with this algorithm that any success was achieved. The batch method presented the slowest learning rate, but was still slowly converging after 20 epochs of training. The error for networks trained with the two conjugate gradient algorithms appeared to reach a plateau after only four or five epochs, after which the error remained almost constant for the rest of the training. It is possible that these minimization algorithms reached a local minimum of the error surface, from where they could not escape, as discussed in Section 5.1.3.

The following figures show the results for two of the neural networks that provided the best outcomes after having being trained over 20 epochs. One had a single layer of 50 neurons, while the other had two layers of 60 neurons each. Figures 5.10 and 5.11 show the scatter plots of the output of the neural network as a function of the target values. The correlation coefficient was calculated in each cases, defined as

$$
\rho\left(x_{\text {true }}, x_{\text {calc }}\right)=\frac{\operatorname{cov}\left(x_{\text {true }}, x_{\text {calc }}\right)}{\sigma\left(x_{\text {true }}\right) \sigma\left(x_{\text {calc }}\right)}
$$

where $x_{\text {true }}$ and $x_{\text {calc }}$ are the true (target) and calculated (neural network's output) values respectively, and $\operatorname{cov}\left(x_{\text {true }}, x_{\text {calc }}\right)$ is the covariance. In the first case (Figure 5.10), $\rho\left(x_{\text {true }}, x_{\text {calc }}\right)=0.74$ and $\rho\left(y_{\text {true }}, y_{\text {calc }}\right)=0.75$, while in the second case (Figure 5.11) $\rho\left(x_{\text {true }}, x_{\text {calc }}\right)=0.77$ and $\rho\left(y_{\text {true }}, y_{\text {calc }}\right)=0.78$.

Figures 5.12 and 5.13 show the distribution of the distance between the true and 

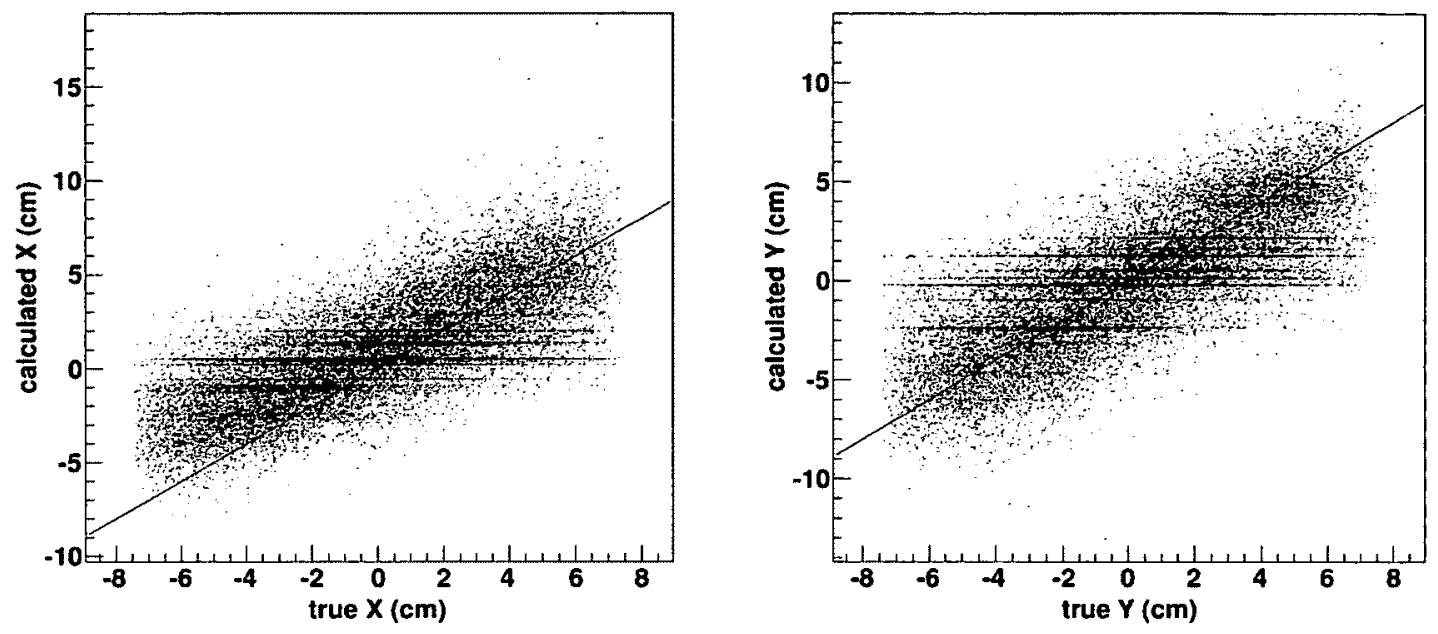

Figure 5.10: Scatter plot of the calculated output as a function of the target value (true value) for a neural network with a single layer of 50 neurons trained over 20 epochs with the stochastic minimization algorithm.
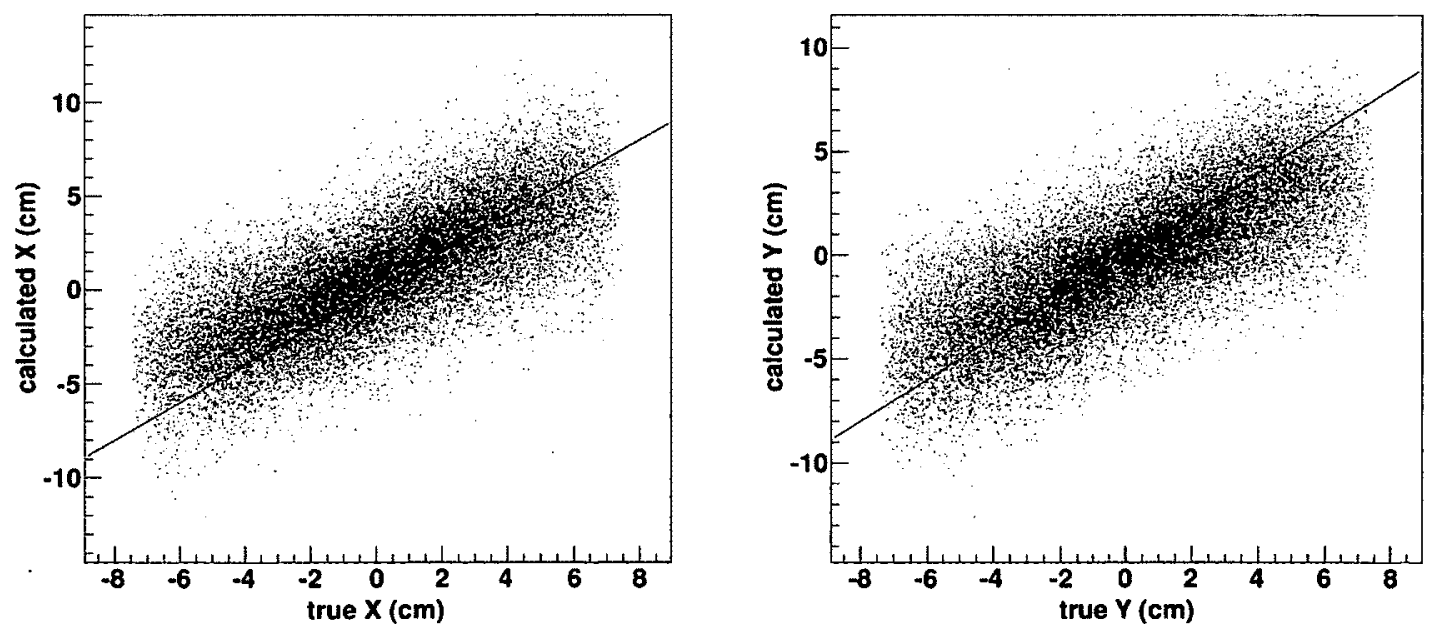

Figure 5.11: Scatter plot of the output calculated as a function of the target value for a neural network with two hidden layers of 60 neurons each trained with the stochastic minimization method over 20 epochs. 
the calculated positions for the two network structures mentioned above. The distributions of the distances along the $\mathrm{x}$ and the $\mathrm{y}$ axes were fitted with a Gaussian function to find the width of the distribution. The fit parameters are listed in Tables 5.1 and 5.2. Note that the widths calculated this way seem slightly overestimated and their uncertainties underestimated because the distributions are not perfectly Gaussian.

The widths of the distributions in Tables 5.1 and 5.2 show that the neural network can be used to reduce significantly the range where the point of origin of an electron track can be expected. $1 \mathrm{MeV}$ electrons were generated in 5 atm xenon within a spherical volume of $15 \mathrm{~cm}$ of diameter, and the difference between the actual position of their vertex and the ones reconstructed by the network had, in the best case, a width of $2.15 \pm 0.01 \mathrm{~cm}$ and $2.10 \pm 0.01 \mathrm{~cm}$ along the $\mathrm{x}$ and $\mathrm{y}$ axis, respectively. The results also display biases of $0.637 \pm 0.013 \mathrm{~cm}$ and $-0.305 \pm 0.012 \mathrm{~cm}$ on the $\mathrm{x}$ and $\mathrm{y}$ axis, respectively, expressed by the mean values in Tables 5.2. Further investigation is required to understand the cause and significance of these biases.

Trying to locate the origin of single electron tracks was only a first step toward realizing the vertex reconstruction of $\beta \beta(0 \nu)$ events. This task will likely be more challenging, but would allow to locate the barium ion daughter of the decay. This information would be very useful for the barium tagging technique currently being researched in order to observe the ion in the volume of the TPC. 


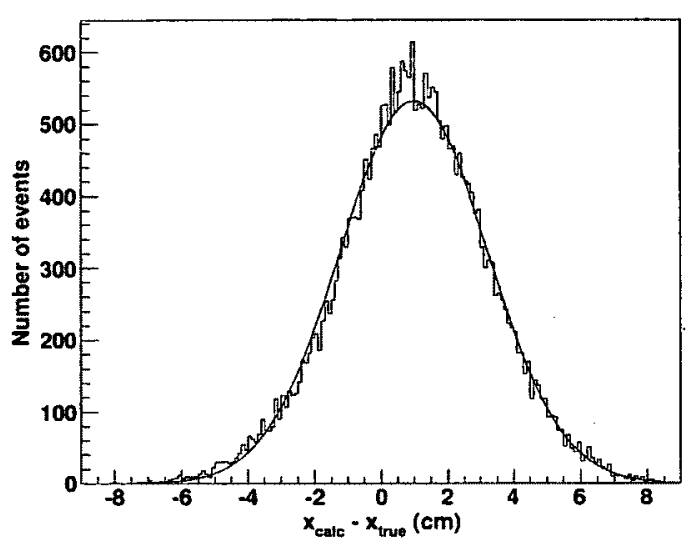

(a)

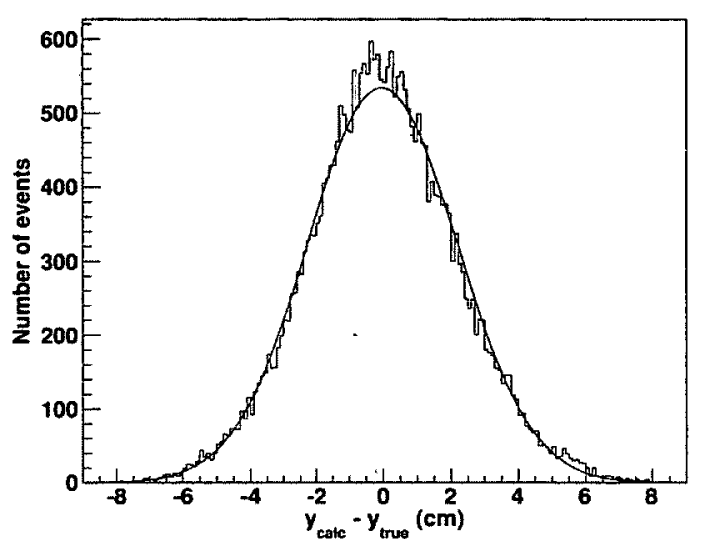

(b)

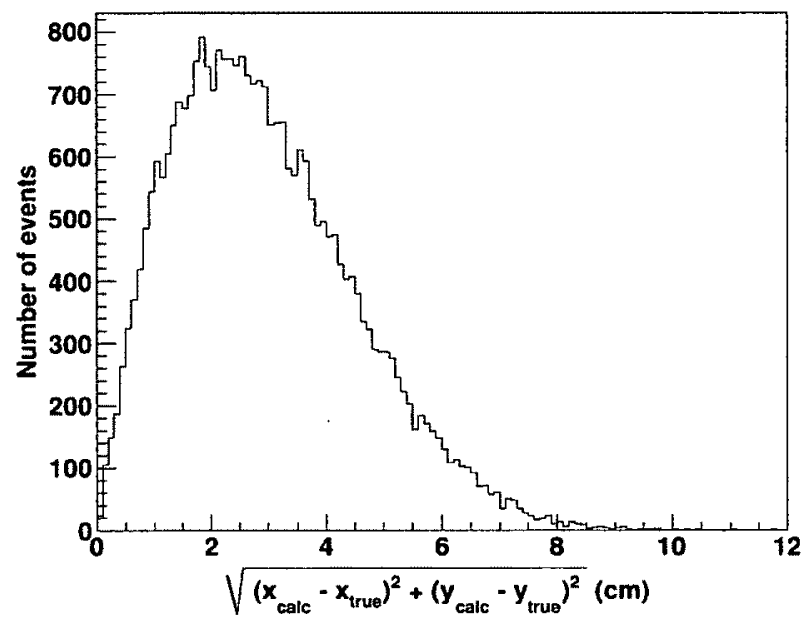

(c)

Figure 5.12: Distribution of the difference between the output and the target values of a neural network with a single layer of 50 neurons trained with the stochastic minimization algorithm over 20 epochs.

\begin{tabular}{ccc} 
& Mean & Standard Deviation \\
\hline $\mathrm{x}_{\text {calc }}-\mathrm{x}_{\text {true }}$ & $0.990 \pm 0.013 \mathrm{~cm}$ & $2.22 \pm 0.01 \mathrm{~cm}$ \\
$\mathrm{y}_{\text {calc }}-\mathrm{y}_{\text {true }}$ & $-0.044 \pm 0.013 \mathrm{~cm}$ & $2.22 \pm 0.01 \mathrm{~cm}$
\end{tabular}

Table 5.1: Fit parameters of the fits from Figures 5.12a and 5.12b. 


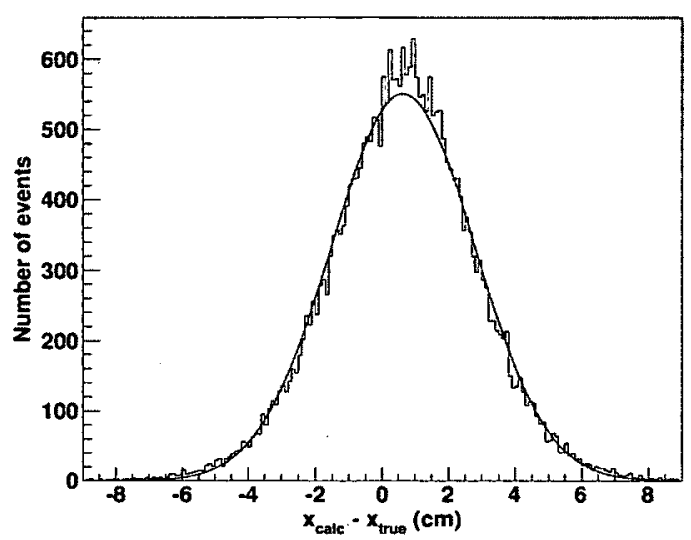

(a)

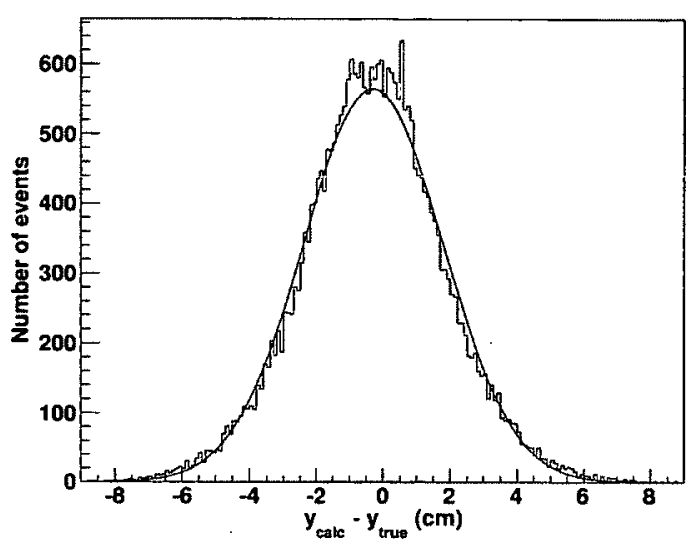

(b)

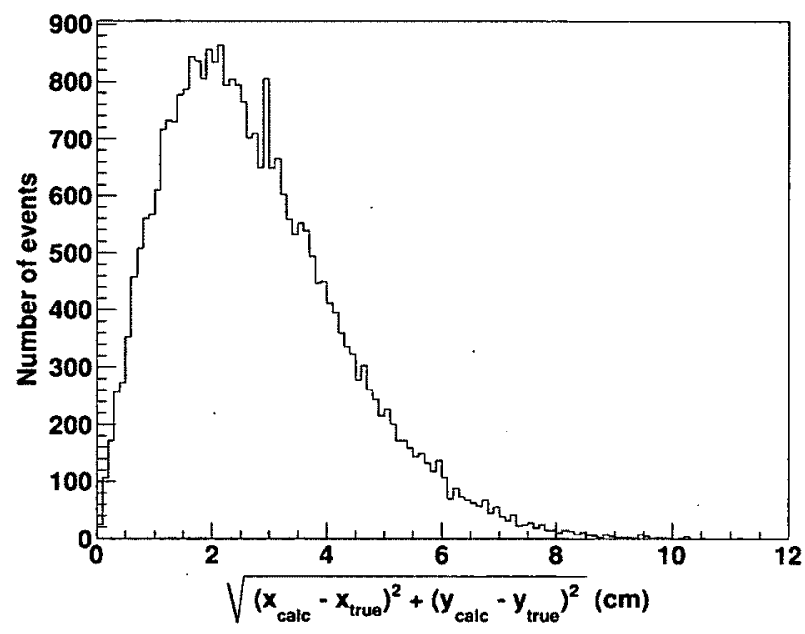

(c)

Figure 5.13: Distribution of the difference between the output and the target values of a neural network with two layers of 60 neurons trained with the stochastic minimization algorithm over 20 epochs.

\begin{tabular}{ccc} 
& Mean & Standard Deviation \\
\hline $\mathrm{x}_{\text {calc }}-\mathrm{x}_{\text {true }}$ & $0.637 \pm 0.013 \mathrm{~cm}$ & $2.15 \pm 0.01 \mathrm{~cm}$ \\
$\mathrm{y}_{\text {calc }}-\mathrm{y}_{\text {true }}$ & $-0.305 \pm 0.012 \mathrm{~cm}$ & $2.10 \pm 0.01 \mathrm{~cm}$
\end{tabular}

Table 5.2: Fit parameters of the fits from Figures 5.13a and 5.13b. 


\section{Chapter 6}

\section{Conclusion}

This thesis presented the work done to build a general Monte Carlo simulation of the gaseous xenon TPC prototype developed as part of the EXO experiment. After performing some validation tests, the simulation was utilized to perform multiple studies.

To begin, the simulation was used to study certain aspects of the uniformity of EL photon detection on both photocathodes for light originating at different positions along the EL region plane. When simulating single ionization electrons, it was found that variations as high as $20 \%$ occurred in the detected light signal on the anode side photocathode. These fluctuations were dominated by the position of the drifting electrons with respect to the Teflon spacer grid of the readout package. However, for tracks detected in the TPC, these variations in response will be averaged over a large number of ionization electrons. On the photocathode at the other end of the detector, the efficiency was found to be $8.4 \%$ lower for photons generated near the edge of the EL region than for photons emitted at the center.

The simulation was also used to estimate the background rate in the TPC from the radioisotopes in the concrete surrounding the detector and from cosmic muons directly hitting the detector. With the current gamma shielding in place, background rates of 1630 and 5020 counts/s were estimated depending on the choice of activity 
concentration assumed to be present in the concrete. Since this background could result in a high pile-up rate, particularly at high pressure, it might be preferable to increase the shielding around the detector.

To calibrate the TPC, calibration gamma sources with high strengths would allow to decrease the time required to perform the calibration. However, a high detection rate can result in a high pile-up fraction in the readout. Therefore, the simulation was used to investigate what source strength would be suitable to use.

Furthermore, a multilayer perceptron neural network algorithm was used to analyze data from the Monte Carlo simulation. The neural network was able to distinguish $\beta \beta(0 \nu)$ from both one and two electron background events of energy equal to the decay $Q$ value. In light of the results described in this thesis, neural networks seem like a promising tool for discriminating background events and determining the likelihood of a track to be $\beta \beta(0 \nu)$ or $\beta \beta(2 \nu)$.

The neural network was also used to determine the starting point of single electron tracks. For $1 \mathrm{MeV}$ electrons simulated at $5 \mathrm{~atm}$, in the best case the difference between the actual positions of the vertex and the ones calculated by the neural network formed a distribution with a width of $\sigma_{x}=2.15 \mathrm{~cm}$ along the x-axis and $\sigma_{y}=2.10 \mathrm{~cm}$ along the $y$-axis.

Several factors should be added to the simulation in order to reproduce the full signal generation process. This includes electron losses in the gas due to electronegative impurities, the charge gain process in methane, and the effects of the electronics, including the noise. Also, some components of the detector, which could affect the light propagation are missing from the simulation, such as the wire meshes used for producing the electric fields in the EL region and in the methane gap. The addition of those effects will be the subject of further work.

The initial investigation of utilizing the neural network has produced interesting results. However, more work will be done to evaluate its capacity in distinguishing 
signal and background. For example, the study of background rejection should be carried out on a broader range of experimental conditions, such as the gas pressure, to ensure that the neural network can also perform in those conditions. Moreover, the discrimination of more types of background with similar signal to double beta decay events should be attempted.

Finally, the study of vertex reconstruction of single electron tracks was a first step toward the goal of being able to locate the vertex of $\beta \beta(0 \nu)$ events. This task would be useful for the barium tagging technique which, by observing the barium ion daughter of the decay would discriminate all sources of background. 


\section{References}

[1] K. M. Heeger, "Evidence for Neutrino Mass: a Decade of Discovery," in Proceeding of the International Conference on the Seesaw Mechanism, Institut Henri Poincar, Paris, 2004, J. Orloff, S. Lavignac, and M. Cribier, Eds., 2005, pp. 65-80.

[2] C. L. Cowan, Jr. et al., "Detection of the Free Neutrino: A Confirmation," Science, vol. 124, pp. 103-104, 1956.

[3] G. Danby et al., "Observation of High-Energy Neutrino Reactions and the Existence of Two Kinds of Neutrinos," Phys. Rev. Lett., vol. 9, pp. 36-44, 1962.

[4] M. L. Perl et al., "Evidence for anomalous lepton production in $e^{+}-e^{-}$annihilation," Phys. Rev. Lett., vol. 35, pp. 1489-1492, 1975.

[5] K. Kodama et al., "Observation of tau neutrino interactions," Physics Letters B, vol. 504, pp. $218-224,2001$.

[6] H. A. Bethe and C. L. Critchfield, "The formation of deuterons by proton combination," Phys. Rev., vol. 54, pp. 248-254, 1938.

[7] R. Davis, D. S. Harmer, and K. C. Hoffman, "Search for neutrinos from the sun," Phys. Rev. Lett., vol. 20, pp. 1205-1209, 1968.

[8] K. S. Hirata et al., "Real-time, directional measurement of ${ }^{8} \mathrm{~B}$ solar neutrinos in the kamiokande ii detector," Phys. Rev. D, vol. 44, pp. 2241-2260, 1991.

[9] P. Anselmann et al., "Solar neutrinos observed by gallex at gran sasso," Physics Letters B, vol. 285, pp. 376 - 389, 1992.

[10] J. N. Abdurashitov et al., "Measurement of the solar neutrino capture rate with gallium metal," Phys. Rev. C, vol. 60, p. 055801, 1999.

[11] V. Gribov and B. Pontecorvo, "Neutrino astronomy and lepton charge," Physics Letters B, vol. 28, pp. 493 - 496, 1969. 
[12] Z. Maki, M. Nakagawa, and S. Sakata, "Remarks on the unified model of elementary particles," Progress of Theoretical Physics, vol. 28, pp. 870-880, 1962.

[13] Y. Fukuda et al., "Evidence for oscillation of atmospheric neutrinos," Phys. Rev. Lett., vol. 81, pp. 1562-1567, 1998.

[14] Q. R. Ahmad et al., "Measurement of the rate of $\nu_{e}+d \rightarrow p+p+e^{-}$interactions produced by ${ }^{8} \mathrm{~B}$ solar neutrinos at the sudbury neutrino observatory," Phys. Rev. Lett., vol. 87, p. 071301, 2001.

[15] K. Eguchi et al., "First results from kamland: Evidence for reactor antineutrino disappearance," Phys. Rev. Lett., vol. 90, p. 021802, 2003.

[16] M. Apollonio et al., "Search for neutrino oscillations on a long base-line at the chooz nuclear power station," The European Physical Journal C-Particles and Fields, vol. 27, pp. 331-374, 2003.

[17] F. Boehm et al., "Final results from the palo verde neutrino oscillation experiment," Phys. Rev. D, vol. 64, p. 112001, 2001.

[18] K. Abe et al., "Indication of electron neutrino appearance from an acceleratorproduced off-axis muon neutrino beam," Phys. Rev. Lett., vol. 107, p. 041801, 2011.

[19] B. Aharmim et al., "Electron energy spectra, fluxes, and day-night asymmetries of ${ }^{8} \mathrm{~b}$ solar neutrinos from measurements with nacl dissolved in the heavy-water detector at the sudbury neutrino observatory," Phys. Rev. C, vol. 72, p. 055502, 2005 .

[20] Y. Ashie et al., "Measurement of atmospheric neutrino oscillation parameters by super-kamiokande i," Phys. Rev. D, vol. 71, p. 112005, 2005.

[21] M. Apollonio et al., "Limits on neutrino oscillations from the chooz experiment," Physics Letters B, vol. 466, pp. 415 - 430, 1999.

[22] Y. Ashie et al., "Evidence for an oscillatory signature in atmospheric neutrino oscillations," Phys. Rev. Lett., vol. 93, p. 101801, 2004.

[23] K. Eitel, "Direct neutrino mass experiments," Nuclear Physics B - Proceedings Supplements, vol. 143, pp. 197 - 204, 2005.

[24] S. Hannestad, "Cosmological limit on the neutrino mass," Phys. Rev. D, vol. 66, p. 125011, 2002. 
[25] D. N. Spergel et al., "First-year wilkinson microwave anisotropy probe (wmap) observations: Determination of cosmological parameters," The Astrophysical Journal Supplement Series, vol. 148, p. 175, 2003.

[26] S. F. King, "Neutrino mass," Contemporary Physics, vol. 48, pp. 195-211, 2007.

[27] M. Goeppert-Mayer, "Double beta-disintegration," Phys. Rev., vol. 48, pp. 512516, 1935.

[28] E. Majorana, "Teoria simmetrica dellelettrone e del positrone," Il Nuovo Cimento (1924-1942), vol. 14, pp. 171-184, 1937.

[29] G. Racah, "Sulla simmetria tra particelle e antiparticelle," Il Nuovo Cimento (1924-1942), vol. 14, pp. 322-328, 1937.

[30] W. H. Furry, "On transition probabilities in double beta-disintegration," Phys. Rev., vol. 56, pp. 1184-1193, 1939.

[31] H. Primakoff, "Angular correlation of electrons in double beta-decay," Physical Review, vol: 85, pp. 888-890, 1952.

[32] H. V. Klapdor-Kleingrothaus and I. V. Krivosheina, "The Evidence for the Observation of $0 \nu \beta \beta$ Decay: the Identification of $0 \nu \beta \beta$ Events from the Full Spectra," Modern Physics Letters A, vol. 21, pp. 1547-1566, 2006.

[33] F. T. Avignone, S. R. Elliott, and J. Engel, "Double beta decay, majorana neutrinos, and neutrino mass," Rev. Mod. Phys., vol. 80, pp. 481-516, 2008.

[34] S. R. Elliott and P. Vogel, "Double beta decay," Annual Review of Nuclear and Particle Science, vol. 52, pp. 115-151, 2002.

[35] F. Šimkovic, "Neutrinoless double-beta decay and related topics," Physics of Particles and Nuclei, vol. 42, pp. 598-612, 2011.

[36] S. Agostinelli et al., "Geant4 - a simulation toolkit," Nuclear Instruments and Methods in Physics Research Section A: Accelerators, Spectrometers, Detectors and Associated Equipment, vol. 506, pp. 250 - 303, 2003.

[37] S. Biagi, "Monte carlo simulation of electron drift and diffusion in counting gases under the influence of electric and magnetic fields," Nuclear Instruments and Methods in Physics Research Section A: Accelerators, Spectrometers, Detectors and Associated Equipment, vol. 421, pp. 234 - 240, 1999. 
[38] R. Veenhof. (2010, Sept.) Garfield - simulation of gaseous detectors. http://garfield.web.cern.ch/garfield

[39] C. Delaere. (2012, Apr.) TMultiLayerPerceptron. http://root.cern.ch/root/ html/TMultiLayerPerceptron.html

[40] N. Ackerman et al., "Observation of two-neutrino double-beta decay in ${ }^{136} \mathrm{Xe}$ with the exo-200 detector," Phys. Rev. Lett., vol. 107, p. 212501, 2011.

[41] The KamLAND-Zen Collaboration, "Measurement of the double- $\beta$ decay half-life of ${ }^{136}$ Xe with the KamLAND-Zen experiment," Phys. Rev., vol. C85, p. 045504, 2012.

[42] D. Nygren, "High-pressure xenon gas electroluminescent tpc for 0--decay search," Nuclear Instruments and Methods in Physics Research Section A: Accelerators, Spectrometers, Detectors and Associated Equipment, vol. 603, pp. 337 $-348,2009$.

[43] A. Breskin, "Csi uv photocathodes: history and mystery," Nuclear Instruments and Methods in Physics Research Section A: Accelerators, Spectrometers, Detectors and Associated Equipment, vol. 371, pp. 116 - 136, 1996.

[44] R. Platzman, "Total ionization in gases by high-energy particles: An appraisal of our understanding," The International Journal of Applied Radiation and Isotopes, vol. 10, pp. $116-127,1961$.

[45] L. M. P. Fernandes et al., "Primary and secondary scintillation measurements in a xenon gas proportional scintillation counter," Journal of Instrumentation, vol. 5, p. P09006, 2010.

[46] M. Mimura et al., "Average numbers of scintillation photons and electrons produced by an alpha particle in high-density xenon gas," Japanese Journal of Applied Physics, vol. 48, p. 076501, 2009.

[47] M. Suzuki and S. Kubota, "Mechanism of proportional scintillation in argon, krypton and xenon," Nuclear Instruments and Methods, vol. 164, pp. 197 - 199, 1979.

[48] T. D. Bonifield et al., "Time resolved spectroscopy of xenon excimers excited by synchrotron radiation," The Journal of Chemical Physics, vol. 72, pp. 2914-2924, 1980 . 
[49] A. Peisert and F. Sauli, "Drift and diffusion of electrons in gases: A compilation (with an introduction to the use of computing programs)," 1984, CERN-84-08.

[50] V. M. Atrazhev, I. V. Chernysheva, and T. Doke, "Transport properties of electrons in gaseous xenon," Japanese Journal of Applied Physics, vol. 41, pp. 1572 $1578,2002$.

[51] C. M. B. Monteiro et al., "Secondary scintillation yield in pure xenon," Journal of Instrumentation, vol. 2, p. P05001, 2007.

[52] F. Favata, A. Smith, M. Bavdaz, and T. Z. Kowalski, "Light yield as a function of gas pressure and electric field in gas scintillation proportional counters," Nuclear Instruments and Methods in Physics Research Section A: Accelerators, Spectrometers, Detectors and Associated Equipment, vol. 294, pp. 595 - 601, 1990.

[53] A. Fonseca et al., "Study of secondary scintillation in xenon vapour," Nuclear Science Symposium Conference Record, 2004 IEEE, pp. 572 - 576, 2004.

[54] A.J.P.L. and Policarpo, "Ionizations scintillation detectors," Nuclear Instruments and Methods in Physics Research, vol. 196, pp. 53 - 62, 1982.

[55] Geant4 Collaboration, Geant4 User's Guide for Application Developers, December 2011, version: geant4 9.5.0.

[56] P. Truscott. (1999) Treatment of radioactive decay in geant4. http: //www.space.qinetiq.com/geant4/rdm.html

[57] T. W. Burrows, "The evaluated nuclear structure data file: Philosophy, content, and uses," Nuclear Instruments and Methods in Physics Research Section A: Accelerators, Spectrometers, Detectors and Associated Equipment, vol. 286, pp. 595-600, 1990.

[58] F. Boehm and P. Vogel, Physics of Massive Neutrinos. Cambridge: Cambridge University Press, 1992.

[59] D. Lide, Ed., CRC Handbook of Chemistry and Physics, 87th ed. Boca Raton, FL: CRC Press, 2007.

[60] M. Yamashita et al., "Scintillation response of liquid xe surrounded by ptfe reflector for gamma rays," Nuclear Instruments and Methods in Physics Research Section A: Accelerators, Spectrometers, Detectors and Associated Equipment, vol. 535, pp. $692-698,2004$. 
[61] J. W. Fleming, Handbook of optical materials, M. Weber, Ed. Boca Raton: CRC Press, 2003, p. 237.

[62] Geant4 Collaboration, Physics Reference Manual, December 2010, version: geant4 9.4.

[63] G. S. Hurst, L. B. O'Kelly, E. B. Wagner, and J. A. Stockdale, "Time-of-Flight Investigations of Electron Transport in Gases," Journal of Chemical Physics, vol. 39 , pp. 1341-1345, 1963.

[64] J. L. Pack, R. E. Voshall, and A. V. Phelps, "Drift velocities of slow electrons in krypton, xenon, deuterium, carbon monoxide, carbon dioxide, water vapor, nitrous oxide, and ammonia," Phys. Rev., vol. 127, pp. 2084-2089, 1962.

[65] P. Rachinhas et al., "Photoelectron collection efficiency in ch4 and xe-ch4 mixtures," Nuclear Science Symposium Conference Record, 2005 IEEE, vol. 3, pp. $1271-1274,2005$.

[66] H. L. Brooks et al., "Electron drift velocities in xenon," Journal of Physics D: Applied Physics, vol. 15, p. L51, 1982.

[67] European Commission, "Radiological protection principles concerning the natural radioactivity of building materials, radiation protection report rp-112," http://ec.europa.eu/energy/nuclear/radiation_protection/publications_en.htm, 1999.

[68] J. H. Hubbell and S. M. Seltzer. (2004, July) Tables of x-ray mass attenuation coefficients and mass energy-absorption coefficients from $1 \mathrm{kev}$ to $20 \mathrm{mev}$ for elements $\mathrm{z}=1$ to 92 and 48 additional substances of dosimetric interest. Retrieved January 2012. http://www.nist.gov/pml/data/xraycoef/index.cfm

[69] National Nuclear Data Center (NNDC), Brookhaven National Laboratory. Chart of nuclides. Retrieved January 2012. http://www.nndc.bnl.gov/

[70] Argonne National Laboratory (ANL). (2005) Natural decay series: Uranium, radium, and thorium. Retrieved January 2012. www.ead.anl.gov/pub/doc/ natural-decay-series.pdf

[71] Q. He and K. McDonald: (2009, May) Muon rate in the boone tpc. Retrieved March 2012. http://microboone-docdb.fnal.gov/cgi-bin/ShowDocument?docid= 430 
[72] S. Haykin, Neural networks: a comprehensive foundation. New York: Macmillan, 1994.

[73] I. Aleksander and H. Morton, An introduction to neural computing. London: Chapman and Hall, 1990.

[74] C. Bishop, Neural networks for pattern recognition. Oxford: Clarendon Press, 1995.

[75] J. Schwindling. (2000; May) Mlpfit: a tool for multi-layer perceptrons. http://schwind.home.cern.ch/schwind/MLPfit.html 